Universidad Nacional de La Plata

Departamento de Economía

\title{
Inestabilidad \\ macroeconómica en \\ Argentina: crisis \\ financieras e inflación
}

Laura D 'Amato

Doctorado en Economía

Mayo 2009 


\section{Índice general}

$\begin{array}{lll}\text { I Introducción } & \mathbf{1}\end{array}$

$\begin{array}{llr}\text { II Desarrollo } & \mathbf{8}\end{array}$

1. Determinantes de las crisis bancarias en las economías emergentes: $\begin{array}{lr}\text { ¿Fundamentos o profecías autocumplidas? } & \\ & \end{array}$

1.1. Los pánicos bancarios en la literatura . . . . . . . . . . . . . . . 11

1.2. Principales características de la crisis argentina de 2001-2002 . . . . . 12

1.2.1. Una primera revisión de los datos . . . . . . . . . . . . . 14

1.3. Resultados empíricos . . . . . . . . . . . . . . . . 17

1.3.1. Nuestra metodología . . . . . . . . . . . . . . . . . 17

1.3.2. Las técnicas de estimación . . . . . . . . . . . . . . . 18

1.3.3. El modelo empírico . . . . . . . . . . . . . . . . . 18

1.3.4. Resultados econométricos . . . . . . . . . . . . . 22

1.4. Conclusiones . . . . . . . . . . . . . . . . . 27

1.5. Apéndice A. Correlaciones de Spearman . . . . . . . . . . . . . . 30

2. Dinámica inflacionaria, persistencia y cambio de régimen 35

2.1. Motivación . . . . . . . . . . . . . . . 35

2.2. El fenómeno de la persistencia inflacionaria en la literatura: Formación de precios y persistencia de la inflación . . . . . . . . . . . 37

2.3. Algunos rasgos de la dinámica inflacionaria en Argentina . . . . . . . . 46

2.4. Análisis descriptivo . . . . . . . . . . . . . . . . . 48

2.5. Identificando quiebres en la tasa media de inflación . . . . . . . . . 50

2.5.1. Análisis recursivo . . . . . . . . . . . . . . . 51

2.5.2. Test de Bai Perron . . . . . . . . . . . . . . . . 53

2.6. Persistencia inflacionaria . . . . . . . . . . . . . . 55

2.6.1. Las medidas convencionales de persistencia inflacionaria . . . . 55

2.6.2. Persistencia y cambios en la media de la inflación en Argentina 56

2.6.3. Un análisis detallado del período de baja inflación . . . . . . . 61

2.7. Conclusiones . . . . . . . . . . . . . . . . . . . 65

\footnotetext{
${ }^{1}$ Agradecemos los valiosos comentarios de Federico Sturzenegger, Walter Sosa Escudero, Lorena Garegnani y Cristián Castro y las de participantes en los seminarios y congresos en que anteriores versiones del trabajo fueron presentadas.
} 
3. Pronóstico de inflación utilizando múltiples predictores: modelos causales y modelos a-teóricos

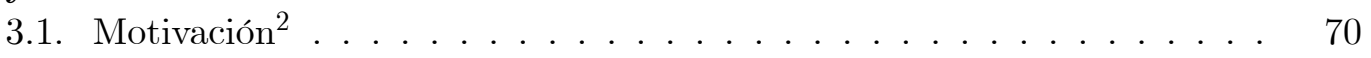

3.2. Los modelos de pronóstico en la literatura . . . . . . . . . . . . 72

3.2.1. Pooling de pronósticos . . . . . . . . . . . . . . 72

3.2.2. Dynamic Factor Models . . . . . . . . . . . . . . . . 74

3.3. Los resultados empíricos . . . . . . . . . . . . . . . . 75

3.3.1. La capacidad predictiva de los modelos estimados . . . . . . . 76

3.3.2. Comparando la capacidad predictiva de modelos alternativos . 77

3.4. Conclusiones . . . . . . . . . . . . . . . . . 81

3.5. Apéndice A: El método de componentes principales . . . . . . . . . . 83

3.6. Apéndice B: Detalle de las series del ciclo utilizadas para el cálculo de factores ........................ 85

3.7. Apéndice C: Modelos de pronóstico . . . . . . . . . . . . . . . 89

3.8. Apéndice D: Tests para comparar capacidad predictiva . . . . . . . . 90

\section{Conclusiones Generales}

${ }^{2}$ Agradecemos las valiosas sugerencias de Hildegart Ahumada, Carlos Capistrán, Bárbara Rossi y Kenneth West, asi como también los comentarios recibidos en el Seminario en el Banco Central de Chile, en octubre de 2008 y en la XLII Reunión de la AAEP en noviembre de 2008. 


\section{Agradecimientos}

Agradezco muy especialmente a Fernando Navajas por sus valiosos aportes al desarrollo de este trabajo y su invalorable respaldo durante todo el tiempo que trabajé bajo su dirección. También le agradezco a Lorena Garegnani sus valiosos comentarios y apoyo en todo este tiempo y a Tamara Burdisso y Verónica Cohen Sabban por haberme acompañado y ayudado en este proceso. También al director del Programa de Doctorado de la UNLP, Alberto Porto y a Walter Sosa Escudero por sus aportes y comentarios a versiones preliminares de mi trabajo al igual que a mis compañeros de doctorado y participantes en los seminarios del Departamento de Economía de la UNLP. Del mismo modo, agradezco a Hernán Lacunza y al BCRA por facilitar mi cumplimiento con el Programa de Doctorado. Mi agradecimiento también a Emilio Blanco por su ayuda en la edición de esta tesis. Finalmente, les agradezco a Jorge, Juan Pablo y Martín, que, como siempre, estuvieron cerca, acompañándome generosamente. 


\section{Parte I}

\section{Introducción}


Esta tesis se concentra en el estudio de dos aspectos de la economía argentina que han sido relevantes para su desempeño macroeconómico: la inestabilidad financiera y la dinámica inflacionaria.

El capítulo I está enfocado al estudio de los determinantes de las crisis financieras. La teoría económica provee varias explicaciones para estos eventos, que en la experiencia argentina han sido muy disruptivos en términos de actividad y crecimiento económico. En el trabajo pionero de Diamond y Dybvig (1983) las crisis bancarias son profecías autocumplidas que pueden generanse por errores de percepción de los agentes económicos respecto de las necesidades de liquidez de otros agentes, que eventualmente llevan a un mal equilibrio en el que se produce una corrida bancaria. En una visión alternativa, las crisis financieras pueden verse como un ejercicio de disciplina del mercado a través del cual los depositantes castigan el excesivo riesgo asumido por las instituciones financieras, como en Gorton (1985) o Chari y Jagannathan o Jacklin y Bhattacharya (1988), entre otros. Finalmente, las crisis financieras pueden ser un fenómeno de equilibrio, es decir, la respuesta de los agentes económicos a un deterioro de los fundamentos macroeconómicos, como en Wallace (1988, 1990) y Hellwig (1994). En esa dirección, Allen y Gale (1998) desarrollaron un modelo en el cual las corridas bancarias son la respuesta natural de los agentes económicos a un aumento del riesgo agregado debido a una reducción del valor de los activos de las empresas a causa de, por ejemplo, una caída en la actividad económica. Ennis (2002) ha mostrado más recientemente que las corridas bancarias inducidas por profecías autocumplidas también pueden estar correlacionadas positivamente con malos fundamentos en un modelo con equilibrios múltiples.

En las Economías de los Mercados Emergentes (EME), estos episodios suelen desarrollarse como una combinación de crisis monetaria y bancaria y son, con frecuencia, muy costosas en términos de crecimiento económico. ${ }^{3}$ En la segunda mitad de la década de 1990, una serie de crisis financieras golpeó a las EME: la crisis mexicana de 1995, la asiática de 1997, el default ruso de 1998, y más recientemente, la crisis argentina de 2001-2002. Estos enventos renovaron el interés en el tema y muchos afirmaron que se trataba de un nuevo tipo de fenómeno. Sin embargo, Chang y Velasco (1998) subrayan las características comunes de estos episodios con crisis anteriores: reformas económicas orientadas al mercado; liberalización comercial y financiera; desregulación y privatización de empresas públicas. También enfatizan que la fragilidad financiera, debida a una regulación y a una supervisión bancarias inadecuadas, fue una debilidad importante que casi todas estas experiencias tuvieron en común.

Indagar sobre los determinantes de las crisis financieras puede contribuir al diseño y mejora de políticas y regulaciones que apunten a reducir la probabilidad de ocurrencia

\footnotetext{
${ }^{3}$ Véase Kaminsky y Reinhart (1999).
} 
de este tipo de eventos. Por sus peculiaridades, la crisis bancaria argentina de 2001-2002 constituye una oportunidad única para poner a prueba las tres hipótesis propuestas en la literatura sobre las causas de dichos eventos. Al tiempo que comparte algunas características comunes con los episodios anteriores en las EME por el hecho de que desde 1991, el país entró en un proceso de reformas estructurales de mercado y de liberalización financiera, el caso argentino difiere de otras experiencias de EME, en que su sistema bancario era, aparentemente, menos vulnerable a las crisis financieras, debido a la profunda reforma financiera que se implementó en la década de 1990, acercando los estándares de regulación y supervisión a las normas de Basilea.

Desarrollamos en el primer capítulo de esta tesis una metodología que explota la riqueza de un conjunto de datos de panel sobre cambios diarios en los depósitos de bancos individuales, y nos permite deslindar las causas comunes de las individuales en la variación de los depósitos. La dinámica de los depósitos brinda información muy valiosa sobre la forma en que los agentes económicos reaccionaron ante la información relevante que recibían sobre los fundamentos macroeconómicos, así como las respuestas de política y de mercado a estas noticias. La inclusión de fundamentos microeconómicos de los bancos y de fundamentos macroeconómicos nos permiten identificar los determinantes de la conducta de los depositantes.

Los Capítulos II y III se enfocan en el estudio de la dinámica inflacionaria, su relación con los regímenes monetarios y su pronóstico. La inflación es sin duda una variable relevante tanto para la formulación de la política monetaria como para las decisiones de los agentes privados. Argentina experimentó durante largos años inflación persistentemente elevada. Como enfatizan Stock y Watson (2005), la evidencia empírica internacional reciente sugiere que con el descenso de la inflación como un fenómeno extendido en la economía mundial, la dinámica conjunta de la inflación y sus potenciales predictores, como el dinero o alguna medida de la utilización el de los recursos, ha cambiado y la inflación se ha tornado más impredecible. Como también señalan Stock y Watson, los modelos univariados suelen superar en capacidad de pronóstico a los modelos basados en distintas teorías de la inflación y el desempeño de estos últimos se ha deteriorando con el descenso de la inflación.

En el caso de Argentina, evidencia reciente (Basco et al., 2006), indica que es posible encontrar dinámicas de la inflación bastante diferenciadas entre el período de inflación elevada entre 1980 y 1989 y, luego del episodio hiperinflacionario, un período que puede considerarse de inflación baja, entre 1991 y la actualidad. Si bien el cambio de régimen monetario que implicó el abandono del régimen de convertibilidad sugiere la presencia de un cambio estructural a partir de enero de 2002, desde un punto de vista estadístico sólo es posible identificar un período que puede considerarse como atípico entre enero de 2002 y comienzos de 2003. 
Por otro lado, la evidencia empírica proveniente de estimaciones de curvas de Phillips (ver D'Amato y Garegnani, 2008 y, Elosegui y otros, 2007 para Argentina), muestra una relación algo débil entre la brecha del producto y la tasa de inflación durante el período de inflación baja. También el análisis de la dinámica inflacionaria mediante el uso de modelos VAR (Basco et al., op. cit.) indica que en los años recientes, la dinámica de la inflación parece ser más exógena a variables como el dinero, la tasa de interés y el producto que durante los años de inflación elevada y por lo tanto más difícil de predecir utilizando modelos multivariados.

Los determinantes de largo plazo de la inflación han sido ampliamente estudiados en la teoría monetaria y existe acuerdo acerca de que el crecimiento monetario y la inflación están altamente correlacionados en el largo plazo y que esa relación no es única sino dependiente del régimen monetario vigente. En los años recientes la evidencia empírica ha puesto en evidencia que la relación de largo plazo entre crecimiento monetario no es única, y que esta se debilita con el descenso de la tasa de inflación. En suma, si bien los determinantes de la inflación no son únicos, un elevado crecimiento monetario de largo plazo parece estar asociado a altas tasas de inflación. Las motivos de las altas tasas de expansión monetaria son al menos dos: el financiamiento monetaria de los desequilibrios fiscales o el persistente intento de explotar el trade-off entre desempleo e inflación con fines expansivos.

Vinculado a lo anterior, el fenómeno de la persistencia inflacionaria, definida como la velocidad con que la inflación se aproxima a su valor de largo plazo luego de un shock, también parece haber cambiado con el descenso de la inflación. Este fenómeno ha sido ampliamente estudiado en los años recientes para las economías industriales, pero la evidencia para países en desarrollo es bastante más limitada. Es también relevante para la modelación e implementación de la política monetaria determinar el grado de persistencia de la inflación, ya que la capacidad del banco central para estabilizarla en torno a algún valor de largo plazo depende en gran medida del grado de rigidez nominal que exista en la economía. La evidencia internacional reciente sugiere que una vez que se toma en cuenta que el valor de largo plazo de la inflación no es constante, el grado de persistencia se reduce. También hay cierta evidencia de que la persistencia ha sido más elevada en períodos de alta inflación.

El Capítulo II se enfoca al estudio de los cambios en la dinámica inflacionaria y su relación con los cambios de régimen monetario a lo largo de un prolongado período. Utilizando un modelo neo-keynesiano estándar, se realiza un ejercicio de simulación para evaluar la incidencia de distintos niveles de persistencia en el impacto que puede tener la política monetaria sobre la actividad económica.Se utilizan el análisis recursivo y tests de quiebre estuctural para identificar cambios tanto en la media como en el componente autorregresivo de la inflación. También se calculan medidas de presistencia 
que controlan por esos cambios.

En el Capítulo III se evalúan distintos modelos de pronóstico de inflación, desde modelos univariados hasta modelos causales como la curva de Phillips y modelos monetarios. También se trabaja con modelos de pronóstico basados en el uso de factores, como medidas resumidas de la variabilidad conjunta de un gran número de series del ciclo económico, como proponen, entre otros, Stock y Watson (1999). Se compara la capacidad predictiva de los modelos individuales utilizando un conjunto de tests estadísticos. Finalmente se evalúa la capacidad de un pooling de pronóstico de superar a los modelos individuales. 


\section{Bibliografía}

[1] Allen, F y D. Gale, "Optimal Financial Crisis". The Journal of Finance. Vol. LIII, n. ${ }^{\circ}$ 4. Agosto 1998.

[2] Basco E., L. D 'Amato y L. Garegnani, (2006), "Understanding the money and prices relationship under low and high inflation: Argentina 1970-2006", Documento de Trabajo 2006/18, BCRA.

[3] Chang, R. y A. Velasco (1998), "The Asian Liquidity Crisis", Working Paper Nro. 6796, NBER.

[4] Chari, V., y R. Jagannathan (1988), "Banking Panics, Information and Rational Expectations Equilibrium", Journal of Finance 43, 749-760.

[5] D 'Amato, L. y L. Garegnani (2006), "The Short -run Dynamics of Inflation:Estimating a "Hybrid New Keynesian Phillips Curve" for Argentina", Monetaria, Volumen X, CEMLA.

[6] Diamond, D. y P. Dyvbig (1983), "Bank Runs, Deposit Insurance and Liquidity", Journal of Political Economics 91, 401-419.

[7] Elosegui P., G Escudé, L. Garegnani y J.M. Sotes Paladino (2007), "El Modelo Económico Pequeño del BCRA", Documento de Trabajo 2007/18, BCRA.

[8] Ennis, H. (2002), "Economic Fundamentals and Bank Runs", Federal Reserve Bank of Richmond, mimeo.

[9] Fuhrer, J.. y G. Moore (1995a), "Inflation Persistence", Quarterly Journal of Economics 110 (1): 127-159.

(1995b), "Monetary Policy Trade-Offs and the Correlation Between Nominal Interest Rates and Real Output", The American Economic Review 85 (marzo): 219-239.

(2006), "Intrinsec and Inhereted Inflation Persistence", International Journal of Central Banking, septiembre, Vol. $2 \mathrm{~N}^{\circ} 3$. 
[10] Gorton, G.(1985), "Banks Suspension of Convertibility", Journal of Monetary Economics, 15: 177-93.

[11] Hellwig, M. (1994), "Liquidity provisioning, banking, and the allocation of interest rate risk". European Economic Review 38, 1363-1389. .

[12] Jacklin, Ch .J. y S. Bhattacharya, (1988), "Distinguishing Panics and Informationbased bank runs: Welfare and Policy implications". Journal of Political Economy 96, 586-592.

[13] Kaminsky, G. y C. Reinhart (1999), "The Twin Crises: The Causes of Banking and Balance of Payments Problems", The American Economic Review, junio, 473-500.

[14] Stock, J. y M. Watson (2005), "Why has U.S. Inflation become harder to forecast?", WP N¹2324,NBER.

[15] Wallace, N. (1988), "Another attempt to explain an illiquid banking system: The diamond and Dybig model with sequential service taken seriously". Federal Bank of Minneapolis Quarterly Review 12 (4):3-16.

[16] Wallace, N. (1990), " A banking model in which suspension is best", Federal Reserve Bank of Minneapolis Quarterly Review, 14. 


\section{Parte II}

\section{Desarrollo}




\section{Capítulo 1}

\section{Determinantes de las crisis bancarias en las economías emergentes: ¿Fundamentos o profecías autocumplidas? ${ }^{1}$}

La bibliografía teórica moderna sobre crisis bancarias ofrece tres explicaciones básicas al pánico bancario: (i) son el producto de un mal equilibrio, es decir, hechos aleatorios provocados por percepciones incorrectas de los agentes sobre sus necesidades de liquidez; (ii) son un fenómeno de equilibrio, es decir, la respuesta de los agentes económicos a un deterioro de los fundamentos macroeconómicos; (iii) son un ejercicio de disciplina del mercado a través del cual los depositantes castigan el excesivo riesgo asumido por las instituciones financieras.

En las Economías de los Mercados Emergentes (EME), estos hechos suelen desarrollarse como "crisis gemelas", es decir una combinación de crisis monetaria y bancaria y son, con frecuencia, muy costosas en términos de crecimiento económico. ${ }^{2}$ En la segunda mitad de la década de 1990, una serie de crisis financieras golpeó a las EME: la crisis mexicana de 1995, la asiática de 1997, el default ruso de 1998, y más recientemente, la crisis argentina de 2001-2002. Esto renovó el interés en el tema y muchos afirmaron que se trataba de un nuevo tipo de fenómeno. Sin embargo, Chang y Velasco (1998) subrayan las características comunes de estos episodios con crisis anteriores: reformas económicas orientadas al mercado; liberalización comercial y financiera; desregulación

\footnotetext{
${ }^{1}$ Agradecemos los valiosos comentarios de Federico Sturzenegger, Walter Sosa Escudero, Lorena Garegnani y Cristián Castro y las de participantes en los seminarios y congresos en que anteriores versiones del trabajo fueron presentadas.

${ }^{2}$ Véase Kaminsky y Reinhart (1999).
} 
y privatización de empresas públicas. También enfatizan que la fragilidad financiera, debida a una regulación y a una supervisión bancarias inadecuadas, constituyó una importante debilidad que casi todas estas experiencias tienen en común. ${ }^{3}$

Más recientemente, Allen y Gale (2001) señalan que la creación previa de burbujas de activos y de deuda pendiente, debidas al excesivo riesgo asumido por los intermediarios financieros, así como la falta de una política monetaria estable, que hace que el time-path de los créditos sea impredecible, parecen ser características comunes en los países proclives a las crisis financieras.

Aquí desarrollamos una metodología empírica para poner a prueba las tres hipótesis descriptas anteriormente, usando datos microeconómicos sobre el comportamiento de los depósitos en bancos individuales, y la aplicamos al estudio de la crisis bancaria y monetaria argentina de 2001-2002.

La crisis bancaria argentina de 2001-2002 brinda una oportunidad única para poner a prueba las tres hipótesis mencionadas. Por un lado, comparte algunas características comunes con los episodios anteriores en las EME: desde 1991, el país entró en un proceso de reformas estructurales de mercado y de liberalización financiera. En 1991 se introdujo un régimen de convertibilidad, que estabilizó la economía y permitió casi diez años de crecimiento sostenido y alta inversión, alimentados por el flujo de capitales y una gran expansión del crédito. Por otro lado, el caso argentino difiere de otras experiencias de EME, en que su sistema bancario era, aparentemente, menos vulnerable a las crisis financieras. Durante la década de 1990 se implementó una profunda reforma financiera que impuso regulaciones y supervisión de acuerdo con las normas de Basilea. Sin embargo, había causas ocultas de fragilidad financiera: (i) la dolarización de los balances bancarios imponía un alto riesgo en caso de que fuera necesaria una reacomodación del tipo de cambio y (ii) la gran exposición de los bancos al riesgo soberano.

La metodología que desarrollamos aquí explota la riqueza de un conjunto de datos de panel sobre cambios diarios en los depósitos de bancos individuales, y nos permite deslindar las causas comunes de las individuales en la variación de los depósitos. La idea es que la dinámica de los depósitos puede brindar una rica información sobre la forma en que los agentes económicos reaccionaron ante la información relevante que recibían sobre los fundamentos macroeconómicos, así como las respuestas de política y de mercado a estas noticias. Los fundamentos microeconómicos de los bancos también se incluyen como determinantes potenciales de la conducta del depositante. Queremos identificar los factores relevantes que desencadenaron la crisis bancaria y monetaria: hasta qué punto la corrida fue causada por la percepción de los depositantes de un aumento del riesgo agregado en lugar de ser una corrida de bancos particulares. Si ni

\footnotetext{
${ }^{3}$ Véase Díaz Alejandro (1985) para una descripción ilustrativa y detallada del caso chileno.
} 
los fundamentos macroeconómicos, ni los fundamentos microeconómicos específicos de los bancos ayudan a explicar esta dinámica, ¿se podría concluir que fue un fenómeno de "mancha solar"?

Este capítulo está organizado de la siguiente forma: en la sección 2, revisamos la literatura teórica; en la sección 3, describimos los principales rasgos de la crisis argentina de 2001, en la sección 4 presentamos el análisis empírico y, finalmente, en la sección 5 presentamos las conclusiones.

\subsection{Los pánicos bancarios en la literatura}

Como es bien conocido, se ha dedicado una cantidad considerable de investigación teórica y empírica a explicar el fenómeno de los pánicos bancarios. ${ }^{5}$ La mayoría de los desarrollos teóricos en este campo se fundan en el trabajo pionero de Diamond y Dybvig (1983). Estos autores modelan las crisis bancarias como profecías autocumplidas, en las cuales las necesidades de liquidez individual se alimentan de una serie de errores de percepción de los agentes económicos respecto de las necesidades de otros agentes, que eventualmente llevan a un mal equilibrio en el cual se genera una corrida bancaria.

Una explicación teórica alternativa para el pánico bancario es que este se genera por un aumento del riesgo agregado. Wallace $(1988,1990)$, Jacklin y Bhattacharya (1988), Chari y Jagannathan (1998), y Hellwig (1994) desarrollaron modelos en esta línea. Recientemente, Chang y Velasco (2000, 2001), y Kawamura (2002) extendieron este argumento al caso de una pequeña economía abierta que enfrenta "iliquidez financiera" como una posible explicación para las recientes crisis internacionales como las de Asia en 1997 o la de Brasil en 1999.

Allen y Gale (1998) desarrollaron un modelo en el cual las corridas bancarias son la respuesta natural de los agentes económicos a un aumento del riesgo agregado debido a una reducción del valor de los activos de las empresas a causa de, por ejemplo, una caída en la actividad económica. En este sentido, si un indicador importante anticipa una recesión inminente, puede inducir un retiro de depósitos, como respuesta a una caída esperada de los precios de los activos de las firmas, deteriorando la cartera de los bancos. Así el pánico bancario está causado por un problema de solvencia en lugar de uno de liquidez. Un resultado importante es que las corridas bancarias pueden dar lugar a una asingación óptima del riesgo y por lo tanto la no intervención del gobierno podría ser la mejor política.

Ennis (2002) ha mostrado recientemente que las corridas bancarias inducidas por profecías autocumplidas también pueden estar correlacionadas positivamente con mal-

\footnotetext{
${ }^{5}$ Para un buen y detallado tratamiento de los principales desarrollos teóricos en este campo, véase Freixas y Rochet (1987), capítulo 7.
} 
os fundamentos en un modelo con múltiples equilibrios. Expone dos formas a través de las cuales se produce la elección de un equilibrio: un mecanismo de selección en el cual se elige el mejor equilibrio, y un proceso de aprendizaje. Contrario al modelo de Allen y Gale (1998), donde las corridas bancarias son un resultado óptimo, en un contexto de múltiples equilibrios, hay lugar para políticas que impidan que se produzca una corrida.

Nuestra suposición aquí es que, en lugar de ser un fenómeno de "mancha solar", o un pánico bancario debido a un riesgo excesivo asumido por algunas instituciones financieras, y extendido al sistema financiero por efectos de contagio, esta crisis bancaria y monetaria fue provocada por un deterioro de los fundamentos macroeconómicos. Probaremos aquí la relevancia de esta hipótesis en comparación con las otras dos, haciendo uso de un rico conjunto de datos sobre los cambios diarios en los depósitos de bancos individuales.

\subsection{Principales características de la crisis argentina de 2001-2002}

En enero de 2002, Argentina abandonó la Convertibilidad y pasó a un régimen de tipo de cambio de flotación en medio de la crisis económica, política e institucional, probablemente, más profunda experimentada por el país en años. El abandono de la convertibilidad fue solo el último paso de un largo proceso en el que la economía, inmersa en una profunda y prolongada recesión desde la segunda mitad de 1998, perdió gradualmente acceso a los mercados financieros internacionales y sufrió, durante el año 2001, una crisis bancaria que el gobierno trató, sin éxito, de reprimir, imponiendo restricciones al retiro de depósitos, hasta que finalmente declaró el default de su deuda y dejó que el tipo de cambio flotara. Se han ofrecido diferentes explicaciones sobre las raíces de este hecho y su dinámica particular. En general, todas ellas coinciden en ver la crisis como un fenómeno muy complejo, imposible de ser comprendido como generado por una causa única. ${ }^{7}$

Como se señaló anteriormente, el sistema financiero argentino era aparentemente sólido debido a una profunda reforma financiera implementada en la década de 1990. Sin embargo, la crisis de 2001-2002 reveló que había al menos dos riesgos potenciales para el sector financiero que fueron subestimados durante los buenos tiempos de la Convertibilidad.

En primer lugar, el financiamiento del sector privado, principalmente en dólares,

\footnotetext{
${ }^{7}$ Galiani Heymann y Tomassi (2003), Calvo y otros (2003), Perry y Serven (2003); Hausman y Velasco (2002), Powell (2002), Carrera (2002) y Della Paolera y Taylor (2003) ofrecen muy buenas descripciones de los determinantes de esta crisis.
} 
daba fondos a los prestatarios nacionales del sector privado involucrados en actividades no transables. La corrección de un desajuste del tipo de cambio real por una deflación o una devaluación nominal podía dañar seriamente la solvencia de los prestatarios del sector no transable, haciéndolos incapaces de pagar sus deudas con los bancos. Una corrección del desajuste del tipo de cambio real, a través de una deflación o de una devaluación de la moneda, implicaba un problema de solvencia para el sistema financiero, ya que aproximadamente el $65 \%$ del crédito al sector privado nacional no financiero (cuyo ingreso era principalmente en pesos) estaba denominado en dólares. En un sistema financiero altamente dolarizado el banco central estaba limitando en su capacidad de actuar como prestamista de última instancia frente a la eventualidad de una crisis financiera.

La segunda fuente de fragilidad financiera era la exposición de los bancos a la deuda del gobierno, en forma de préstamos o de bonos, dadas las restricciones impuestas por el régimen de convertibilidad al financiamiento monetario del gobierno. La alta exposición de los bancos a la deuda del gobierno también implicaba para el sector financiero un riesgo para la solvencia. ${ }^{9}$

El gráfico 1 muestra la evolución de los depósitos totales del sector privado durante los años 2000 y 2001. Nuestro período de análisis es julio de 2000-noviembre de 2001. Consideramos la caída de depósitos de noviembre de 2000 como el episodio inicial de la crisis, que evolucionó lentamente con alzas y bajas, hasta que se declaró una especie de inconvertibilidad a finales de noviembre de 2001, el llamado "Corralito" ${ }^{10}$ Después de su implementación, la dinámica de los depósitos es muy ruidosa, lo que refleja los efectos de las restricciones a los retiros, la pesificación asimétrica y los persistentes intentos de los ahorristas de preservar el valor de sus activos. No analizamos estas cifras aquí y restringimos nuestro análisis al período antes mencionado.

Identificamos cuatro episodios de retiro de depósitos: El primero comprende el período 08/11/00 - 13/12/00, el segundo 12/02/01-30/03/01, el tercero 04/07/01 $23 / 08 / 01$ y el cuarto, $1 / 10 / 01-30 / 11 / 01$.

Casi todos ellos se desencadenaron a causa de hechos políticos o del fracaso de medidas políticas tendientes a recuperar la confianza, y pueden identificarse fácilmente en el Gráfico 1. En la sección 1.3.1 presentamos un análisis descriptivo de estos episodios.

\footnotetext{
${ }^{9}$ En noviembre de 2001, el $24 \%$ de los activos totales de los bancos correspondían a préstamos al sector público y bonos del gobierno.

${ }^{10}$ A partir de la corrida bancaria del 30 de noviembre de 2001, el gobierno impuso fuertes restricciones al retiro de depósitos, las que se profundizaron durante la crisis.
} 
Gráfico 1: Depósitos totales del sector privado

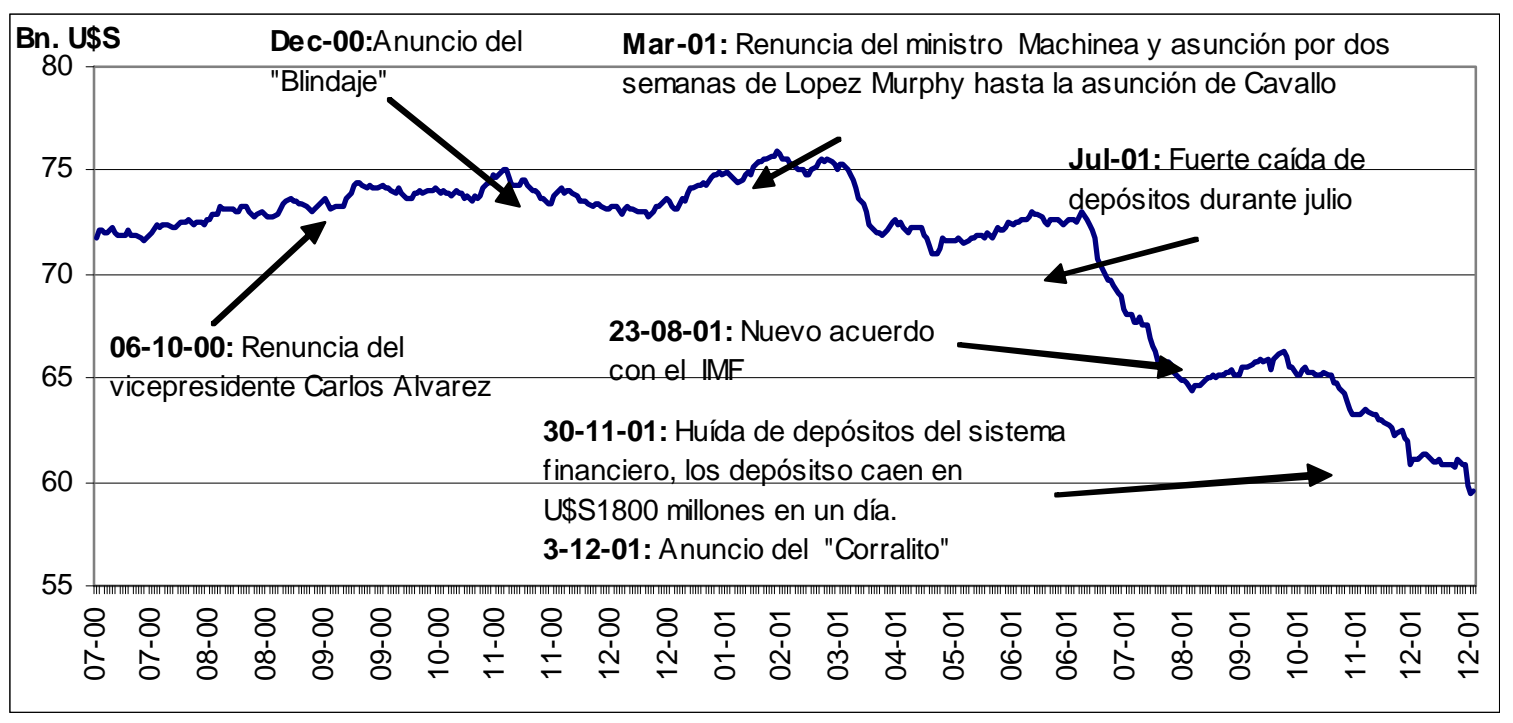

Fuente: BCRA

\subsubsection{Una primera revisión de los datos}

Llevamos a cabo aquí un análisis descriptivo de la dinámica de los distintos grupos de bancos durante los cuatro episodios, después de clasificarlos de acuerdo con dos criterios diferentes: origen del capital y tamaño. ${ }^{12}$ Queremos determinar (i) si esta dinámica cambió en el tiempo a medida que los depositantes recibían nueva información sobre la economía, (ii) si había diferencias relevantes en el desempeño en los grupos de bancos, lo que daría pruebas de una "fuga hacia la calidad" o de "efectos de contagio". Los resultados se presentan en los Gráficos 2 y 3 y las Tablas 1 y 2.

En el primer episodio, del 08/11/00 al 13/12/00, la caída de los depósitos del sector privado fue modesta: $0,67 \%$. Las instituciones financieras de propiedad nacional perdieron más depósitos que las de propiedad extranjera. De acuerdo con el tamaño del banco, los bancos privados más pequeños fueron los que más perdieron. Así, la dinámica de los depósitos durante este primer período da una indicación de un tipo de efecto de "fuga a la calidad".

\footnotetext{
${ }^{12}$ Clasificamos los bancos privados minoristas por tamaño en dos grupos: los cinco más grandes versus el resto. Un segundo criterio de clasificación es el origen del capital, separando a los bancos en tres grupos: de propiedad nacional, extranjera y públicos, grupo este último que incluye los tres más grandes bancos públicos del sistema. Excluimos de la muestra los bancos mayoristas, tanto de propiedad nacional como extranjera, y los que no son bancos, pues estas instituciones financieras no se apoyan en los depósitos como su principal fuente de financiamiento. Los bancos públicos provinciales también están excluidos de la muestra, debido a la mala calidad de las cifras de sus balances.
} 
Durante el segundo episodio, 12/02/01 a 30/03/01, la caída en los depósitos fue más intensa (5,82\%). El grupo de los bancos minoristas privados fue el que más perdió, seguido por los bancos públicos grandes y los cinco más grandes bancos minoristas. Si el criterio es el origen del capital, los bancos extranjeros perdieron más que los nacionales, pero esta diferencia no es estadísticamente significativa. Así, parece que en este período los depositantes huyeron más intensamente de las instituciones financieras más pequeñas, probablemente percibidas como más débiles en comparación con los bancos grandes.

Gráfico 2: Variación depósitos por grupo de bancos según origen del capital

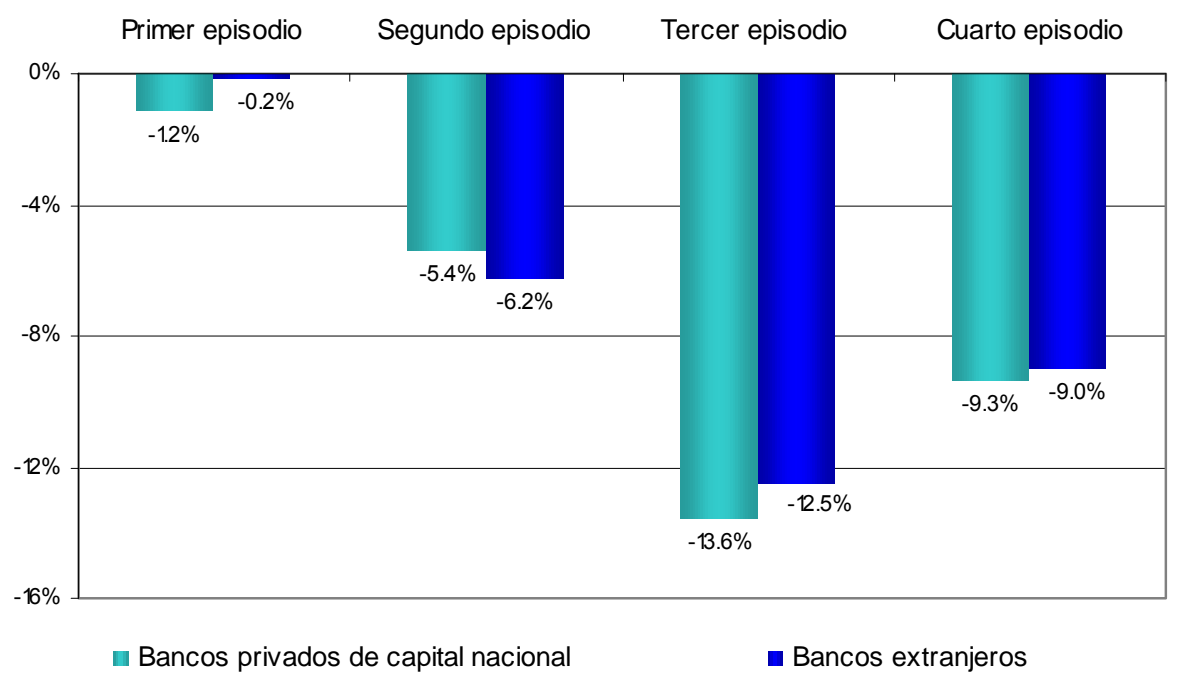

Fuente:BCRA

Tabla 1: Cambio promedio en depósitos sector privado por grupo de bancos según origen del capital

$\begin{array}{lcccc} & \begin{array}{c}\text { desde } 08 / 1 \mathrm{I} / 00 \text { al } \\ 13 / 12 / 00\end{array} & \begin{array}{c}\text { desde } 02 / 12 / 0 \mathrm{I} \text { al } \\ 30 / 03 / 0 \mathrm{I}\end{array} & \begin{array}{c}\text { desde } 04 / 07 / 0 \mathrm{I} \text { al } \\ 23 / 08 / 0 \mathrm{I}\end{array} & \begin{array}{c}\text { desde } 10 / 03 / 0 \mathrm{Ial} \\ 30 / 1 \mathrm{I} / 0 \mathrm{I}\end{array} \\ \text { Bancos de capital nacional } & -0.032 \% & -0.126 \% & -0.271 \% & -0.158 \% \\ \text { Bancos extranjeros } & -0.005 \% & -0.144 \% & -0.250 \% & -0.152 \%\end{array}$

Fuente:BCRA 
Gráfico 3:Variación depósitos por grupo de bancos según tamaño

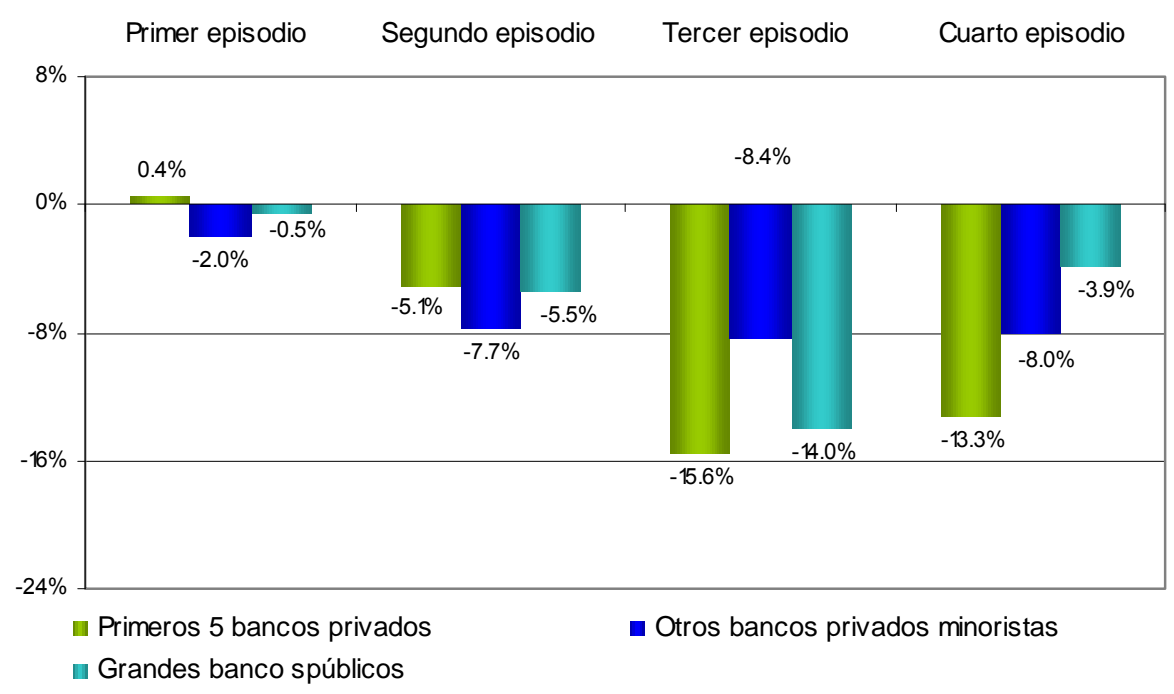

Fuente:BCRA

Tabla 2: Cambio promedio en depósitos sector privado por grupo de banco según

\begin{tabular}{|c|c|c|c|c|}
\hline & $\begin{array}{c}\text { desde } 08 / 11 / 00 \text { al } \\
\qquad 3 / 12 / 00\end{array}$ & $\begin{array}{c}\text { desde } 02 / 12 / 01 \text { al } \\
30 / 03 / 01\end{array}$ & $\begin{array}{c}\text { desde } 04 / 07 / 01 \text { al } \\
23 / 08 / 01\end{array}$ & $\begin{array}{c}\text { desde } 10 / 03 / 0 \text { lal } \\
30 /|1 / 0|\end{array}$ \\
\hline $\begin{array}{l}5 \text { primeros bancos } \\
\text { privados minoristas }\end{array}$ & $0.012 \%$ & $-0.118 \%$ & $-0.312 \%$ & $-0.224 \%$ \\
\hline otros bancos minoristas & $-0.055 \%$ & $-0.179 \%$ & $-0.167 \%$ & $-0.136 \%$ \\
\hline grandes bancos públicos & $-0.015 \%$ & $-0.127 \%$ & $-0.279 \%$ & $-0.067 \%$ \\
\hline todos los bancos & $-0.018 \%$ & $-0.135 \%$ & $-0.260 \%$ & $-0.155 \%$ \\
\hline
\end{tabular}

Fuente:BCRA

En el tercer período, que abarca del 04/07/01 al 23/08/01, el 13,02\% de los depósitos totales del sector privado salieron del sistema financiero, el retiro más intenso de todo el período. Contrario a lo que sucedió en los períodos anteriores, los bancos más grandes sufrieron los retiros más intensos. Los depósitos en los cinco bancos privados más grandes cayeron un $16 \%$, mientras que los depósitos del sector privado en los bancos públicos más grandes disminuyeron en un 14\%. Los depósitos en los bancos privados más pequeños cayeron menos $(8,3 \%)$. Aunque los bancos extranjeros sufrieron una corrida menos intensa, la diferencia respecto de los bancos nacionales 
es insignificante. Así, la dinámica de los depósitos durante este episodio fue completamente diferente de los dos anteriores: la fuga de depósitos fue más intensa y las instituciones financieras más grandes sufrieron una corrida más seria.

Finalmente, en el último período, del 03/10/01 al 30/11/01, en el que los depósitos totales del sector privado cayeron $9 \%$, los cinco bancos más grandes experimentaron una vez más la caída más profunda (13,3\%). Los depósitos en el resto de los bancos privados cayeron un $8 \%$, mientras que los bancos públicos más grandes perdieron solo el $3,9 \%$.

Resumiendo, el análisis descriptivo indica que las diferencias en el origen del capital no fueron relevantes para explicar la actitud de los depositantes hacia los bancos, mientras que el tamaño sí importó. En los dos primeros episodios, las instituciones financieras más pequeñas sufrieron la mayor caída, probablemente porque se las percibía como más débiles que los bancos más grandes. La conducta de los depositantes se revirtió en los últimos dos períodos, cuando los depósitos huyeron más intensamente de los bancos más grandes del sistema. La creciente exposición de los bancos más grandes a la deuda del sector público podría ser una explicación de este comportamiento. En la siguiente sección, usamos el análisis econométrico para tratar de responder a algunas de las preguntas planteadas en la sección 2 .

\subsection{Resultados empíricos}

\subsubsection{Nuestra metodología}

Para evaluar las tres hipótesis planteadas al inicio acerca de las causas de las crisis bancaria construimos un modelo para el cambio de los depósitos de bancos individuales, explicado básicamente por (i) un conjunto de fundamentos macroeconómicos y (ii) un conjunto de fundamentos microeconómicos de los bancos individuales. Nuestro objetivo es determinar en qué medida la crisis fue desencadenada por el deterioro de los fundamentos macroeconómicos, o por el riesgo excesivo asumido por algunas instituciones financieras, o fue simplemente un fenómeno de "mancha solar".

Nos planteamos varias preguntas que pueden ayudar a comprender que llevó a la crisis: (i) ¿la dinámica de los depósitos se explica por movimientos en los fundamentos macroeconómicos?; (ii) ¿fueron importantes los fundamentos de los bancos individuales para explicar el comportamiento de los depósitos, es decir, funcionó la disciplina del mercado en el sentido de que las diferencias en la solidez de los bancos individuales pueden explicar las diferencias en la dinámica de los depósitos?; (iii) ¿hubo alguna evidencia de una fuga a la calidad, es decir, desde los bancos percibidos como más débiles o más riesgosos a aquellos percibidos como más sanos, probablemente por ser 
extranjeros o too big to fail ?

Para intentar responder estas preguntas estimamos un modelo para el cambio diario en los depósitos por banco usando técnicas de datos de panel para evaluar si hay una dinámica común en todos los bancos, es decir, si (i) es verdad; si hay diferencias significativas en la dinámica de los depósitos en los bancos, es decir, si (ii) y (iii) son ciertos. El análisis descriptivo de la sección 3.2 brinda evidencia de diferencias significativas en la dinámica de los depósitos en los cuatro episodios de corrida bancaria y, por lo tanto, las respuestas a estas preguntas podrían diferir según el episodio. Por este motivo, trabajamos con modelos que usan datos diarios para cada período, así como con un modelo que usa datos semanales para toda la muestra (julio de 2000noviembre de 2001), con gran variabilidad, pues incluyen períodos de alzas y bajas en los depósitos.

Dada la naturaleza dinámica de nuestro modelo, utilizamos el método de Arellano y Bond, que es la técnica apropiada para tratar la naturaleza autorregresiva de nuestros datos, para estimar un modelo para los depósitos bancarios durante la crisis.

\subsubsection{Las técnicas de estimación}

Para probar las hipótesis (i) a (iii), utilizamos estimadores de efectos fijos así como de efectos aleatorios para evaluar la presencia de efectos individuales y también temporales.

Luego, dado el carácter dinámico del modelo, implementamos el método de Arellano y Bond, que el es adecuado para tratar algunas características particulares de nuestro modelo: (i) contiene efectos individuales; y (ii) incluye una variable que depende del rezago. Cuando hay efectos individuales inobservables, la variable dependiente se correlaciona con el término de error y eso hace que el estimador OLS sea sesgado e inconsistente, aunque el término de error no se correlacione serialmente. Arellano y Bond sugieren diferenciar primero el modelo para deshacerse de los efectos individuales y usar $y_{i t-1}$ como instrumento.

Sin embargo, este método lleva a estimadores consistentes, pero no eficientes. Dadas las características particulares de nuestro panel (tiene casi el mismo tamaño en ambas dimensiones, $\mathrm{n}$ individuos y $\mathrm{T}$ tiempo, y ambas dimensiones no son "ni grandes ni pequeñas"), el estimador de los efectos fijos no necesariamente es inconsistente. Por esta razón, presentamos los dos estimadores.

\subsubsection{El modelo empírico}

La ecuación 1 describe el modelo estimado 


$$
\begin{aligned}
\Delta \text { dep }_{i t}= & \alpha+\sum_{h=1}^{H} \beta_{h} \Delta d e p_{i t-h}+\sum_{k=1}^{K} \sum_{l=0}^{L} \delta_{k l} X_{i t-l}^{k} \\
& +\sum_{m=1}^{M} \sum_{p=0}^{P} \varepsilon_{m p} W_{t-p}+\sum_{r=1}^{R} \sum_{s=0}^{S} \lambda_{r s} Z_{t-s}^{r}+i_{i t} \\
\text { para } i= & 1, \ldots N \text { y } t=1, \ldots . T
\end{aligned}
$$

La estructura del término de error en 1 está dada por

$$
\begin{aligned}
u_{i t} & =\mu_{i}+e_{i t} \\
E\left(e_{i t}\right) & =0 \\
E\left(e_{i t}\right) & =0 \text { para } t \neq s
\end{aligned}
$$

La ecuación 1 incluye los siguientes conjuntos de variables :

1. $\Delta d e p_{i t}$ es la variable dependiente, definida como el cambio semanal/diario en los depósitos de los bancos individuales calculados como $\operatorname{logdep}_{i t}-\log _{\operatorname{lop}} \mathrm{p}_{i t-1}$

2. los $W_{t}^{m}$ son los "fundamentos macroeconómicos" que representan cambios en el riesgo agregado. Las variables incluidas en el conjunto (véase gráfico 4) son las siguientes:

- El cambio en el índice de producción industrial ajustado estacionalmente ( $\Delta$ Ind.Prod.), que tiene una frecuencia mensual. Esta variable, solo incluida en el modelo para la muestra total, es un indicador de la posición cíclica de la economía.

- El cambio en las reservas internacionales del Banco Central ( $\Delta$ Rsrvs).

- El spread del EMBI como medida de los cambios en el riesgo país percibido.

- El riesgo de devaluación (Dev.Risk) medido como el spread entre la tasa de interés promedio para los depósitos en pesos y la tasa de interés para los depósitos en dólares.

- El cambio en la liquidez agregada del sistema financiero ( $\Delta$ Sys.Liq.), dado por las tenencias de activos líquidos totales de los bancos, de acuerdo con las regulaciones de liquidez del BCRA. Hubo cambios regulatorios durante este período. $\mathrm{Al}$ comienzo, el BCRA redujo los encajes para dar liquidez a las instituciones financieras. En junio de 2001, los requerimientos de liquidez fueron reemplazados por requerimientos de reservas para los depósitos a la vista. 
3 Las variables $X_{i t}^{k}$ son los fundamentos exógenos de los bancos individuales. Estas variables tratan de capturar hasta qué punto los depositantes eran capaces de discriminar entre bancos, de acuerdo con su solvencia, liquidez y rentabilidad. Las variables en este grupo varían entre individuos y en el tiempo, aunque con una frecuencia menor (mensual) que la variable dependiente, pues las cifras para construir estos cocientes provienen de los balances de los bancos. Los fundamentos microeconómicos incluyen:

- El $R O E$, calculado como el cociente de la utilidad acumulada de 12 meses, generada por intereses y otros ingresos de los bancos, neta de costos operativos y financieros, respecto del patrimonio.

- Un ratio de apalancamiento, construído como el ratio entre los pasivos netos y la riqueza neta.

- Un ratio de activos de riesgo respecto de los activos totales (RiskRatio), donde los activos de riesgo y los activos totales son aquellos considerados por la regulación para el cálculo de los requerimientos de capital.

- El cociente de las tenencias de deuda del gobierno respecto de los activos totales (PubDebt). Esta variable incluye las tenencias de bonos públicos de los bancos, así como los préstamos a los gobiernos nacional, provinciales y municipales. Aunque no es una variable considerada tradicionalmente como fundamental de los bancos, la incluimos por el papel que tuvieron los bancos como principal fuente de financiamiento del gobierno en el caso argentino.

- El cociente de los activos líquidos respecto de los pasivos líquidos (Liquidity), como medida de la liquidez de los bancos individuales.

4 Las $Z_{t}^{r}$ son variables de control. Se usan variables dummy para dar cuenta de la estacionalidad y los efectos "grupo de banco". También se incluyen dummies multiplicativas que dan cuenta de cambios de pendiente, así como una dummy multiplicativa que da cuenta de las asimetrías en el comportamiento de la variable dependiente. Esta última variable toma el valor del cambio en los depósitos en t-1 si el cambio en t es positivo, y cero si no lo es. Un signo negativo para esta variable indica que el proceso autorregresivo es más persistente cuando los depósitos están cayendo que cuando están aumentando (los depositantes se preocupan por la tendencia pasada de los depósitos cuando están cayendo, pero no cuando están aumentando). Los modelos también incluyen dummies diarias estacionales. En el Gráfico \& se presenta la evolución tanto de los depósitos totales en el 
1. Determinantes de las crisis bancarias en las economías emergentes: ¿Fundamentos o profecías autocumplidas? ${ }^{20}$

sistema financiero como los fundamentos macroeconómicos que se incluyen como regresores en la estimación de 1 . 
1. Determinantes de las crisis bancarias en las economías emergentes: ¿Fundamentos o profecías autocumplidas? ${ }^{21}$

Gráficos 4: Fundamentos macroeconómicos
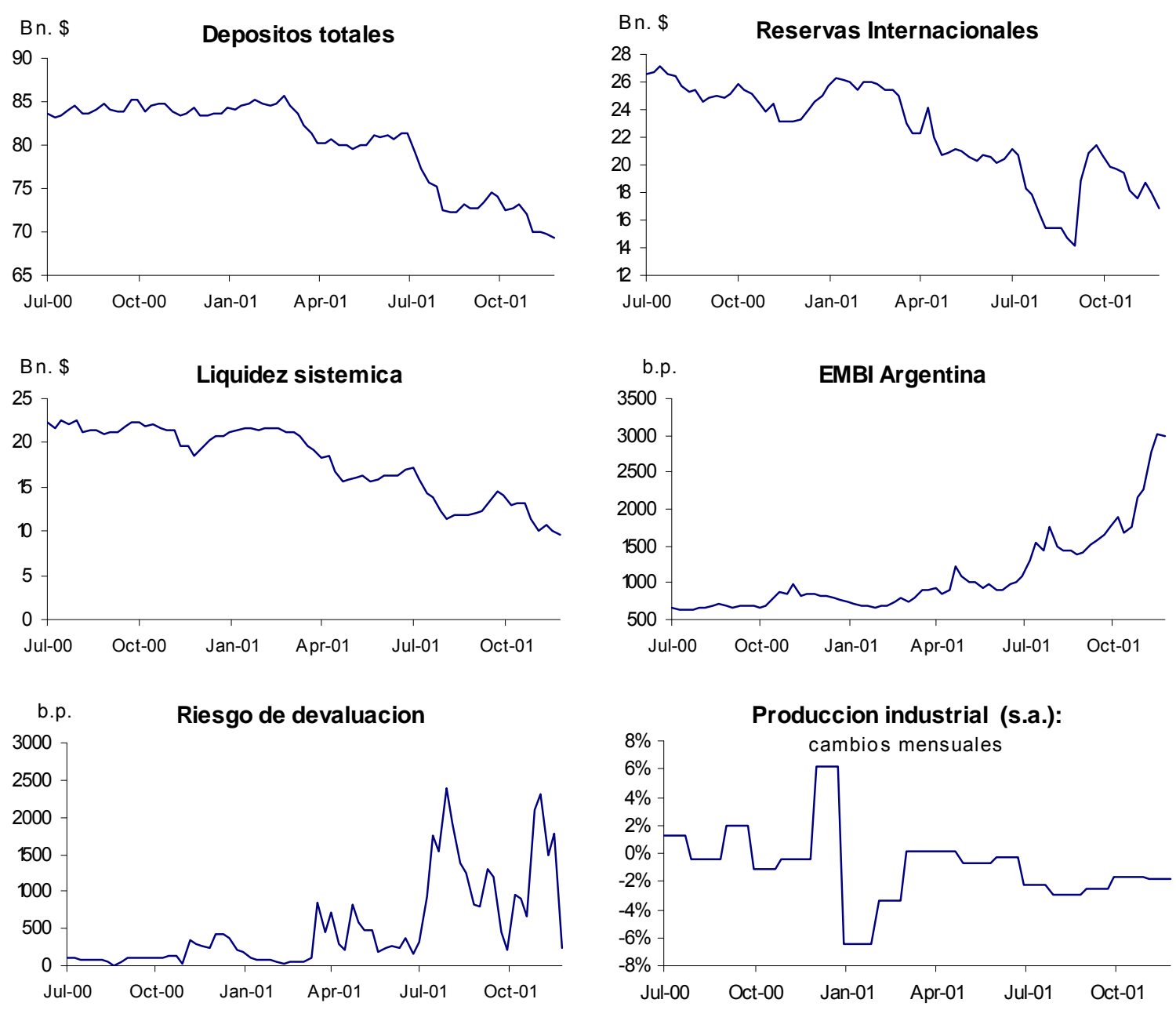

Fuentes: BCRA, INDEC y JP Morgan

\subsubsection{Resultados econométricos}

Como se mencionó anteriormente, estimamos la ecuación 1 , tanto para los cuatro episodios de caída de depósitos indentificados en el análisis descriptivo precedente como para el período completo, julio de 2000-noviembre de 2001.

Nuestra objetivo aqui es evaluar las hipótesis propuestas por la literatura acerca de las causas de las crisis bancarias: (i) son el producto de un mal equilibrio, es decir, hechos aleatorios provocados por percepciones erróenas de los agentes respecto de las necesidades de liquidez de otros agentes; (ii) son un fenómeno de equilibrio, es decir, la respuesta de los agentes económicos al deterioro de los fundamentos macroeconómicos; 
(iii) son un ejercicio de disciplina de mercado por el cual los depositantes castigan el riesgo excesivo asumido por las instituciones financieras.

Para identificar qué factores fueron determinantes en el desarrollo de la crisis bancaria y monetaria de Argentina en 2001, calculamos los modelos de efectos fijos y aleatorios en ambas dimensiones: individuo y tiempo. La ausencia de efectos individuos daría evidencia de que la crisis no fue del tipo (iii), es decir, no fue impulsada por la percepción de los depositantes de un riesgo excesivo asumido por algunos bancos en particular. Por otro lado, si no se presentan efectos tiempo, se puede dejar de lado la hipótesis (ii). Si ninguno de los dos efectos están presentes, este resultado podría considerarse como una evidencia de que (i) es cierta, es decir, las crisis podrían considerarse como el producto de un mal equilibrio.

En primer lugar, trabajamos con modelos autorregresivos para el cambio en la corrida de depósitos, para evaluar la presencia de efectos individuo y efectos tiempo. Se puede ver de la Tabla 3, donde se presentan los resultados de los tests de efectos individuo y tiempo, que sólo en el primer episodio aparecen efectos individuo, que se tornan no significativos una vez que se controla por los fundamentos microeconómicos. Más aún, si se considera toda la muestra, no se encuentran efectos individuo. Así, con excepción de la primera corrida bancaria, parece haber pruebas bastante sólidas de que la crisis no fue impulsada por la debilidad de los bancos individuales. Por el contrario, cuando evaluamos la signficatividad de los efectos temporales, encontramos que son significativos en todos los períodos, excepto el primero. Así, la evidencia parece favorecer la hipótesis de una crisis impulsada por un deterioro de los fundamentos macroeconómicos. Para confirmar esta evidencia, introducimos variables macroeconómicas relevantes que intentan capturar una dinámica común de los depósitos en todos los bancos, dando cuenta así de los efectos tiempo. Se puede observar del la Tabla 3 que cuando introducimos el conjunto de variables macroeconómicas esas variables dan cuenta de los efectos tiempo en el segundo y el tercer períodos. ${ }^{22}$ También se observa que continúa existiendo un efecto tiempo en el cuarto período y para toda la muestra. Es decir, nuestro conjunto de variables macroeconómicas captura parcialmente la dinámica común a los dépositos entre los bancos.

\footnotetext{
${ }^{22}$ En el caso de los efectos de tiempo, una vez introducidos los fundamentos macroeconómicos, que varían en el tiempo, pero no cambian entre los individuos, solo podemos trabajar con modelos de efectos aleatorios. Para testear por los efectos aleatorios, seguimos a Bera, Sosa-Escudero y Yoon (2001), que enfatizan la incapacidad de los tests LM para efectos aleatorios y la correlación serial de distinguir entre ambos problemas de especificación, y proponen otros tests. Realizamos tres tipos de tests: el convencional Breusch-Pagan (1980) para efectos aleatorios, el de Baltagi y Li (1995) para correlación serial de primer orden y el test conjunto de Baltagi y Li (1991) para correlación serial y los efectos aleatorios, y la familia de tests ajustados propuestos por Bera, Sosa-Escudero y Yoon (2001). Por simplicidad, y dado que todos los tests fueron concluyentes en la misma dirección, solo presentamente la versión ajustada de test del test de Breusch-Pagan propuesta por Bera, Sosa-Escudero y Yoon .
} 
Resumiendo, los resultados de este primer paso en la evaluación de las hipótesis propuestas sugieren que, con excepción del primer episodio, la crisis tiene las características de una corrida bancaria impulsada por la percepción de un aumento del riesgo agregado, es decir una corrida basada en los fundamentos macroeconómicos, en lugar de una crisis generada por la percepción de que las instituciones financieras particulares asumían excesivos riesgos. El hecho de que los fundamentos microeconómicos parezcan ser el factor que impulsa el primer episodio de retiro de depósitos sugiere que podría haber un proceso de aprendizaje en el que, en el comienzo, los depositantes usaron información pasada sobre el comportamiento de los bancos individuales en períodos anteriores de stress financiero al tomar decisiones para proteger sus ahorros. 
1. Determinantes de las crisis bancarias en las economías emergentes: ¿Fundamentos o profecías autocumplidas? ${ }^{25}$

Tests de efectos de individuos y tiempo

\begin{tabular}{|c|c|c|c|c|}
\hline & & $\begin{array}{l}\text { Especificación } \\
\text { autorregresiva }\end{array}$ & $\begin{array}{l}\text { Especificación } \\
\text { autorregresiva + } \\
\text { variables macro }\end{array}$ & $\begin{array}{l}\text { Especificación } \\
\text { autorregresiva + } \\
\text { variables micro }\end{array}$ \\
\hline \multirow{4}{*}{ Período completo } & & $F$ test that all $u_{i}=0$ & & \\
\hline & Efectos individuo & $\begin{array}{c}F(26,1895)=0.75 \\
p-\text { value }=0.811\end{array}$ & - & - \\
\hline & & & & \\
\hline & Efectos tiempo & $\begin{array}{c}F \text { test that all } u_{t}=0 \\
F(73,1848)=3.29 \\
p-\text { value }=0.000\end{array}$ & $\begin{array}{c}\text { LM test } s_{\mathrm{u}}=0 \\
\operatorname{Pr}>\operatorname{chi}(1)=0.000\end{array}$ & - \\
\hline \multirow{4}{*}{ Primer episodio } & & $F$ test todos los $u_{i}=0$ & & $F$ test todos los $u_{i}=0$ \\
\hline & Efectos individuo & $\begin{array}{l}F(26,673)=2.85 \\
p \text {-value }=0.000\end{array}$ & - & $\begin{array}{l}F(26,664)=1.07 \\
p \text {-value }=0.3716\end{array}$ \\
\hline & & & & \\
\hline & Efectos tiempo & $\begin{array}{c}F \text { test todos los } u_{t}=0 \\
F(25,674)=0.64 \\
p \text {-value }=0.9135\end{array}$ & $\begin{array}{c}\text { LM test } \mathrm{S}_{\mathrm{u}}=0 \\
\operatorname{Pr}>\operatorname{chi}(1)=0.0914\end{array}$ & - \\
\hline \multirow{4}{*}{ Segundo episodio } & & $F$ test todos los $u_{i}=0$ & & \\
\hline & Efectos individuo & $\begin{array}{l}F(26,889)=0.66 \\
p \text {-value }=0.8983\end{array}$ & - & - \\
\hline & & & & \\
\hline & Efectos tiempo & $\begin{array}{c}F \text { test todos los } u_{t}=0 \\
F(33,882)=1.34 \\
p \text {-value }=0.0991\end{array}$ & $\begin{array}{c}\text { test LM } s_{\mathrm{u}}=0 \\
\operatorname{Pr}>\operatorname{chi}(1)=0.5861\end{array}$ & - \\
\hline \multirow{4}{*}{ Tercer episodio } & & $F$ test todos los $u_{i}=0$ & & \\
\hline & Efectos individuo & $\begin{array}{l}F(26,916)=0.49 \\
p \text {-value }=0.985\end{array}$ & - & - \\
\hline & & & & \\
\hline & Efectos tiempo & $\begin{array}{c}F \text { test todos los } u_{t}=0 \\
F(33,908)=1.69 \\
p \text {-value }=0.009\end{array}$ & $\begin{array}{c}\text { test LM } s_{\mathrm{u}}=0 \\
\operatorname{Pr}>\operatorname{chi}(1)=0.1282\end{array}$ & - \\
\hline \multirow{4}{*}{ Cuarto episodio } & & $F$ test todos los $u_{i}=0$ & & \\
\hline & Efectos individuo & $\begin{array}{c}F(26,1090)=0.26 \\
p-\text { value }=0.999\end{array}$ & - & - \\
\hline & & & & \\
\hline & Efectos tiempo & $\begin{array}{c}F \text { test todos los } u_{t}=0 \\
F(33,908)=2.25 \\
p-\text { value }=0.000\end{array}$ & $\begin{array}{l}\text { test LM } s_{\mathrm{u}}=0 \\
\operatorname{Pr}>\operatorname{chi}(1)=0.001\end{array}$ & - \\
\hline
\end{tabular}

Una vez que hemos determinado que, con excepción del primer período, predomina en la dinámica de los depósitos un efecto común, el segundo paso es calcular un modelo para la ecuación 1 usando el método de Arellano y Bond y un estimador de los efectos fijos, para luego comparar los resultados. En la Tabla 4 presentamos los resultados de ambas estimaciones. Puede verse a primera vista que con excepción del primer período, los fundamentos microeconómicos específicos de los bancos no son significativos. Por el contrario, los fundamentos macroeconómicos adquieren importancia a medida que se va desarrollando la crisis y también son significativos para toda la muestra. 
Tabla 4

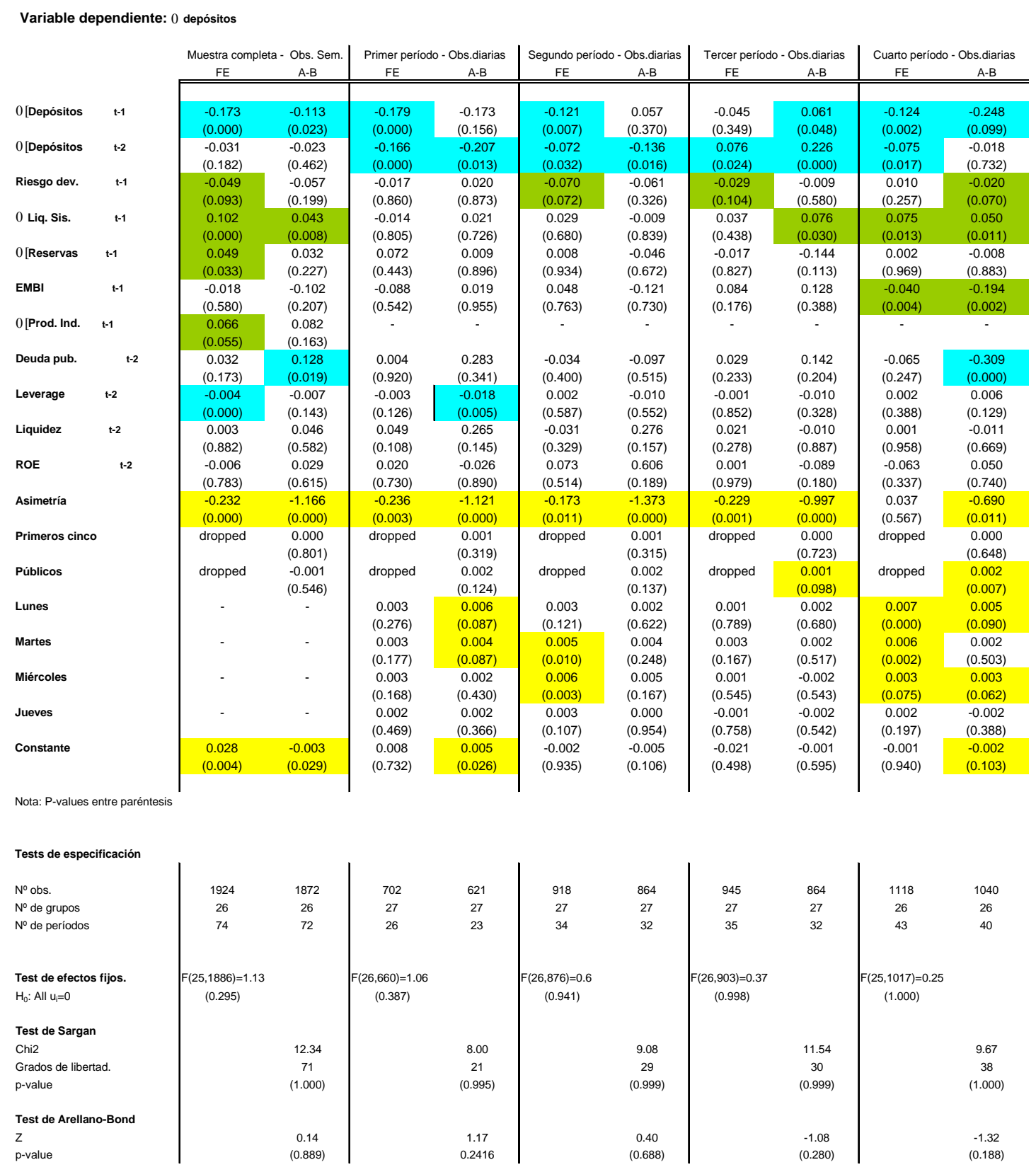

Si nos concentramos en el modelo para todo el período, los resultados son bastante sólidos: los fundamentos microeconómicos de los bancos individuales no son significativos, excepto por el apalancamiento y las tenencias de deuda pública (esta última con el signo inverso al esperado). Por el contrario, todos los fundamentos macroeconómicos, excepto el spread del EMBI (spread del Índice de Mercados Emergentes), son significativos y tiene el signo esperado. Un resultado importante es que el valor rezagado 
del cambio en el índice de producción industrial, una variable que podría considerarse como indicador de una caída futura en el precio de los activos, en el espíritu de Allen y Gale (op. cit.), también es significativa con el signo positivo esperado.

Comparando los modelos a través de los períodos, los resultados refuerzan los obtenidos para toda la muestra: mientras que las variables macroeconómicas son relevantes en todos los períodos, los fundamentos microeconómicos, excepto para el primer período, no parecen tener un papel clave. El riesgo de devaluación es persistentemente significativo en los tres últimos períodos. La liquidez sistémica también es relevante para el tercer y cuarto período, mientras que el spread del EMBI es particularmente significativo en el último período. Consistentemente con los resultados obtenidos en el primer paso, las variables microeconómicas parecen ser relevantes solo en el primer período, en el cual el cociente de apalancamiento y la liquidez de los bancos individuales son significativos y tienen el signo correcto. Los fundamentos microeconómicos pierden importancia en los siguientes episodios. La tenencia de deuda pública, introducida como una medida de exposición de los bancos a la deuda pública, es la única variable específica que resulta significativa en el cuarto período, pues los bancos con mayor exposición a la deuda pública estuvieron sometidos a una corrida más intensa.

Con respecto a los modelos GMM, Arellano y Bond (1991) sugieren dos tests para evaluarlos. El primero, desarrollado por Sargan (1958) y posteriormente por Hansen (1982), evalúa la restricciones de sobreidentificación. Según la hipótesis nula, el conjunto de instrumentos se especifica correctamente. El segundo evalúa la presencia de autocorrelación de segundo orden en la primera diferencia de los residuos, que es esencial para que el estimador GMM sea consistente. Como puede verse en el cuadro 4, todos los modelos superan ambos tests.

En síntesis, la evidencia empírica apoya la hipótesis de que la crisis fue desencadenada por un deterioro de los fundamentos macroeconómicos, en lugar de un pánico bancario causado por el excesivo riesgo asumido por las instituciones financieras o un fenómeno de "manchas solares". Estos resultados demuestran ser bastante robustos a un cambio en la técnica de estimación.

\subsection{Conclusiones}

Desarrollamos una metodología para estudiar empíricamente los determinantes de las crisis financieras que nos permite evaluar la importancia de las tres principales hipótesis que provee la literatura para explicar el fenómeno de las crisis bancarias. La aplicamos al estudio de la dinámica de los depósitos de los bancos individuales durante la crisis gemela sufrida por la Argentina desde julio de 2000 hasta noviembre de 2001. Nuestro objetivo es determinar hasta qué punto esta crisis tuvo las características de un 
fenómeno de "mancha solar" o fue la consecuencia de un deterioro de los fundamentos macroeconómicos de la economía argentina, es decir, un aumento en el riesgo agregado, percibido por los depositantes como una indicación de futuros problemas de solvencia en los bancos.

Los resultados empíricos favorecen fuertemente la segunda hipótesis. Los fundamentos macroeconómicos y, en particular, el riesgo de devaluación y la caída en el nivel de actividad económica, que puede considerarse como un indicador anticipado de un deterioro en la solvencia en los bancos, juegan un papel predominante en explicar la dinámica de los depósitos durante la crisis. La exposición de los bancos individuales a la deuda pública también es significativa en explicar esa dinámica. Por el contrario, los fundamentos microeconómicos de los bancos no contribuyen significativamente a explicarla. También hay evidencia de que esa dinámica adquirió un carácter sistémico a medida que fue desarrollándose la crisis. Hubo ciertas heterogeneidades entre bancos al comienzo, que dejaron de ser significativas con el deterioro de los fundamentos macroecnómicos.

Nuestros hallazgos sugieren que las características perculiares del régimen monetario de la Convertibilidad, que favorecía la percepción de que los deudores sería protegidos en forma permanente contra el riesgo devaluatorio, estimuló la dolarización de la cartera de los bancos, imponiendo un alto riesgo de solvencia al sistema financiero. Ese riesgo no fue percibido como tal hasta que los fundamentos macroeconómicos de la economía no dieron señales de un franco deterioro. La evidencia empírica también sugiere que el marco regulatorio era en ese sentido débil en relación a la exposición del sistema financiero al riesgo de devaluación y también al riesgo de la deuda pública, particularmente en un marco institucional en el que la convertibilidad ponía fuertes restricciones al financiamiento del gobierno.

Algunas recomendaciones de política parecen bastante claras: primero, un elemento clave para permitir una profundización del sistema bancario es desarrollar instrumentos financieros atractivos en moneda local. Segundo, dado que los sistemas financieros están sujetos al riesgo devaluatorio, las regulaciones deben controlar este riesgo. Un buen diseño de regulación debería estar orientado a que : (i) los depositantes internalicen el riesgo implícito en los depósitos en moneda extranjera, pues el Banco Central no tiene instrumentos de política para actuar como un prestamista de última instancia en esa moneda; (ii) poner restricciones a los préstamos bancarios en moneda extranjera, asegurándose que los deudores en esa moneda perciban ingresos denominados en ella, de modo de que su solvencia no se vea afectada por las fluctuaciones del tipo de cambio; (iii) controlar la exposición al riesgo de deuda soberana de la cartera activa de los bancos.

Las últimas experiencias de crisis en mercados emergentes y, más específicamente, 
la crisis argentina de 2001, dejaron en claro que las políticas de liberalización financiera deben ir acompañadas de regulaciones que controlen ampliamente el riesgo de los bancos, e impidan una excesiva expansión del crédito. Las Economías de los Mercados Emergentes, probablemente debido a la falta de ahorro nacional para sostener el crecimiento, dependen de los flujos de capital. En este sentido, sus sistemas bancarios enfrentan riesgos particulares no compartidos por los de economías maduras. En consecuencia, las normas regulatorias para los sistemas bancarios de las EME, deberían lograr un adecuado control de los riesgos relevantes para estas economías, pudiendo quizás podrían diferir en ciertos aspectos de las de los países desarrollados. 
1. Determinantes de las crisis bancarias en las economías emergentes: ¿Fundamentos o profecías autocumplidas? ${ }^{30}$

\subsection{Apéndice A. Correlaciones de Spearman}

\begin{tabular}{|c|c|c|c|c|c|c|}
\hline & \multicolumn{6}{|c|}{ Periodo 1: Desde el 8/11/00 al 13/12/00 } \\
\hline & \multicolumn{6}{|c|}{ Número de observaciones= 26} \\
\hline & depósitos & EMBI & Riesgo dev. & Non Del Fwd & tasa & liq. sist. \\
\hline depósitos & 1 & & & & & \\
\hline EMBI & -0.251 & 1 & & & & \\
\hline Deval Risk & -0.132 & -0.154 & 1 & & & \\
\hline Non Del Fwd & 0.034 & $0.692^{\star \star \star}$ & -0.081 & 1 & & \\
\hline tasa & 0.049 & $-0.339^{\star}$ & 0.155 & $-0.380^{*}$ & 1 & \\
\hline \multirow[t]{4}{*}{ liq. sist. } & 0.064 & $-0.428^{\star *}$ & 0.116 & -0.304 & $0.796^{* \star *}$ & 1 \\
\hline & \multicolumn{6}{|c|}{ Periodo 2: Desde el 12/02/01 al 30/03/01 } \\
\hline & \multicolumn{6}{|c|}{ Número de observaciones $=34$} \\
\hline & depósitos & EMBI & Deval Risk & Non Del Fwd & tasa & liq. sist. \\
\hline depósitos & 1 & & & & & \\
\hline EMBI & -0.284 & 1 & & & & \\
\hline Deval Risk & -0.286 & $0.840^{\star * \star}$ & 1 & & & \\
\hline Non Del Fwd & $-0.362^{\star \star}$ & $0.945^{\star \star \star}$ & $0.875^{\star \star \star}$ & 1 & & \\
\hline tasa & 0.246 & $-0.498^{\star \star \star}$ & $-0.390^{\star *}$ & $-0.439^{\star \star \star}$ & 1 & \\
\hline \multirow[t]{4}{*}{ liq. sist. } & 0.198 & -0.244 & -0.181 & -0.183 & $0.508^{\star \star \star}$ & 1 \\
\hline & \multicolumn{6}{|c|}{ Periodo 3: Desde el 4/07/01 al 23/08/01 } \\
\hline & \multicolumn{6}{|c|}{ Número de observaciones $=35$} \\
\hline & H depósitos & EMBI & Deval Risk & Non Del Fwd & H tasa & H liq. sist. \\
\hline Hdepósitos & 1 & & & & & \\
\hline EMBI & -0.117 & 1 & & & & \\
\hline Deval Risk & -0.047 & $0.522^{* * *}$ & 1 & & & \\
\hline Non Del Fwd & 0.083 & $0.587^{\star \star \star}$ & 0.255 & 1 & & \\
\hline Htasa & $0.428^{\star \star \star}$ & $-0.279^{\star}$ & -0.016 & -0.047 & 1 & \\
\hline H liq. sist. & $0.583^{\star \star \star}$ & -0.211 & -0.015 & -0.027 & $0.796^{\star \star \star}$ & 1 \\
\hline
\end{tabular}

Periodo Completo con datos semanales: Desde el 5/07/00 al 28/11/01 Número de observaciones $=74$

\begin{tabular}{|c|c|c|c|c|c|c|}
\hline & H depósitos & EMBI & Deval Risk & Non Del Fwd & H tasa & H liq. sist. \\
\hline H depósitos & 1 & & & & & \\
\hline EMBI & $-0.228^{*}$ & 1 & & & & \\
\hline Deval Risk & $-0.355^{\star \star \star}$ & $0.843^{\star \star \star}$ & 1 & & & \\
\hline Non Del Fwd & $-0.217^{\star}$ & $0.978^{\star \star *}$ & $0.856^{\star * *}$ & 1 & & \\
\hline H tasa & $0.275^{\star \star}$ & $-0.283^{\star \star}$ & $-0.198^{\star}$ & $-0.301^{\star \star *}$ & 1 & \\
\hline H liq. sist. & $0.457^{* * *}$ & -0.163 & -0.132 & -0.151 & $0.771^{\star \star *}$ & 1 \\
\hline
\end{tabular}




\section{Bibliografía}

[1] Allen, F y D. Gale (1998), "Optimal Financial Crisis", The Journal of Finance, Vol. LIII, n. ${ }^{\circ} 4$.

[2] Allen, F y D. Gale (2000), "Financial Contagion", Journal of Political Economy, Vol. 108, n. ${ }^{\circ} 1$.

[3] Allen. F. (2001), "Financial Structure and Financial Crisis", International Review of Finance, 2:1/2, 2001, 1-19.

[4] Arellano, M. y S. Bond (1991), "Some Tests of Specification for Panel Data: Monte Carlo Evidence and an Application to Employment Equations", Review of Economic Studies, 58, 277-297.

[5] Baltagi, B. (1995), "Econometric Analysis of Panel Data". John Wiley and Sons Ltd.

[6] Baltagi, B. y Q. Lee (1991), "A Joint Test for Serial Correlation and Random Individual Effects," Statistics and Probability Letters, Vol. 11.

[7] Baltagi, B. y Q. Lee (1995), "Testing AR(1) Against MA(1) Disturbance in an in an Error Component Model". Journal of Econometrics 68, 133-151.

[8] Bera, A. K., W. Sosa-Escudero y M. Yoon (2001), "Test for the Error Component Model in the Presence of Local Misspecification", Journal of Econometrics 101, $1-23$.

[9] Breusch, T.S. y A. R. Pagan (1980), "The Lagrange Multiplier Test and Its Application to Model Specification in Econometrics", Review of Economic Studies 47, 239-253.

[10] Broda, C. y E. Levy-Yeyati (2001), "Dollarization and the Lender of Last Resort", Dollarization, MIT Press. 
[11] Bryant, J. (1980), "A model of reserves, bank runs and deposit insurance", Journal of Banking and Finance 4, 335-344.

[12] Calomiris, C. y J. Mason (1994), "Contagion and bank Failures during the Great Depression: The June 1932 Chicago Bank Panic", National Bureau of Economic Research, Documento de trabajo 4934.

[13] Calvo, G., A. Izquierdo y E. Talvi (2003), "Sudden Stops, the Real Exchange Rate, and Fiscal Sustainability: Argentina's Lessons ", NBER Working Paper No. W9828.

[14] Carrera, J.(2002), "Hard Peg and Monetary Unions: Main Lessons from the Argentine Experience", Mimeo.

[15] Chang, R. y A. Velasco (1998), "The Asian Liquidity Crisis", National Bureau of Economic Research, Documento de trabajo Nro. 6796. .

$[16]$ (1999), "Liquidity Crises in Emerging Markets: Theory and Policy", National Bureau of Economic Research, Macroeconomic Annual, The MIT Press.

$[17]$ (2000), "Financial Fragility and the Exchange Rate Regime", Journal of Economic Theory 92, 1-34. .

[18] (2001), "A Model of Financial Crises in Emerging Markets", Quarterly Journal of Economics, Mayo, 489-517.

[19] Chari, V., y R. Jagannathan (1988), "Banking Panics, Information and Rational Expectations Equilibrium", Journal of Finance 43, 749-760. .

[20] D'Amato L., E. Grubisic y A. Powell (1997), "Contagion, Banks Fundamentals or Macroeconomic Shocks? An empirical Analysis of the Argentine 1995 Banking Problems". BCRA, Documento de trabajo Nro.2.

[21] Davidson, R. y J. MacKinnon (1993), "Estimation and Inference in Econometrics", Oxford University Press.

[22] Della Paolera, G y A. Taylor (2003), "Gaucho Banking Redux", Fundación PENT Documento de Trabajo.

[23] Diamond, D. y P. Dyvbig (1983), "Bank Runs, Deposit Insurance and Liquidity", Journal of Political Economy 91, 401-419. 
[24] Diaz Alejandro, C.F. (1985), "Good bye Financial Repression, Hello Financial Crash", Journal of Development Economics 19.

[25] Ennis, H. (2002), "Economic Fundamentals and Bank Runs", Federal Reserve Bank of Richmond, Economic Quarterly (10697225), Spring2003, Vol. 89 Issue 2.

[26] Forbes, K. y R. Rigobon. (1999), "No Contagion, only Interdependence : measuring Stock Market Co- movement". National Bureau of Economic Research, Documento de trabajo 7267 .

$[27]$ (1999), "Measuring Contagion: Conceptual and Empirical Issues", Mimeo.

[28] Freixas, X. y J.C. Rochet (1998), "Microeconomics of Banking", The MIT Press.

[29] Galeani, S., D. Heymann y M. Tommasi (2003), "Expectativas frustradas: El ciclo de la convertibilidad", CEPAL. Serie Estudios y Perspectivas Nro 16.

[30] Gorton, G.(1985), "Banks Suspension of Convertibility", Journal of Monetary Economics, 15: 177-93.

[31] Hansen, L. (1982), "Large Sample Properties of Generalized Method of Moments Estimators", Econometrica 50, 1029-1024.

[32] Hansen L. y K. Singleton (1982), "Generalized Instrumental Variables Estimation of Nonlinear Rational Expectations Models", Econometrica 50, 267-268.

[33] Hausmann, R. y A. Velasco (2002), "Hard Money's Soft Underbelly: Understanding the Argentine Crisis", Mimeo.

[34] Hellwig, M. (1994), "Liquidity provisioning, banking, and the allocation of interest rate risk". European Economic Review 38, 1363-1389.

[35] Jacklin, Ch .J. y S. Bhattacharya (1988), "Distinguishing Panics and Informationbased bank runs: Welfare and Policy implications", Journal of Political Economy 96, 586-592.

[36] Judson R. y A. Owen (1996), "Estimating Dynamic Panel Data Models: A Practical Guide for Macroeconomists". Federal Reserve Board of Governors.

[37] Kaminsky, G. y C. Reinhart (1999), "The Twin Crises: The Causes of Banking and Balance of Payments Problems", The American Economic Review, junio, 473-500.

[38] Kawamura, E. (2002), "Banks with Peso-Denominated Deposits in Small Open Economies with Aggregate Liquidity Shocks", Mimeo UdeSA. 
[39] Kiviet, J. (1995), "On bias inconsistency, and efficiency of various estimators in dynamic panel data models", Journal of Econometrics 68, 53-78.

[40] Park, S. (1991), "Bank Failure Contagion in Historical Perspective", Journal of Monetary Economics, 28, 271-286..

[41] Perry, G. y L. Serven (2003), "The Anatomy of a Multiple Crisis: Why was Argentina special and what can we learn from it", World Bank Policy Research Working Paper No. 3081

[42] Powell, A. (2002), "Argentina's Avoidable Crisis: Bad Luck, Bad Economics, Bad Politics, Bad Advice", CIF-UTDT, Documento de Trabajo Nro. 7 año 2002.

[43] Sargan, J.D. (1958), "The Estimation of Economic Relationships Using Instrumental Variables", Econometrica 26, 393-415.

[44] Valdés, R. (1997), "Emerging markets Contagion: Evidence and Theory", Banco Central de Chile, Documento de trabajo Nro $\%$.

[45] Wallace, N. (1988), "Another attmpt to explain an illiquid banking system: The Diamond and Dybig model with sequential service taken seriously", Federal Bank of Minneapolis Quarterly Review 12 (4):3-16.

[46] Wallace, N. (1990), "A banking model in which suspension is best", Federal Reserve Bank of Minneapolis Quarterly Review 14 (Fall), 11-23. 


\section{Capítulo 2}

\section{Dinámica inflacionaria, persistencia y cambio de régimen}

\subsection{Motivación}

Una adecuada comprensión de la dinámica inflacionaria es indispensable para la modelación y la formulación de la política monetaria. La evidencia en la literatura reciente sugiere que con el descenso de la inflación en el mundo, fenómeno atribuible en una medida importante a mejoras sustanciales en las políticas fiscal y monetaria, la dinámica de la inflación ha cambiado. En particular su persistencia, definida ésta como la velocidad con que la inflación se aproxima a su valor de largo plazo luego de un shock, parece haberse reducido. Este fenómeno ha sido ampliamente estudiado en los años recientes para las economías industriales, pero la evidencia para países en desarrollo es bastante más limitada.

Durante muchos años la alta persistencia de la inflación ha sido un hecho estilizado prevaleciente en la literatura, considerándosela como un proceso cercano al de un camino aleatorio. ${ }^{1}$ Este rasgo de la inflación tiene implicancias importantes para la modelación y el accionar de la política monetaria. Los modelos neo-keyesianos en los que suele basarse la modelación de la política se han asentado inicialmente en la presencia de rigidez en el ajuste de los precios para generar un efecto de los shocks nominales sobre la economía real. Debido a que la evidencia empírica sugería que la inflación es altamente persistente y que ese componente inercial genera altos costos de desinflar las economías en términos de ajuste del producto, se ha tendido a introducir persistencia en la inflación. Sin embargo, las desinflaciones pueden ser procesos costosos por falta de credibilidad de los bancos centrales y también por el hecho de que las

\footnotetext{
${ }^{1}$ Ver por ejemplo Furher y Moore (1995), Galí y Gertler (1999), Furher (2006) y también Walsh (2003), para una muy buena revisión de la literatura de referencia.
} 
expectativas pueden no ser perfectamente racionales. ${ }^{2}$

La evidencia internacional reciente sugiere que una vez que se toma en cuenta que el valor de largo plazo de la inflación no es constante, el grado de persistencia se reduce. También hay cierta evidencia de que la persistencia ha sido más elevada en períodos de alta inflación. ${ }^{3}$

El supuesto de un valor de largo plazo constante es sin duda implausible para Argentina, un país que experimentó inflación elevada y persistente en décadas de los 70 `s y 80 's, en las que la política monetaria estuvo fuertemente condicionada por desequilibrios fiscales persistentes que implicaban una elevada dominancia fiscal. Ese período de alta inflación derivó hacia fines de la década de los 80's en un episodio hiperinflacionario, luego del cual se adoptó un régimen monetario de caja de conversión, la Convertibilidad, en el que la política monetaria era escencialmente pasiva y la dinámica inflacionaria estaba gobernada, en gran medida, por factores externos. La inflación se mantuvo en niveles reducidos durante ese período, que finalizó con el abandono de ese régimen en enero de 2002. Luego de la abrupta devaluación del peso, la inflación se aceleró, alcanzando un pico en abril de 2002, para luego retornar a valores más reducidos, aunque superiores a los niveles promedio durante la Convertibilidad.

En este capítulo estudiamos la dinámica inflacionaria y en particular el fenómeno de la persistencia inflacionaria en Argentina en el período 1980-2007. Comenzamos por realizar un ejercicio de simulación para estudiar las implicancias de incorporar ad-hoc un comportamiento backward looking en un modelo neo-keynesiano sencillo. En particular, evaluamos las consecuencias de distintos niveles de persistencia inflacionaria en términos del impacto contractivo de una desinflación. Luego nos concentramos en el estudio de la persistencia inflacionaria en Argentina. Analizamos la vinculación entre los cambios observados tanto en la tasa media de inflación como en su dinámica autorregresiva y los cambios de régimen monetario. Identificamos la presencia de cambios de régimen a través de un análisis recursivo y de la implementación de tests de quiebres múltiples propuestos por Bai y Perron (2003). También contruímos medidas de persistencia que incorporan cambios en la tasa media de inflación y en su componente autorregresivo. El capítulo esta estructurado del siguiente modo: en la sección 2 estudiamos las implicancias de la persistencia inflacionaria en el marco de un modelo neo-keynesiano sencillo. En la sección 3, presentamos los rasgos más relevantes de la dinámica inflacionaria en Argentina en el período bajo estudio: 1980-2007. En la sección 4 presentamos los resultados del análisis descriptivo. En la sección 5 evaluamos la presencia de quiebres en la tasa media de inflación y su vinculación con cambios en el régimen monetario. En la sección 6 calculamos distintas medidas de persistencia

\footnotetext{
${ }^{2}$ Ver al respecto Roberts (1996).

${ }^{3}$ Ver al respecto Altíssimo et al. (2006), Marques, (2004) y Levin y Pigier (2004) entre otros.
} 
inflacionaria. Finalmente, en la sección 7 presentamos la conclusiones.

\subsection{El fenómeno de la persistencia inflacionaria en la lit- eratura: Formación de precios y persistencia de la inflación}

El hecho de que la tasa media de inflación pueda evolucionar experimentando quiebres discretos puede sustentarse en los determinantes de largo plazo de la tasa de inflación. Existe bastante acuerdo en la teoría monetaria acerca de que en el largo plazo la inflación debería estar cointegrada con la tasa de expansión del dinero, de modo que en el estado estacionario la tasa de crecimiento del dinero iguale a la tasa de inflación. ${ }^{4}$ En ese sentido, el valor de estado estacionario de la inflación y el crecimento monetario no son independientes del accionar del banco central en su objetivo de estabilizar a la inflación en torno a ese valor, ni de los condicionamientos que enfrenta para lograrlo. El valor de largo plazo de la inflación puede no ser único, sino en todo caso dependiente del régimen monetario ${ }^{5}$. Al respecto, la evidencia empírica indica que bajo regímenes no ricardianos, con alta dominancia fiscal, la tasa media de inflación es elevada, como también lo es la tasa de expansión monetaria. ${ }^{6}$ Por el contrario, una vez que la política monetaria está menos condicionada por la política fiscal y tiene mayor capacidad de cumplir su rol de proveer una ancla nominal a la economía, la tasa media de inflación tiende a reducirse. También es posible que las economías experimenten tasas de inflación persistentemente elevadas si existe un problema de inconsistencia temporal, que genera incentivos a la política monetaria para sorprender al público con mayor inflación, con el objetivo de explotar el trade-off entre crecimiento e inflación.

Sin embargo, la relación entre la tasa media de inflación y la volatilidad macroeconómica no ha sido incorporada a la modelación macroeconómica en los modelos neo-keynesianos, que en general suponen una tasa de inflación cero para el estado estacionario y no discuten los efectos de inflaciones de largo plazo o de tendencia (trend inflation) positivas y eventualmente elevadas. Recientemente Kiley (2007), Ascari y Ropele, (2007) y Blake y Fernández-Corugedo(2006), entre otros, discuten las implicancias en términos de indeterminación de los equilibrios y volatilidad macroeconómica de inflaciones de largo plazo moderadas o altas. Esta literatura reciente muestra que el permitir una inflación de estado estacionario distinta de cero cambia

\footnotetext{
${ }^{4}$ Ver la respecto Walsh (2003), op.cit.

${ }^{5}$ Heymann y Leijonhufvud (1995) definen en general un régimen de política como el patrón de comportamiento de las autoridades económicas sustenta el sistema de expectativas que gobierna las decisiones del sector privado.

${ }^{6}$ Ver Heymann y Leijonhufvud (1995) y Walsh (2003), op. cit, cap. 4 para una discusión detallada de las relaciones entre la política monetaria y la fiscal.
} 
sustancialmente la dinámica de la inflación, que pasa a ser endógena a la trend inflation. Aparece también como una variable relevante en la determinación de la dinámica inflacionaria la dispersión de precios relativos. Ascari y Ropele (2007), encuentran que cuando se toma en cuenta la posibilidad de una trend inflation distinta de cero, el proceso de formación de precios se torna más forward looking, por lo que la relación contemporánea entre la inflación y la brecha del producto se debilita. Finalmente, una trend inflation más elevada lleva a que el componente autorregresivo en la ecuación que describe la dinámica de la dispersión de precios relativos se incremente, llevado a un sendero de ajuste de la inflación más persistente. Estos resultados parecen estar en línea con la idea de que en contextos de inflación de largo plazo más elevada, la dinámica inflacionaria adquiere una mayor persistencia.

Por otro lado, en los modelos de formación de precios que dan fundamento microeconómico a la modelación macroeconómica actual, como el de Calvo (1983) y el de Taylor (1980), la persistencia de la inflación se explica por la persistencia del principal factor que gobierna su dinámica, que es la brecha del producto, la que suele considerarse una proxy de los costos marginales reales. Sin embargo, distintas especificación de la curva de Phillips en su versión neo-keynesiana, enfrentan la dificultad de que empíricamente el término en la brecha del producto es escasamente significativo ${ }^{7}$. Por otro lado la inflación no es, en esos modelos, intrínsecamente persistente, aunque sí lo es el nivel de precios. Este rasgo tampoco se condice con la evidencia empírica, que indica que las desinflaciones suelen ser episodios bastante costosos en términos de caída del producto. ${ }^{8}$

Al mismo tiempo, la evidencia empírica sugería, al menos hasta hace algún tiempo, que la inflación es un proceso altamente persistente. Por esa razón autores como Furher y Moore (1995) y Galí y Gertler (1999) proponen incorporar un componente intrínseco de persistencia a la inflación.

En particular, Furher y Moore (1995a y 1995b) modifican el modelo de Taylor (1980) en el que los contratos salariales se renegocian en forma escalonada para introducir persistencia intrínseca a la inflación. Una versión simplificada del modelo de Furher y Moore (op. cit.), desarrollada por Walsh (2003), sirve para ilustrarlo. El principal cambio que introducen Furher y Moore al modelo de Taylor es suponer que las decisiones que guían la negociación de salarios se basan en los salarios reales. Debido a que en este modelo los precios son un mark-up sobre los salarios, el nivel de precios queda determinado por la negociación salarial.

En una economía en la que los precios se fijan de acuerdo a un mark-up sobre los

\footnotetext{
${ }^{7}$ Ver al respecto Furher y Moore (1995a, 1995b), Rudd y Whelan (2006) y Furher (2006). En el caso de Argentina, D'Amato y Garegnani estiman un curva de Phillips híbrida y encuentran un coeficiente de 0.016 para la brecha del producto utilizando datos de frecuencia mensual.

${ }^{8}$ Ver al respecto Erceg y Levin (2002)
} 
costos, los contratos salariales pueden considerarse el principal determinante del nivel del nivel general de precios Para negociar salarios, los agentes consideran el valor real del contrato salarial, dado por

$$
c_{t}=x_{t}-p t
$$

donde $c_{t}$ es una medida del valor real del contrato salarial, $x_{t}$ es el salario nominal fijado según el contrato salarial establecido en $t$ y $p_{t}$ es el nivel general de precios.

Suponiendo que los contratos salariales se negocian cada dos períodos, el salario real medio en $t$ estará dado por

$$
\bar{c}_{t}=\frac{1}{2}\left(c_{t}+c_{t-1}\right)
$$

El objetivo de los agentes es obtener un contrato salarial que iguale el valor esperado del contrato promedio corregido por alguna medida de la posición cíclica de la economía dada por el término en el producto $\kappa y_{t}$ en (3)

$$
c_{t}=\frac{1}{2}\left(\overline{c_{t}}+E_{t} \bar{c}_{t+1}\right)+\kappa y_{t}
$$

aplicando el operador esperanza a 2 y adelantando un período se obtiene

$$
E_{t} \bar{c}_{t+1}=\frac{1}{2}\left(c_{t}+E_{t} c_{t+1}\right)
$$

y se puede reemplazar en la ecuación 3 para obtener

$$
c_{t}=\frac{1}{2}\left(\frac{1}{2}\left(c_{t}+c_{t-1}\right)+\frac{1}{2}\left(c_{t}+E_{t} c_{t+1}\right)\right)+\kappa y_{t}
$$

que puede escribirse como

$$
c_{t}=\frac{1}{2}\left(c_{t-1}+E_{t} c_{t+1}\right)+2 \kappa y_{t}
$$

y finalmente, tomando en cuenta 1, la ecuación anterior se puede escribir como

$$
x_{t}-p_{t}=\frac{1}{2}\left[x_{t-1}-p_{t-1}+E_{t}\left(x_{t+1}-p_{t+1}\right)\right]+2 \kappa y_{t}
$$

La ecuación anterior se puede reescribir en variaciones para obtener una expresión en términos de la tasa de inflación

$$
\Delta x_{t}=\frac{1}{2}\left(\pi_{t}+E_{t} \pi_{t+1}\right)+2 \kappa y_{t}
$$

Teniendo en cuenta que en este contexto los precios son un mark-up sobre los costos, el nivel de precios es asimilable al contrato nominal promedio de la economía

$$
p_{t}=\frac{1}{2}\left(x_{t}+x_{t-1}\right)
$$


se puede ver que la tasa de inflación es

$$
\pi_{t}=\frac{1}{2}\left(\Delta x_{t}+\Delta x_{t-1}\right)
$$

por lo que la ecuación (16) puede usarse para escribir la siguiente ecuación para la tasa de inflación

$$
\pi_{t}=\frac{1}{2}\left(\pi_{t-1}+E_{t} \pi_{t+1}\right)+\kappa\left(y_{t}+y_{t-1}\right)+\eta_{t}
$$

Donde el término en $\eta_{t}$ es el error de pronóstico sobre $\pi_{t}$, dado por $-\left(\pi_{t}-E_{t-1} \pi_{t}\right)$.

De este modo se obtiene una curva de Phillips que otorga lentitud al ajuste de la inflación en respuesta a nueva información. En esta especificación, la naturaleza adaptativa de la dinámica inflacionaria implica que las contracciones monetarias serán costosas en términos de caídas del nivel de actividad.

Esta curva de Phillips, que incorpora la inflación pasada para acercar el modelo a los datos observados puede considerarse en el contexto de un modelo de política monetaria convencional que incluya una ecuación de demanda y una regla de política.

En forma general, y para una economía cerrada se tendría un sistema como el que sigue

$$
\begin{gathered}
\pi_{t}=\theta E_{t} \pi_{t+1}+(1-\theta) \pi_{t-1}+\kappa y_{t}+\eta_{t} \\
y_{t}=\rho y_{t-1}-\beta\left(i_{t}-E_{t} \pi_{t+1}\right)+\varepsilon_{t} \\
i_{t}=\gamma i_{t-1}+(1-\gamma)\left(\alpha_{\pi} \pi_{t}+\alpha_{y} y_{t}\right)+\mu_{t}
\end{gathered}
$$

La curva de Phillips en la ecuación 8 puede incluir un componente en la inflación rezagada, como sugiere el anterior modelo de formación de precios o la curva de Phillips híbrida propuesta por Galí y Gertler (1999), que incorpora una regla de formación de precios adaptativa al modelo de Calvo (1983). Furher (2006) muestra alguna evidencia que sugiere que la incorporación de un componente inercial no modifica sustancialmente la persistencia que esto modelos generan sobre la tasa de inflación. El término en $\eta_{t}$ representa un shock sobre la tasa de inflación.

La curva $I S$ (ecuación 9) suele especificarse en las expectativas sobre el producto, pero se puede ver que en ese caso se requiere especificar los shocks sobre la demanda agregada como AR(1) para obtener alguna persistencia en los efectos de los shocks que se asemeje en alguna medida a la observada empíricamente. De ese modo en 9 se tiene que 


$$
\varepsilon_{t}=\rho \varepsilon_{t-1}+\nu_{t} \varepsilon_{t}, \quad \varepsilon_{t} \sim r b
$$

Finalmente, la regla de política puede especificarse siguiendo la regla de Taylor, con $\alpha_{\pi}=1,5$, reflejando que el banco central busca afectar a la tasa real frente a la presencia de presiones inflacionarias y $\alpha_{y}=0,5$, mientras que el término en $\mu_{t}$ puede interpretarse como una innovación sobre la regla de política o eventualmente algún desvío respecto de la regla óptima.

Se puede ver que en su forma reducida, la ecuación 8, que describe la dinámica de la inflación, depende del componente inercial del producto, de la regla de política monetaria, cuyos efectos sobre ella pueden variar según la importancia que el banco central asigne a estabilizar la inflación relativa al producto y de los shocks sobre la propia tasa de inflación, que identificamos como shocks de oferta en este modelo. También depende, como enfatizan Furher y Moore (1995) y Furher (2006), de la dinámica autorregresiva del producto.

$$
\pi_{t}=\left(\theta \lambda+\frac{\beta}{\delta}\right) E_{t} \pi_{t+1}+(1-\theta) \lambda \pi_{t-1}-\frac{\kappa}{\delta} \rho y_{t-1}-\frac{\kappa \beta \gamma}{\delta} i_{t-1}-\frac{\kappa \beta}{\delta} \varepsilon_{t}+\frac{\varepsilon_{t}}{\delta} \lambda \eta_{t}
$$

con

$$
\begin{gathered}
\lambda=\frac{1-\beta(1-\gamma) \alpha_{y}}{\left[1-\beta(1-\gamma)\left(\alpha_{\pi}+\alpha_{y}\right)\right]} \\
\delta=1-\beta(1-\gamma) \alpha_{y}
\end{gathered}
$$

Un ejercicio sencillo de simulación sirve para tener en cuenta los efectos de cambios en la especificación de las ecuaciones 8, 9 y 10 sobre la persistencia inflacionaria. Calculamos los efectos de un shock de demanda bajo distintas especificaciones de la curva de Phillips y distintos régimenes monetarios definidos en base al peso relativo de la inflación a la brecha del producto en la regla de Taylor. En ese sentido definimos en forma implícita a los regímenes monetarios de acuerdo a la regla de política adoptada por el del banco central, siguiendo a Ascari y Ropele (2007).

Partimos de una especificación forward looking de 8 con un coeficiente $\kappa$ de 0,08 para la brecha del producto y suponiendo que los shocks sobre el producto son altamente persistentes $(\rho=0,85)$. Analizamos el impacto de un impulso monetario sobre la tasa de interés, la inflación y el producto. Observamosen el Gráfico1 que la desinflación no genera costos en términos de caída del producto en esta especificación. 
Gráfico 1: respuestas de la tasa de interés, el producto y los precios a un impulso monetario según modelo 1

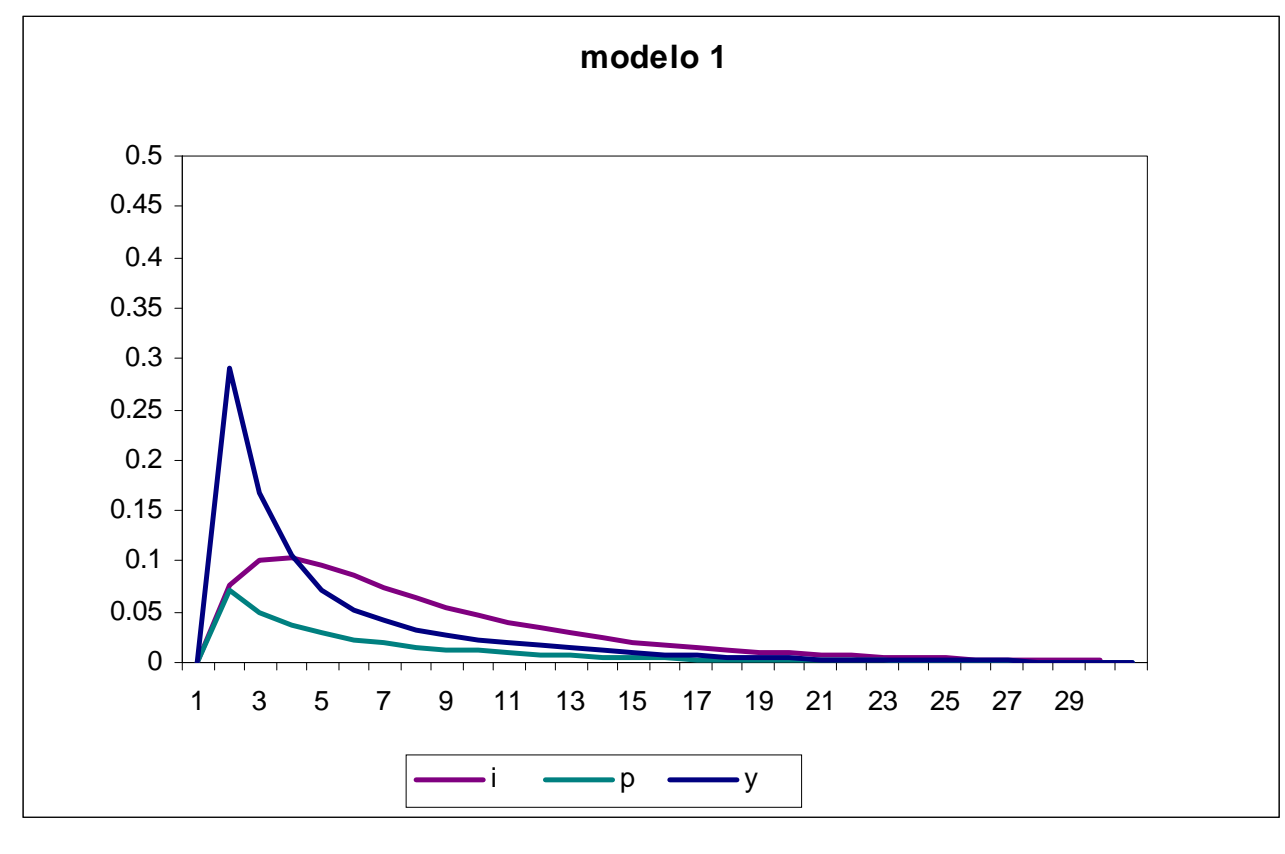

$$
\kappa=0,08, \rho=0,85
$$

Comparamos estos resultados con los efectos de un shock de demanda en una especificación híbrida como en 8 con igual coeficiente para la brecha del producto y con un mayor peso para el componente forward looking de la inflación, $(\theta=0,6)$. Observamos en el Gráfico 2 que con estos pesos relativos de los componentes forward y backward looking de la inflación no se observa un impacto negativo de la desinflación sobre el producto. 
Gráfico 2: respuestas de la tasa de interés, el producto y los precios a un impulso monetario según modelo 2

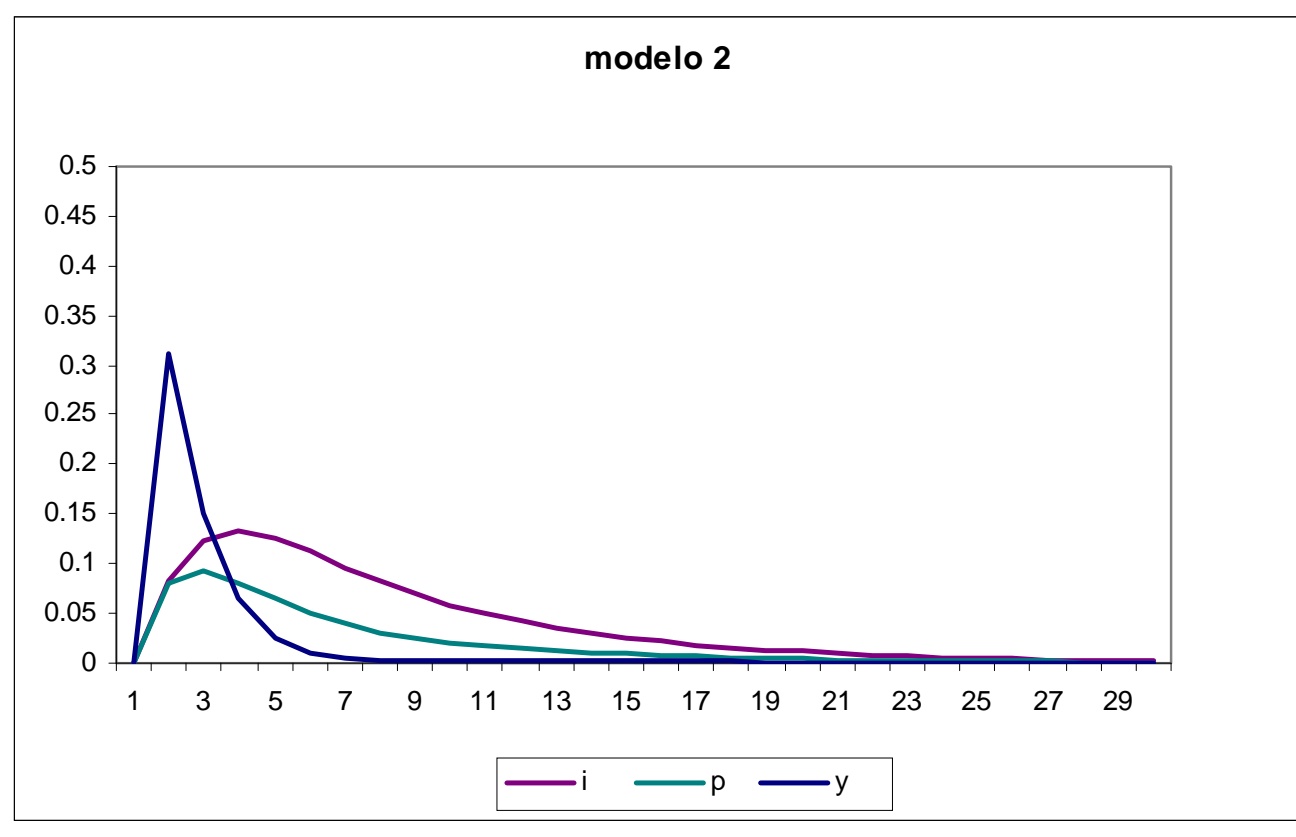

$$
\kappa=0,08, \rho=0,85, \theta=0,6
$$

$\mathrm{Al}$ asignar un mayor peso al componente backward looking de la inflación $((1-\theta)=$ 0,5, la desinflación que sigue al impulso monetario positivo sobre la demanda agregada tiene un efecto negativo sobre la brecha del producto (Gráfico 3 ).

Gráfico 3: respuestas de la tasa de interés, el producto y los precios a un impulso 
monetario según modelo 3

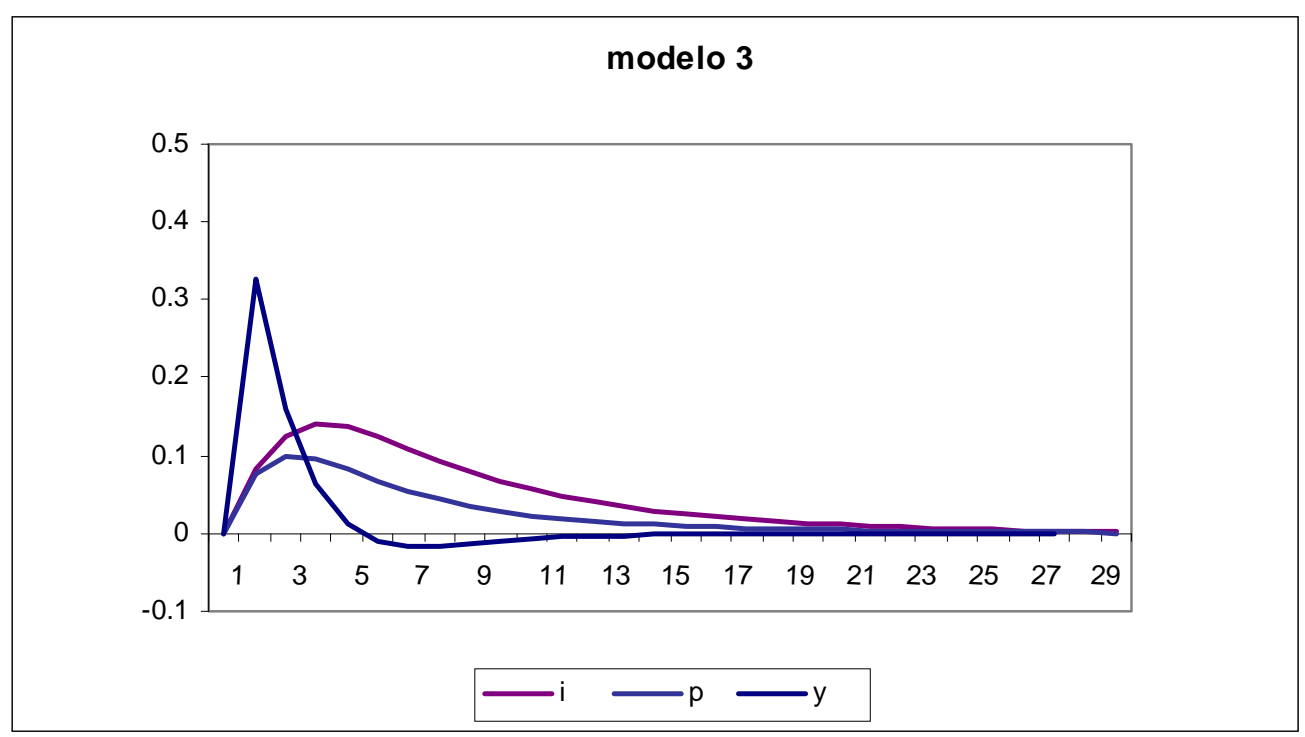

$\kappa=0,08, \rho=0,85,1-\theta=0,5$

Finalmente, si cambiamos la regla de Taylor suponiendo un coeficiente de 0,7 para inflación en la regla de Taylor, sugiriendo que el banco central está dispuesto a tolerar una inflación más elevada, en la medida que no responde a los shocks de demanda aumentando la tasa real, se observa en Gráfico \& un impacto más significativo del impulso positivo sobre la inflación, que además se vuelve más persistente. En ese sentido, corroboramos que un régimen monetario que tolera tasas de inflación más elevadas, genera dinámicas inflacionarias más persistentes. 
Gráfico 4: respuestas de la tasa de interés, el producto y los precios a un impulso monetario según modelo 4

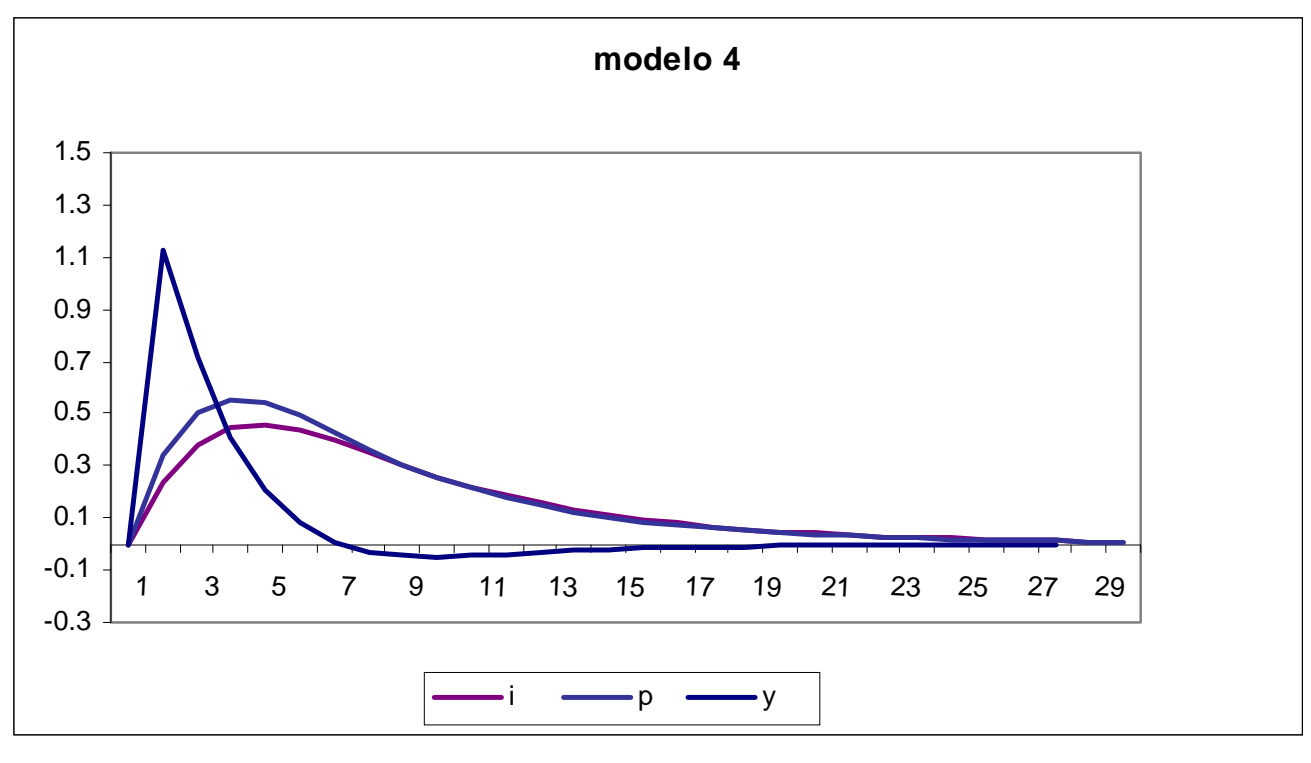

$\kappa=0,08, \rho=0,85,1-\theta=0,6, \alpha_{\pi}=0,7$

La Tabla 1 muestra las autocorrelaciones para las trayectorias de la inflación en respuesta a un shock de demanda para las tres especificaciones propuestas. Se observa que la inflación se torna más pesistente en la especificación híbrida y que también un régimen monetario con mayor tolerancia a la inflación genera una dinámica que se acerca a la de un camino aleatorio. 
Tabla 1

\begin{tabular}{rrrrr}
\multicolumn{5}{c}{ autocorrelaciones } \\
$\mathrm{t}$ & modelo 1 & modelo 2 & modelo 3 & modelo 4 \\
1 & 0.709 & 0.87 & 0.908 & 0.945 \\
2 & 0.55 & 0.701 & 0.767 & 0.847 \\
3 & 0.442 & 0.552 & 0.625 & 0.735 \\
4 & 0.362 & 0.438 & 0.503 & 0.625 \\
5 & 0.301 & 0.352 & 0.404 & 0.524 \\
6 & 0.251 & 0.288 & 0.327 & 0.436 \\
7 & 0.21 & 0.239 & 0.267 & 0.361 \\
8 & 0.176 & 0.199 & 0.219 & 0.298 \\
9 & 0.147 & 0.166 & 0.181 & 0.246 \\
10 & 0.122 & 0.138 & 0.15 & 0.202 \\
11 & 0.101 & 0.115 & 0.124 & 0.166 \\
12 & 0.083 & 0.094 & 0.102 & 0.135 \\
13 & 0.068 & 0.077 & 0.083 & 0.11 \\
14 & 0.055 & 0.062 & 0.067 & 0.088 \\
15 & 0.043 & 0.049 & 0.052 & 0.069 \\
16 & 0.033 & 0.037 & 0.04 & 0.053 \\
17 & 0.025 & 0.027 & 0.03 & 0.04 \\
18 & 0.017 & 0.019 & 0.02 & 0.028 \\
19 & 0.011 & 0.012 & 0.012 & 0.017 \\
20 & 0.005 & 0.005 & 0.005 & 0.008
\end{tabular}

Roberts (1997) y también Erceg y Levin (2002), sugieren que una razón que explicaría la persistencia de la inflación en contextos de inflación elevada en los que el banco central lleva adelante una desinflación es que en un contexto de ese tipo las expectativas pueden no ser perfectamente racionales, sino tener un comportamiento de aprendizaje adaptativo.

\subsection{Algunos rasgos de la dinámica inflacionaria en Ar- gentina}

Como remarcamos inicialmente, el supuesto de un valor de equilibrio constante para la tasa de inflación no es razonable en el caso de Argentina. Al respecto, Capistrán y Ramos-Francia (2006), proveen evidencia sobre persistencia de la inflación para los diez países más grandes de América Latina y encuentran que en el caso argentino el grado de persistencia de la inflación se habría reducido entre 1980 y 2006.

De hecho, como lo ilustra el Gráfico 5, la simple inspección visual sugiere una tasa media de inflación que cambia sustancialmente en el período analizado. 
Gráfico 5: tasa de inflación mensual

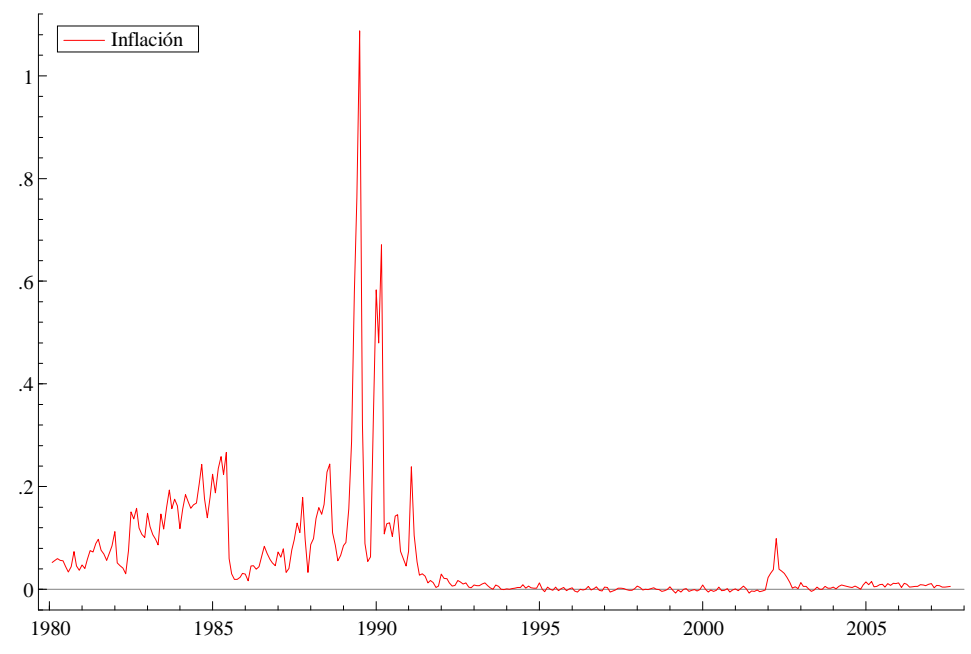

Fuente:INDEC

La inflación elevada fue un fenómeno ampliamente extendido en la región durante los 70's y 80's. El financiamiento monetario de los desequilibrios fiscales fue un rasgo común entre los países que pasaron por episodios hiperinflacionarios como Argentina. Este fenómeno ha recibido un renovado interés en los años recientes. En particular, Sargent, et al (2006) han estudiado la dinámica inflacionaria en los países de la región que experimentaron hiperinflaciones en un modelo de aprendizaje que permite un cambio de expectativas racionales a expectativas adaptativas. Ellos encuentran que en alta inflación la dinámica inflacionaria en esos países reproduce en gran medida la sugerida por Cagan (1956). ${ }^{9}$ Concluyen que estas economías lograron estabilizar la inflación de manera permanente en la medida que lograron una disciplina fiscal.

El caso argentino tiene, sin embargo, características peculiares. La inflación comenzó a ser persistentemente elevada desde mediados de los años 70’s, período en que el sector público mantuvo elevados déficits presupuestarios. Hacia fines de esa década se adoptó un crawling peg con el dólar, esquema que intentaba una convergencia de la inflación doméstica a la internacional. En esos años, al igual que otras economías de la región, Argentina inició un proceso de liberalización comercial y financiera. El peso sufrió una creciente apreciación real, al tiempo que la economía experimentaba persistentes déficit de cuenta corriente. El alza de la tasa de interés internacional en

\footnotetext{
${ }^{9}$ Basco et al.(2006) encuentran similares resultados al estudiar la dinámica de la relación dineroprecios en alta inflación.
} 
1982 llevó a una severa crisis de deuda en la región, que afectó fuertemente a Argentina. El peso fue devaluado y el gobierno se hizo cargo de una parte importante de la deuda externa privada, lo que amplió los desequilibrios fiscales preexistentes. En los años que siguieron la inflación fue acelerándose marcadamente. En 1985 hubo un intento de estabilización conocido como el Plan Austral, que sólo logró una estabilización temporaria de la inflación, luego de lo cuál la inflación se aceleró fuertemente hasta desembocar en un episodio hiperinflacionario a mediados de 1989.

En abril de 1991, la implementación de un esquema de caja de conversión y la fijación por ley del peso al dólar estadounidense, logró anclar las expectativas inflacionarias y producir una reducción permanente de la tasa de inflación, que fue en promedio para esa década, cercana a cero. La adopción de este esquema de política fue acompañada por una drástica reforma del sector público que incluyó la privatización de la mayor parte de las empresas públicas y la dolarización financiera. El país ganó acceso a los mercados financieros internacionales y con mejores resultados fiscales en un comienzo, el gobierno reemplazó el financiamiento monetario por la emisión de deuda en los mercados internacionales. Esta combinación de políticas fue exitosa en anclar las expectativas inflacionarias y estabilizar la inflación en niveles muy reducidos. Sin embargo, la reforma fiscal fue incompleta. Con la moneda local sobrevaluada, el país comenzó a experimentar persistentes déficits en cuenta corriente, aumentando su endeudamiento externo, tanto privado como público. Luego de las crisis asiática en 1997 y rusa en 1998, el país ingresó en una prolongada recesión, que se acentuó con devaluación del real brasileño en enero de 1999. La mayores tasas de interés sobre la deuda aumentaron el peso de los pagos de intereses en las cuentas públicas y el endeudamiento externo, tanto privado como público, comenzó a ser percibido como insostenible. Hacia 2001 se desencadenó una crisis financiera y externa que derivó en el abandono del régimen de Convertibilidad y una devaluación del peso argentino que implicó un drástico cambio de precios relativos y dió lugar a una aceleración de la inflación, que alcanzó un pico en abril de 2002, para luego reducirse, aunque a un nivel algo más elevado que el prevaleciente bajo el régimen de Convertibilidad. Hacia fines de 2004, cuando la economía comenzó a recuperarse en forma sostenida de la recesión en la que había estado inmersa durante varios años, la inflación comenzó a acelerarse levemente.

\subsection{Análisis descriptivo}

La breve descripción del comportamiento histórico de la inflación en Argentina en la sección precedente, sugiere la presencia de importante quiebres estructurales y episodios atípicos como puede ser una hiperinflación. 
Aún a pesar de estas especificidades, es razonable esperar que los shocks sobre esa variable no tengan un efecto permanente, en la medida que la política económica en general y la monetaria en particular, actúan en general proveyendo a la economía con algún ancla nominal que logra estabilizar la inflación. En ese sentido, se espera que los tests de raíces unitarias rechacen la hipótesis nula de una raíz unitaria para la inflación cuando se estudian sus propiedades temporales para un período de tiempo suficientemente prolongado. Sin embargo, como se enfatiza en la sección 2, es posible que la tasa media de inflación experimente cambios a través de períodos de tiempo prolongados, si es que las economías experimentan cambios de régimen. En ese caso, se podría esperar no estacionariedad para esta variable, pero atribuible a cambios en su valor de largo plazo y no a la presencia de una raíz unitaria.

Estudiamos en esta sección las propiedades de serie de tiempo de la inflación y evaluamos la presencia de quiebres en su valor medio mediante un análisis descriptivo. En la sección que sigue utilizamos distintas técnicas para identificar la presencia de quiebres tanto en la media como en el componente autorregresivo de la inflación, con el propósito de identificarlos y controlar por ellos en el cálculo de medidas de persistencia inflacionaria.

Se puede observar de la Tabla 2 que tanto la media como la volatilidad de la inflación fueron cambiantes a lo largo de la muestra y que en ese sentido los estadísticos descriptivos para el período completo son poco informativos acerca del comportamiento de la inflación entre 1980 y 2007. Es posible identificar un período de alta inflación entre 1980:1 y 1989:3, el episodio hiperinflacionario entre 1989:4 y 1990:3, una transición entre 1990:4 y 1991:2, seguida de un período de desinflación entre 1991:3 y 1992:12, luego de la implementación de la Convertibilidad.

Tabla 2

\begin{tabular}{lcc}
\multicolumn{3}{c}{ Inflación mensual } \\
medias y desvíos estándar \\
media & desvío estándar \\
1980:1-1989:3 & 0.1034 & 0.0616 \\
$1989: 4-1990: 3$ & 0.4430 & 0.2999 \\
$1990: 4-1991: 2$ & 0.1136 & 0.0512 \\
$1991: 3-1992: 12$ & 0.0517 & 0.0556 \\
$1993: 1-2007: 2$ & 0.0045 & 0.0105 \\
$2002: 1-2002: 9$ & 0.0371 & 0.0232 \\
$2002: 10-2007: 2$ & 0.0062 & 0.0041 \\
$1980: 1-2007: 2$ & 0.0589 & 0.1125
\end{tabular}

El resto de la muestra, que cubre el período 1993:1-2007:2 aparece, desde un punto de vista estrictamente estadístico, como un período de baja inflación, brevemente 
interrumpido por un episodio inflacionario luego de la devaluación del peso en enero de 2002. Como se puede apreciar del Gráfico 1 en la sección anterior, el salto en la inflación provocado por la devaluación de enero de 2002 resulta insignificante si se lo compara con la hiperinflación. Sin embargo hubo en este período un cambio de régimen monetario cuya incidencia en la dinámica inflacionaria no logra ser capturada por el análisis descriptivo. Se intentará estudiarla con más detalle en las siguientes secciones.

Con el objetivo de estudiar las características temporales de la inflación y comenzar a evaluar la presencia de quiebres en su valor medio se estimaron estadísticos $\mathrm{F}$ de Dickey-Fuller que evalúan la presencia de una raíz unitaria, controlando por la significatividad de la media y una tendencia determinística (ver Tabla 3). Un primer resultado es que en todos los casos se rechaza la presencia de una raíz unitaria. Como se deduce de la tabla los test F confirman la ausencia de una media constante a través del período analizado. Entre 1980:1 y 1989:3 la media de la inflación es significativamente distinta de 0 (positiva). Por el contrario, no se rechaza la nula de una media igual a cero entre enero 1993 y 2007. Por otro lado, no se identifica una tendencia determinística estadísticamente significativa en ninguno de los dos períodos, pero sí al considerar el período completo.

Tabla 3

\begin{tabular}{|c|c|c|c|}
\hline \multicolumn{4}{|c|}{$\begin{array}{l}\text { Inflación mensual } \\
\text { Estadístico F de Dickey Fuller }\end{array}$} \\
\hline & Constante & Tendencia & $\mathrm{H}_{0}=$ raíz unitaria \\
\hline 1980:1-1989:3 & Significativa*** & No significativa & Rechazada** \\
\hline 1993:1-2007:2 & No significativa & No significativa & Rechazada** \\
\hline 1980:1-2007:2 & Significantiva ${ }^{* \star *}$ & Significativa*** & Rechazada*** \\
\hline
\end{tabular}

Estos resultados sugieren que si bien la inflación no tiene una raíz unitaria, tampoco puede ser considerada como un proceso estacionario, en la medida que se identifican cambios significativos en su valor medio. En la sección que sigue complementamos el análisis descriptivo con tests que evalúan la presencia de quiebres estructurales tanto en la media como en el componente autorregresivo de la tasa de inflación.

\subsection{Identificando quiebres en la tasa media de inflación}

En esta sección se busca identificar la presencia de quiebres tanto en la media como en el componente autorregresivo de la inflación y estudiar su relación con la ocurrencia de cambios en el régimen monetario. 


\subsubsection{Análisis recursivo}

Comenzamos por estimar recursivamente um modelo autorregresivo para la inflación, de acuerdo a la ecuación 12 y evaluar la presencia de quiebres tanto la constante como en el coeficiente autorregresivo, utilizando tests de cambio estructural. El gráfico 6.a muestra que ambos coeficientes, constante y término autorregresivo se ubican fuera del intervalo de $+/$ - 2 veces los desvíos estándar previos en el entorno de la hiperinflación. Los tests de Chow, en el gráfico 6.b. ("forecast horizon" descendent, ascendant and one-step), se ubican debajo del valor crítico de $5 \%$ excepto en el período de la hiperinflación.

$$
\pi_{t}=\alpha+\sum_{i=1}^{p} \beta_{i} \pi_{t-i}+\eta_{t}
$$


Gráficos 6.a. y 6.b. Análisis recursivo (1980:1-2007:2)
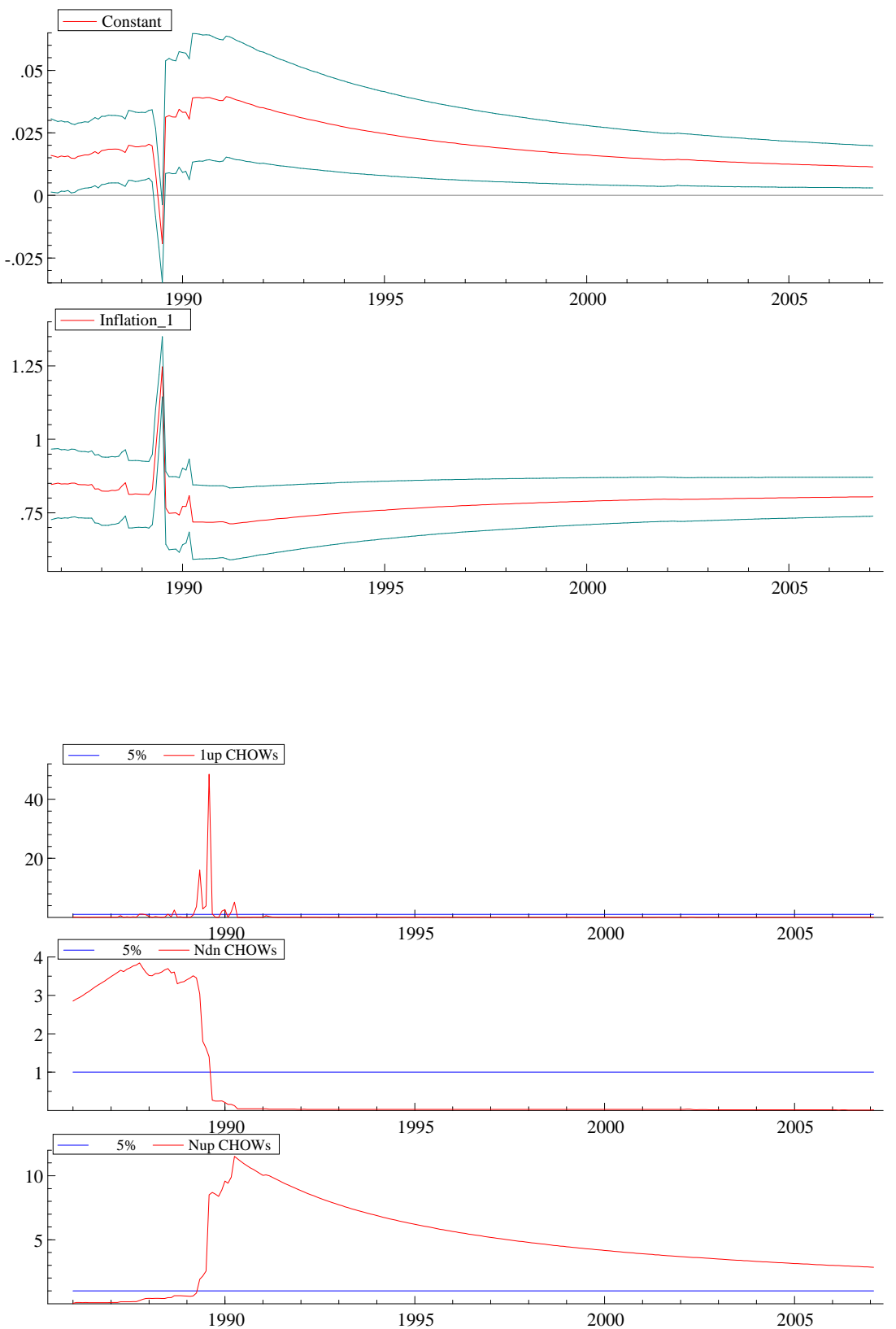

En resumen, se encuentra evidencia de un cambio en la dinámica de la inflación en el entorno del episodio hiperinflacionario, que finalizó con la adopción de un nuevo régimen cambiario, la Convertibilidad. Debido probablemente a la magnitud del episodio hiperinflacionario, y a la volatilidad generada por el mismo, no es posible identificar un quiebre significativo en la dinámica de la inflación luego del abandono de ese régimen. Es por esa razón que más adelante estudiamos ese subperíodo en forma separada. 


\subsubsection{Test de Bai Perron}

La otra aproximación que llevamos adelante para estudiar la presencia de quiebres estructurales en el proceso dinámico de la inflación es la implementación de los tests desarrollados por Bai y Perron (2003) para evaluar la presencia de múltiples quiebres estructurales. En relación al análisis precedente, la metodología de Bai y Perron es más general. Permite identificar la presencia de múltiples quiebres, y propone tests para evaluar la hipótesis nula de no quiebres vs. la presencia de múltiples quiebres, asi como un procedimiento para evaluar la hipótesis nula de $n$ quiebres vs. $n+1$ quiebres. También genera intervalos de confianza para las fechas de quiebre, permitiendo que los datos y los errores tengan diferentes distribuciones entre los segmentos en los que el test separa la muestra, o eventualmente impone una distribución común. Esta flexibilidad es interesante en el caso argentino dada la presunción de una varianza no constante a través del período estudiado.

Llevamos adelante el test bajo dos especificaciones. La primera supone la presencia de quiebres en la media, mientras que la segunda permite cambios tanto en la media como en el componente autorregresivo de la inflación. Los resultados de ambos ejercicios se muestran en la Tabla 4 . 


\section{Tabla 4}

\begin{tabular}{|c|c|c|c|c|}
\hline$z_{t}=1$ & $q=1$ & $\begin{array}{c}\mathrm{p}=0 \\
\text { Tests }\end{array}$ & $h=81$ & $M=1$ \\
\hline $\operatorname{SupF}_{\mathrm{T}}(1)$ & UDmax & WDmax & $\operatorname{SupF}_{\mathrm{T}}(2 \mid 1)$ & \\
\hline \multicolumn{5}{|c|}{ Número de quiebres seleccionado } \\
\hline $\begin{array}{c}\text { Sequential } \\
1\end{array}$ & $\begin{array}{c}\text { BIC } \\
1\end{array}$ & $\begin{array}{c}\mathrm{LWZ} \\
1 \\
\text { timacio }\end{array}$ & & \\
\hline alfa & alfa & $\mathrm{T}_{1}$ & & \\
\hline $\begin{array}{c}0.135 \\
(0.0336)\end{array}$ & $\begin{array}{c}0.006 \\
(0.0016)\end{array}$ & Apr-91 & & \\
\hline
\end{tabular}

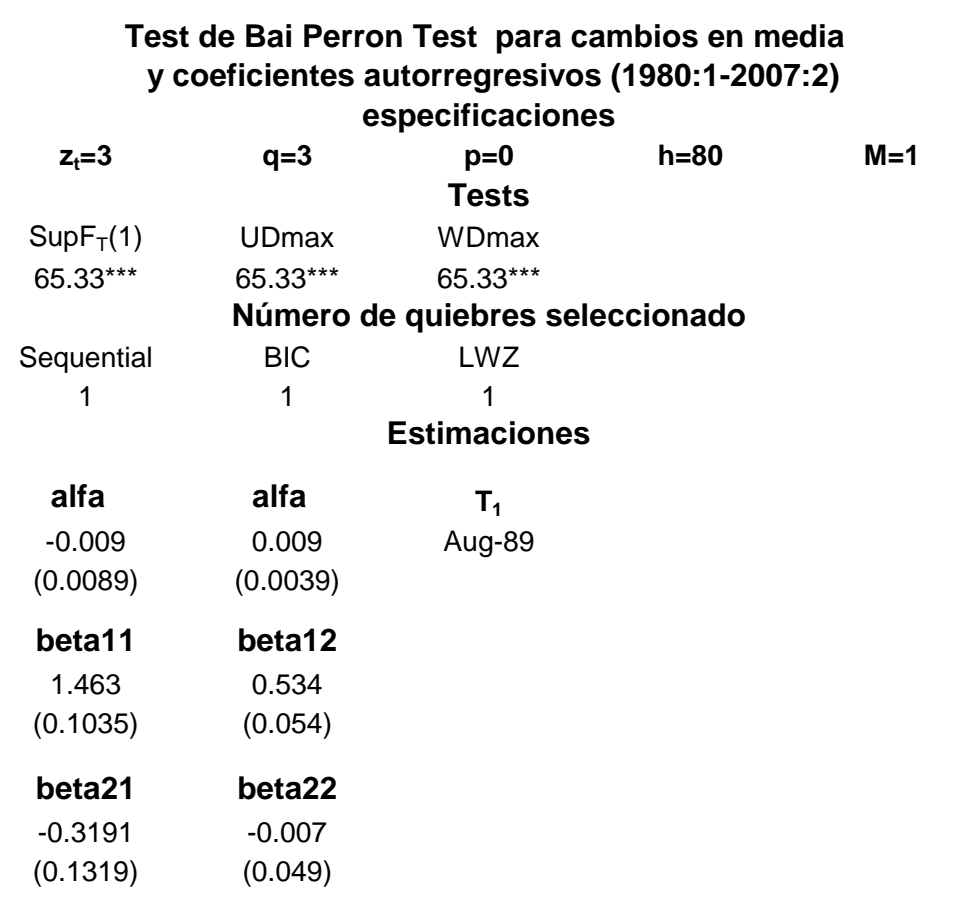

En el primer caso comenzamos considerando la posibilidad de hasta 5 quiebres en la media, pero sólo uno resulta significativo de acuerdo a los criterios propuestos por Bai y Perron: SupF Sequential Procedure, Bayesian Information Criterion (BIC) and Liu, Wu and Zidek (LWZ) y corresponde a abril de 1991, fecha en que se implementó del plan de Convertibilidad. Al restringir el número de quiebres se vuelve a identificar un único quiebre en abril de 1991, por lo que se reportan en la tabla los resultados correspondientes al test permitiendo un quiebre. En este caso se calcularon los intervalos de confianza para los quiebres permitiendo heteroscedasticidad y correlación serial en los residuos.

En el segundo caso se evalúa la presencia de quiebres tanto en la media como en 
el componente autorregresivo de las serie. Debido a que en este caso se trata de un modelo dinámico, no es posible, de acuerdo a lo sugerido por Bai y Perron permitir heteroscedasticidad y correlación serial en los residuos, no pudiéndose utilizar la matriz HAC propuesta por Andrews (1991). ${ }^{10}$ Cuando evaluamos sólo cambios en la media, si bien se calcula inicialmente el test permitiendo hasta cinco quiebres, este identifica un quiebre en agosto de 1989, fecha que se corresponde con la ocurrencia del primer episodio hiperinflacionario, en forma coincidente con los resultados del análisis recursivo de la subsección precedente. Esta fecha de quiebre lleva a que ambos subperíodos incluyan observaciones extremas y alta volatilidad en la tasa de inflación. Esa puede ser la razón por la que los valores medios que se obtienen para ambos sub-períodos sean poco razonables, lo que no ocurre con los términos autorregresivos, cuyos valores se corresponden con lo esperado: están cerca de sumar 1 para el período de alta inflación, indicando que era un proceso altamente persistente y se reducen significativamente (a 0.53) en el segundo subperíodo.

\subsection{Persistencia inflacionaria}

\subsubsection{Las medidas convencionales de persistencia inflacionaria}

El concepto de persistencia inflacionaria tiene cierta especificidad en el contexto de la economía monetaria. En general y desde un punto de vista estrictamente estadístico la persistencia es una medida de la velocidad con que una variable retorna a su valor medio. En el marco de la economía monetaria, ella se refiere a la velocidad con que la inflación retorna a su valor de equilibrio de largo plazo luego de un shock. Ese valor de equilibrio de largo plazo es en alguna medida un reflejo del accionar de la política monetaria en su objetivo de proveer a la economía de un ancla nominal y en ese sentido no es necesariamente único, sino más bien dependiente del régimen monetario vigente.

Una medida ampliamente utilizada de persistencia es la propuesta por Andrews y Chen (1994).

Considerando a la inflación como un proceso estacionario $\operatorname{AR}(\mathrm{p})$

$$
\pi_{t}=\alpha+\sum_{i=1}^{p} \beta_{i} \pi_{t-i}+\eta_{t}
$$

La persistencia se define como la suma de los coeficientes autorregresivos en (12')

\footnotetext{
${ }^{10}$ Debido a que se tiene la presunción de que la presencia de heteroscedasticidad es bastante probable, se realizó el ejercicio de calcular el test bajo ambas especificaciones: utilizando el estimador HAC y el convencional, que supone homoscedasticidad y ausencia de correlación serial en los residuos. Se encontró que los modelos estimados bajo ambos supuestos no difieren significativamente.
} 


$$
\rho=\sum \beta_{i}
$$

y

$$
\mu=\frac{\alpha}{1-\rho}
$$

es la media no condicional de la inflación. Cuanto más elevado es el valor absoluto de $\rho$, más lentamente la inflación retorna a su valor de equilibrio.

Como señalamos arriba, el concepto de persistencia se asocia a la velocidad con que la inflación retorna a su valor de largo plazo luego de un shock. En ese sentido una representación adecuada de ese proceso, como enfatiza Marques (2004) es reescribir (12') en términos de desvíos de su valor de largo plazo.

$$
\pi_{t}-\mu=\sum_{i=1} \beta\left(\pi_{t-i}-\mu\right)+\eta_{t}
$$

que también puede escribirse del siguiente modo

$$
\pi_{t}-\mu=\sum_{i=1}^{p-1} \varphi_{i} \Delta\left(\pi_{t-i}-\mu\right)+\rho\left(\pi_{t-1}-\mu\right)+\eta_{t}
$$

Una cuestión crucial a determinar previo al cálculo de alguna medida de persistencia, es si es adecuado suponer un valor de largo plazo constante para la inflación.

\subsubsection{Persistencia y cambios en la media de la inflación en Argentina}

Tomando en cuenta el análisis descriptivo desarrollado y considerando los quiebres identificados tanto en la tasa media de inflación como en su componente autorregresivo, construimos una media no constante para la tasa de inflación que evoluciona a saltos discretos para luego estimar un modelo autorregresivo de acuerdo a la ecuación 15. Seguimos aqui a Marques (2004) y utilizamos variables dummy que identifican cambios en la tasa media de inflación de acuerdo los quiebres identificados en sección precedente. De ese modo, los valores estimados de la inflación de acuerdo a la ecuación 16 representan la tasa media de inflación que se muestra en el Gráfico 6. En esta ecuación $d 1$ corresponde a una variable dummy para el período 1980:1 -1989:3, $d 2$ para 1989:7-1990:3, $d 3$ para 1990:4-1991:2, $d 4$ para 1991:3-2007:2 y $d 5$ para 2002:12002:8.

$$
\begin{aligned}
& \underset{(H C S E)}{\pi_{t}}=\underset{(0,11)}{0,5434} \underset{(011)}{0,43999} d 1 \underset{(0,15)}{0,1338} d 2-\underset{(0,12)}{0,4298} d 3 \\
& -0,5385 d 4+0,03522 d 5 \\
& (0,11) \quad(0,008)
\end{aligned}
$$


Gráfico 6: Tasa de inflación y media estimada de acuerdo a la ecuación 16

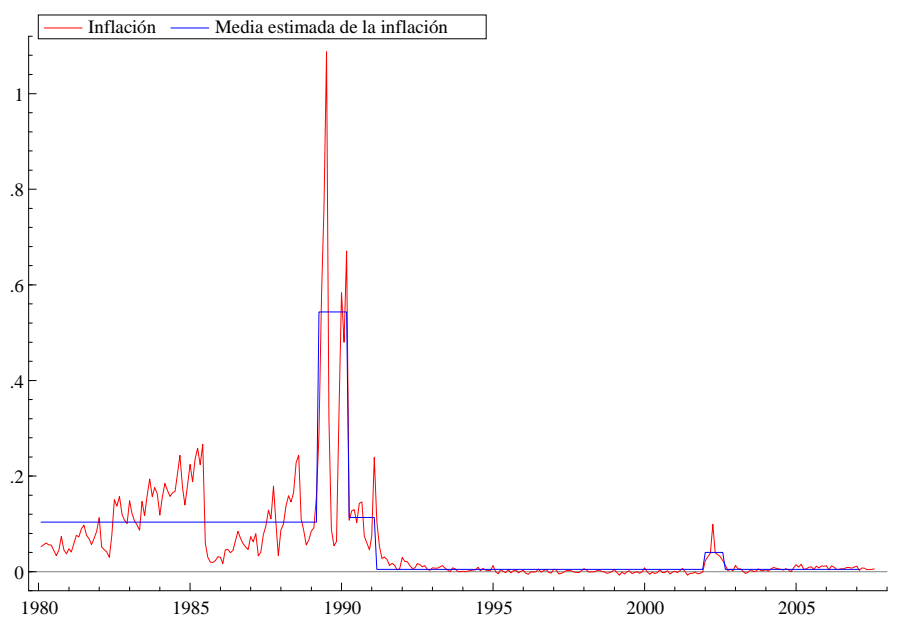

El valor de la constante ( $54.3 \%$ mensual) en la ecuación 16 corresponde a la media de la tasa de inflación en la hiperinflación. De acuerdo esa ecuación y a tests de restricciones lineales, se obtiene una inflación media positiva y distinta de cero $(10.3 \%$, mensual) entre enero de 1980 y marzo de 1989, que resulta de la diferencia entre $d 1$ y la constante. En el período de transición entre abril de 1990 y febrero de 1991 la tasa media de inflación se mantuvo en torno a $11.4 \%$ (su valor se obtiene por diferencia entre $d 3$ y la constante). Luego de la adopción del esquema monetario de la Convertibilidad, la tasa de inflación se redujo marcadamente, resultando estadísticamente no distinta de cero hasta el fin de la muestra, lo que surge de la diferencia entre $d 4 \mathrm{y}$ la constante. Sin embargo, durante este último período, y luego del abandono de la Convertibilidad en enero de 2002, se produjo una aceleración inflacionaria después de la abrupta devaluación del peso (la tasa media de inflación en el subperíodo 2002:12002:8 surge de sumar $d 4, d 5$ y la constante y es aproximadamente $3 \%$ mensual). La inflación alcanzó un pico de $10 \%$ mensual en abril de 2002, para luego desacelerarse. Este rebrote inflacionario resulta, sin embargo de pequeña dimensión en relación al hiperinflacionario. Cuando se evalúa la restricción lineal de una media diferente entre enero y agosto de 2002 con respecto al período 1991-2007 se está cerca de rechazar la nula de medias iguales, lo que sugiere que este corto período de transición puede considerarse como un outlier dentro del período de inflación baja y en ese sentido resulta razonable controlar por su presencia, ya que se trata de un episodio transitorio. En la sección 6.3 se estudia separadamente y en detalle el período de baja inflación, descartando el período de desinflación que siguió a la implementación de la Convertibilidad y al hacerlo es posible detectar cambios en la tasa media de inflación luego de la adopción de la flotación administrada. 
Adicionalmente se evaluó la presencia de una tendencia determinística positiva en el período de alta inflación y una negativa luego de la adopción de la Convertibilidad, que controle por la desinflación, tal como lo sugiere la observación visual. La ecuación 17 incorpora ambas tendencias a la ecuación (16), donde $t 1$ corresponde a una tendencia determinística para el período 1980:1-1989:3 y t2 para 1990:4-1991:2 Ver también el Gráfico 6 .

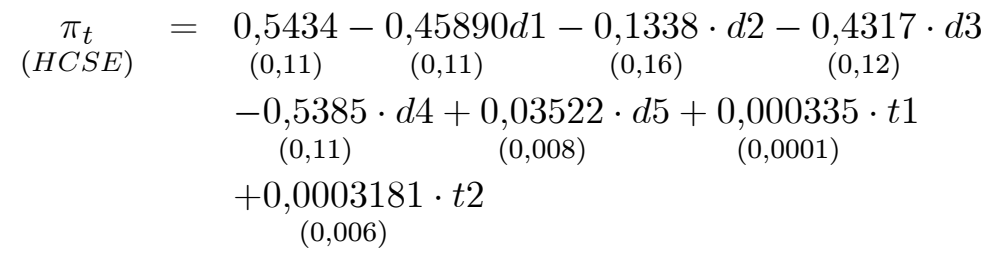

Gráfico 6: Tasa de inflación y media estimada de acuerdo a la ecuación 17

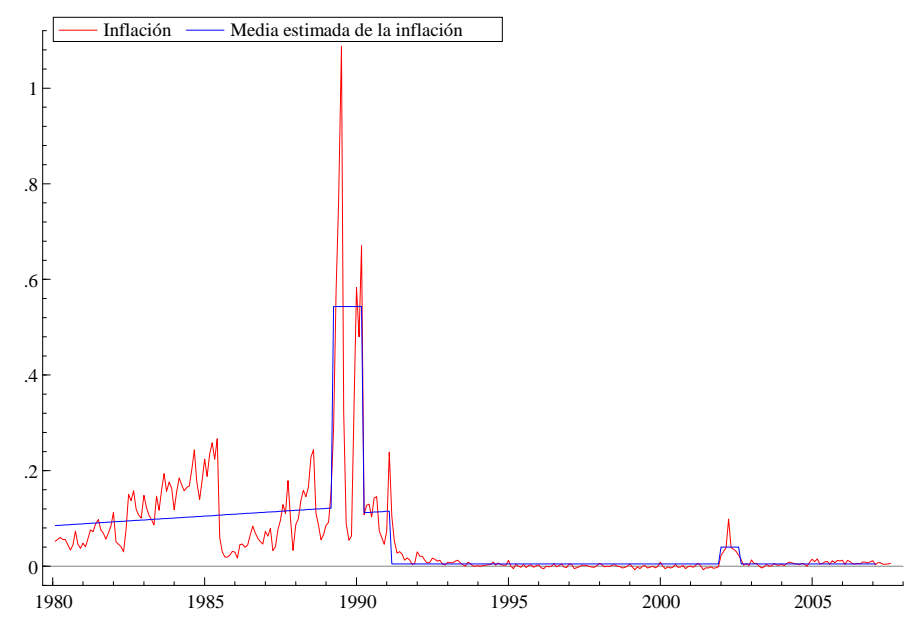

Fuente: INDEC

Habiendo obtenido una media no constante de la tasa de inflación $\left(\mu_{t}\right)$, de acuerdo a la ecuación 17, el siguiente paso es calcular desvíos de la inflación respecto de esa media, a los que llamamos $z_{t}$, para luego estimar la ecuación 14 y calcular una medida de persistencia que considera una media no constante. En la Tabla 5 comparamos la medida de persistencia obtenida a partir de la estimación de la ecuación 14 utilizando una media variable $\left(\mu_{t}\right)$ respecto de la obtenida si se supone una media constante $(\mu)$. 
Tabla 5

\begin{tabular}{lcc}
\multicolumn{3}{c}{ Persistencia de la inflación } \\
Período 1980:1-2007:2 \\
$\rho$ \\
hcse & media constante & cambios en media \\
& 0.80 & 0.56 \\
& $(0.184)$ & $(0.240)$ \\
& $(1 \mathrm{lag})$ & $(1 \mathrm{lag})$
\end{tabular}

Se observa de la Tabla 5 que bajo el supuesto de una media constante, la inflación sería un proceso altamente persistente (0.8). Por el contrario, si permitimos una media variable, el grado de persistencia se reduce notablemente (0.56). Ambas medidas de persistencia son estadísticamente diferentes, lo que nos permite concluir de este ejercicio que una vez que se identifican quiebres en la media de la inflación y se controla por ellos, la inflación aparece como un proceso bastante menos persistente que si se considera una media constante.

Una segunda cuestión a investigar es si, asociado a cambios en la media de la inflación, también se identifican cambios en el componente autorregresivo de la serie. Tanto el análisis recursivo como los tests de Bai Perron presentados en la sección anterior identifican cambios en los coeficientes autorregresivos de la inflación asociados a quiebres en la media de la tasa de inflación. La evidencia reciente para otros países sugiere que una vez que la inflación se reduce, su grado de persistencia es menor. ${ }^{11}$

Con el objetivo de calcular una medida de persistencia que tome en cuenta esos cambios estimamos un modelo autorregresivo de $z_{t}$ (los desvíos de la inflación respecto de su media variables estimada según la ecuación 17 ) incluyendo dummies que multiplican a los niveles y diferencias de $z_{t}$. La ecuación 19 muestra el modelo estimado, donde $d 1$ corresponde a una variable dummy para el período 1980:1-1989, $d 2$ para 1990:4-1991:2, d3 para 1991:3-1992:12 y d4 para 1993:1-2007:12. En la Tabla 6 presentamos las medidas de persistencia obtenidas a partir de esta ecuación.

$$
\begin{aligned}
z_{t}= & 0,5048 z_{t-1}+0,4084 z_{t-1} d 1-0,6708 z_{t-1} d 2+0,3504 \Delta z_{t-1} \\
& {[0,2101] \quad[0,217] } \\
& -0,3063 \Delta z_{t-1} d 1-0,3723 \Delta z_{t-1} d 3-0,4956 \Delta z_{t-1} d 4 \\
& {[0,2284] \quad[0,0737] } \\
& -0,1954 d j u l 95+\text { dummies hiperinflación } \\
& {[0,096] }
\end{aligned}
$$

\footnotetext{
${ }^{11}$ Ver al prespecto Angeloni et al. y Capistrán y Ramos Francia, op. cit.
} 
Tabla 6

\begin{tabular}{cc}
\hline \multicolumn{2}{c}{$\begin{array}{c}\text { Persistencia de la inflación } \\
\text { Period 1980:1-2007:2 }\end{array}$} \\
\hline Sub-períodos & rho \\
1980:1-1989:3 & 0.954 \\
1989:4-1990:3 & 0.855 \\
$1990: 4-1991: 2$ & 0.184 \\
1991:3-1992:12 & 0.483 \\
$1993: 1-2007: 2$ & 0.359 \\
\hline
\end{tabular}

Los resultados indican que la inflación fue un proceso altamente persistente durante el período de alta inflación (0.96), entre 1980:1 y 1989:3, más aún que cuando se supone una media constante para toda la muestra. Los períodos subsecuentes en la Tabla 6 corresponden a la hiperinflación (1989:4-1990:3), la transición (1990:4-1991:2) y la desinflación (1991:3 - 1992:12) que siguió a la implementación del régimen de Convertibilidad. Si bien no estamos interesados en medir el grado de persistencia de la inflación en esos períodos, debimos controlar por ellos en la estimación para medir adecuadamente la persistencia en los períodos relevantes. Durante el período que aqui denominamos de baja inflación, el grado de persistencia se reduce marcadamente, a 0.36 .

En resumen, el análisis precendente indica que hubo cambios significativos tanto en la media de la inflación como en su dinámica autorregresiva durante el período que analizamos. Controlando por esos cambios se encuentra que la inflación fue un proceso altamente persistente en el período de inflación elevada (cercano a un camino aleatorio) y su persistencia se redujo marcadamente una vez que ésta se estabilizó a niveles promedio significativamente más bajos, luego del episodio hiperiflacionario que Argentina experimentó a fines de los 80's. También se encuentra evidencia de que los cambios tanto en la media como en el componente dinámico están relacionados con el cambio en el régimen monetario que implicó la adopción de un régimen tan peculiar como la Convertibilidad. El hecho de que la dinámica inflacionaria se acercara al comportamiento de un camino aleatorio durante el período de inflación alta parece consistente con las dificultades que encontró la política económica durante ese período para anclar las expectativas inflacionarias, haciendo retornar a la inflación hacia algún valor de equilibrio de largo plazo. El pasaje por un episodio tan traumático como la hiperinflación parece haber generado los ajustes necesarios, principalmente fiscales, como para que un anclaje como el de la Convertiblidad lograra estabilizar la tasa de inflación a niveles muy reducidos. El cambio de régimen implicado por el abandono de ese esquema monetario no puede ser captado cuando se incluye un período atípico como la hiperinflación, razón por la que en la sección siguiente estudiamos el subperíodo 1993-2007 en forma separada. 


\subsubsection{Un análisis detallado del período de baja inflación}

Como enfatizamos en la sección anterior, las dramática volatilidad implicada por el episodio hiperinflacionario limita la posibilidad de identificar quiebres adicionales en la serie de inflación y en particular el potencial cambio asociado al abandono de la Convertiblidad y la adopción de un régimen de flotación administrada en 2002. Es por esta razón que en esta sección estudiamos separadamente al período que denominamos de baja inflación, en que convivien dos subperíodos potencialmente distintos en términos de la política monetaria vigente, de cuyas características no parece estar desvinculada la dinámica inflacionaria, de acuerdo al análisis de la sección precedente y a la amplia evidencia empírica que estudia la relación entre el dinero y los precios. ${ }^{12}$

Para estudiar en que medida la adopción de un régimen de flotación administrada en enero de 2002 implicó cambios en la dinámica de la inflación, se analiza separadamente este subperíodo. El análisis recursivo permite identificar un quiebre tanto en la media, como en el término autorregresivo del modelo AR (1) estimado a comienzos de 2002, en forma coincidente con el abandono del régimen de Convertibilidad en enero de ese año. El quiebre en el componente autorregresivo sugiere que el grado de persistencia puede haber cambiado entre ambos regímenes.

\footnotetext{
${ }^{12}$ Ver al respecto McCandless y Weber (1995), De Grawue y Polan (2002) y Gabrielli et al. (2004) y Basco et al. (2006) para el caso argentino.
} 
Gráficos \%.a.y \%.b:Análisis recursivo (1993:1-200\%:2)
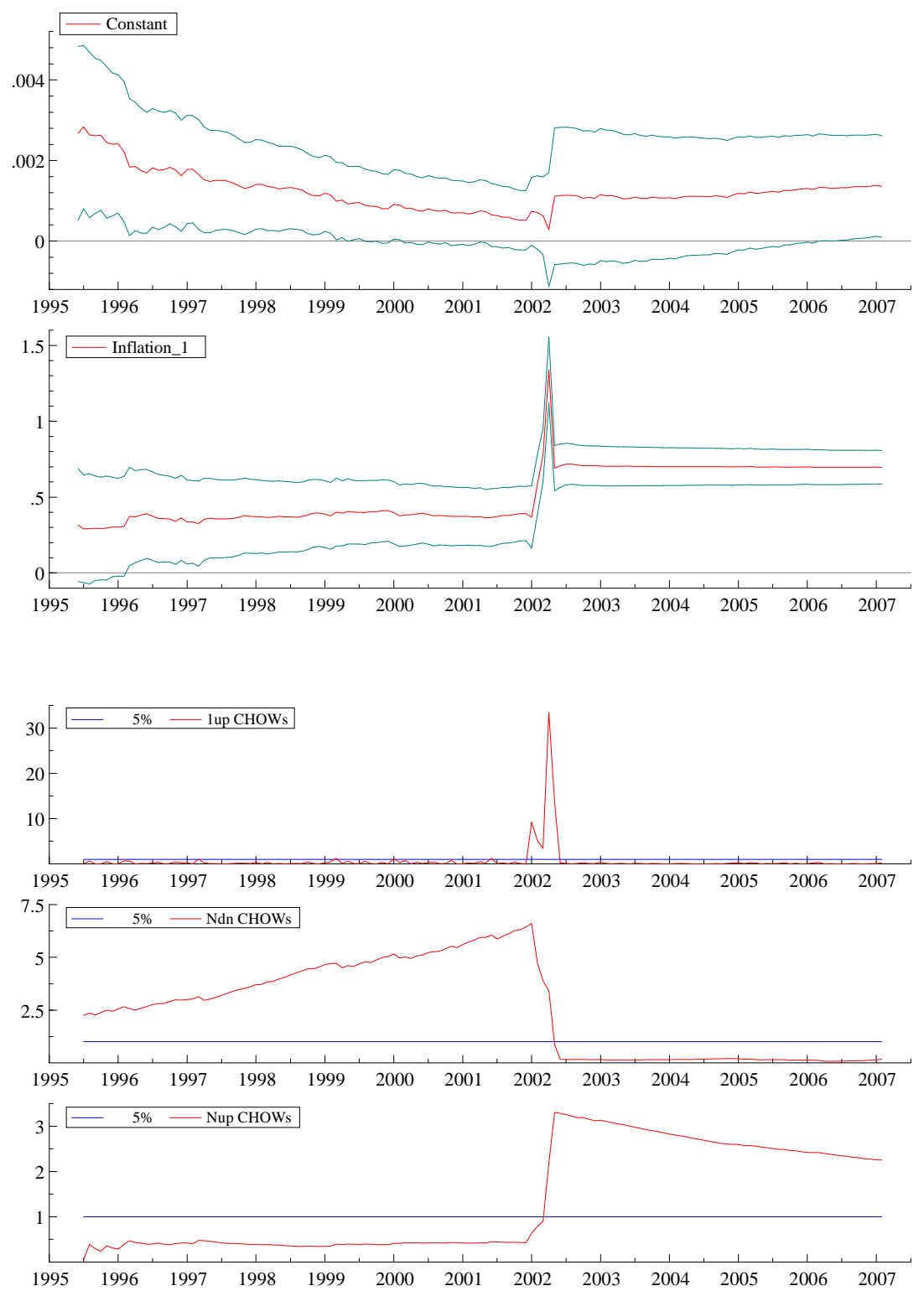

Estos resultados se confirman con el test de Bai Perron (ver Tabla 7), que identifica un quiebre en enero de 2002 cuando testeamos quiebres en la tasa media de inflación y en mayo de ese mismo año cuando evaluamos cambios en la media y en el componente autorregresivo de la serie. 


\section{Tabla 7}

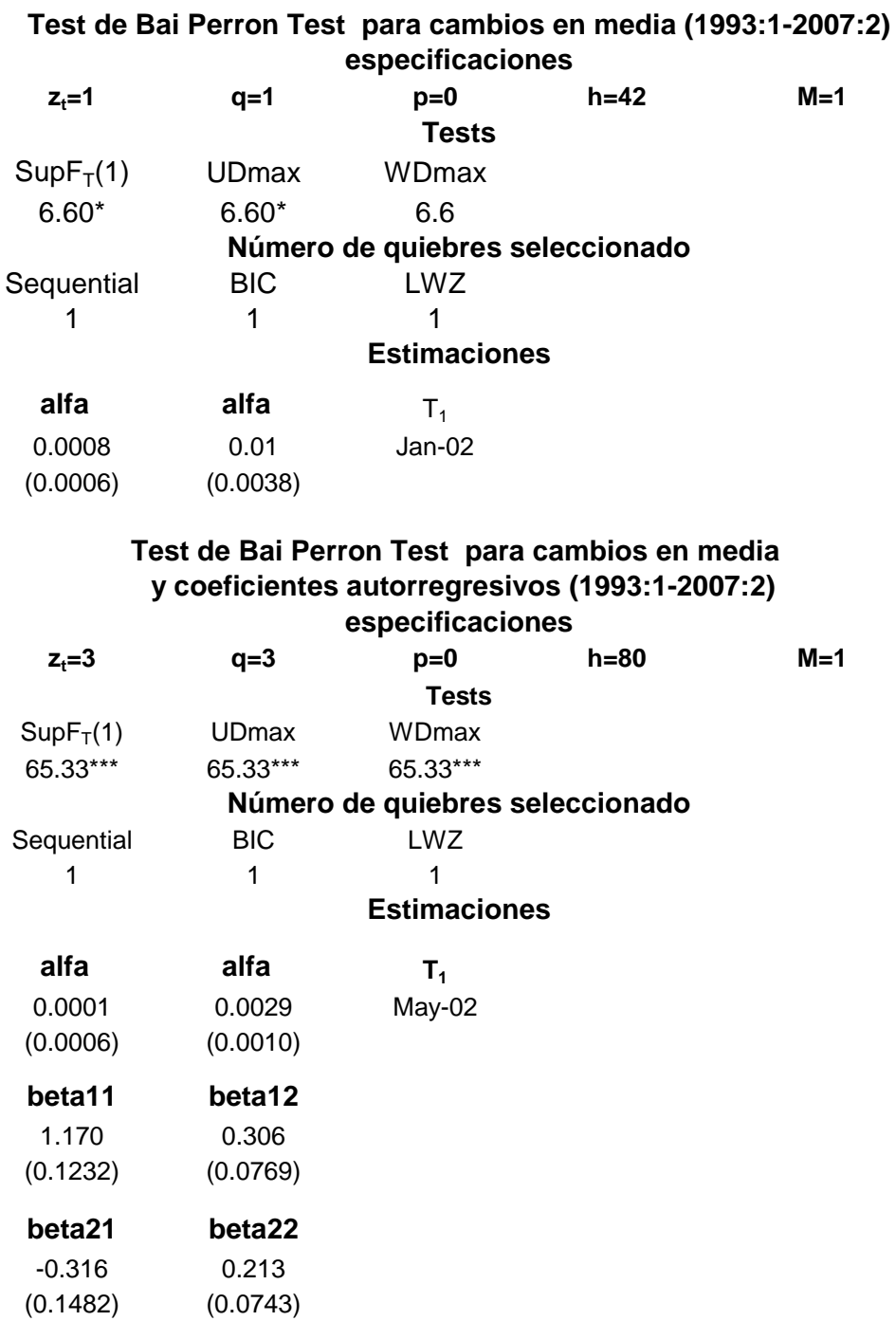

Luego de haber identificado la presencia de al menos un quiebre en la tasa media de inflación, construimos una media variable para ese período. La ecuación 19 muestra los resultados de esa estimació. En esa ecuación $d 1$ corrensponde a una variable dummy para el período 2002:1-2002:9 y d2 para el perído 2002:10-2007:2. El Gráfico 8 muestra la evolución de la tasa de inflación en ese período y la de la media estimada.

$$
\underset{(H C S E)}{\pi}=\underset{(0,0003)}{0,00087}+\underset{(0,0078)}{0,0362} d 1+\underset{(0,0006)}{0,0053 d 2}
$$


Gráfico 8: Inflación y media estimada de la inflación de acuerdo a la ecuación 19

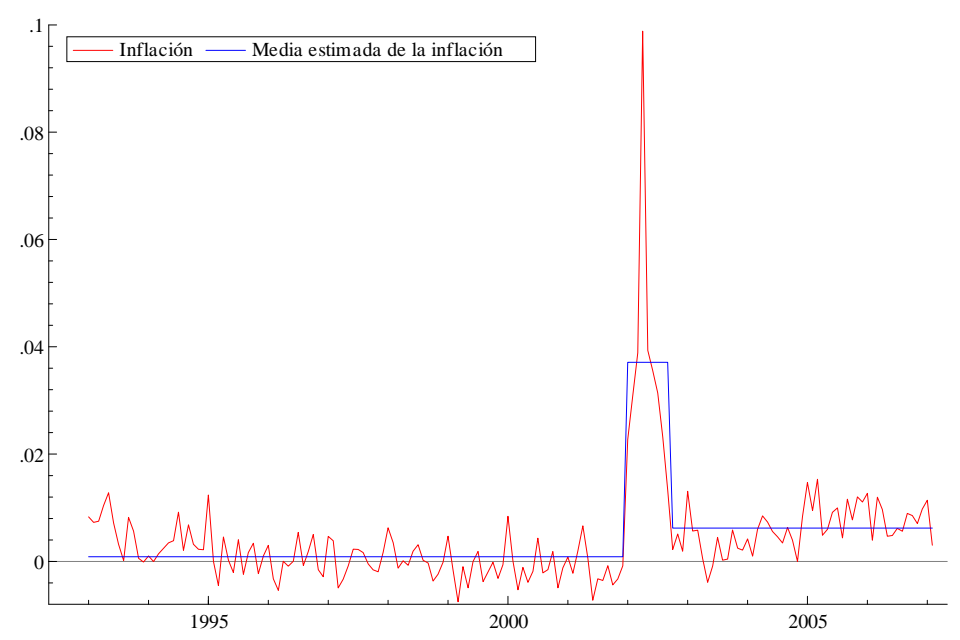

Se observa que la tasa media de inflación fue levemente distinta de 0 durante el período de la Convertibilidad. Luego durante la crisis que siguió a la devaluación de enero de 2002, la tasa media de inflación se elevó a $3.6 \%$ mensual para luego reducirse a una tasa media $0.5 \%$, que resulta positiva y estadísticamente diferente de cero. Se tiene entonces que, una vez que consideramos el período de baja inflación separadamente, es posible identificar un quiebre que aparece asociado al cambio de régimen implicado por el abandono de la Convertiblidad. También se identifica un período atípico en los meses que siguen a la devaluación de enero de 2002, en el que la inflación experimenta un salto transitorio. Controlamos también por ese período al calcular la media de la inflación de acuerdo a la ecuación 19.

En la Tabla 8 mostramos los resultados de calcular medidas de persistencia para el período 1993:1-2007:2 utilizando una media constante y una media variable. Se observa una importante reducción en la persistencia estimada cuando se consideran los quiebres identificados de acuerdo a la ecuación 19 . 
Tabla 8

\begin{tabular}{lcc}
\multicolumn{3}{c}{ Persistencia de la inflación } \\
Período 1993:1-2007:2 \\
$\rho$ \\
hcse & media constante & cambios en media \\
& 0.70 & 0.18 \\
& $(0.207)$ & $(0.082)$ \\
$(1 \mathrm{lag})$ & $(1 \mathrm{lag})$
\end{tabular}

Finalmente, cuando tratamos de identificar la presencia de cambios en los coeficientes autorregresivos de la inflación que podría estar asociados al cambio de régimen, encontramos, de acuerdo a la ecuación 19 un muy bajo grado de persistencia (0.15) durante el período de la Convertibilidad, que se incrementa significativamente (0.27), luego de la adopción de la flotación administrada. En esa ecuación $d 1$ es una variable dummy que corresponde al período 2002:1-2002:7.

$$
\begin{aligned}
& z_{t}=\underset{[0,0688]}{0,1493 z_{t-1}}+\underset{[0,1157]}{0,2636 z_{t-1}} d 3-\underset{[0,0755]}{0,1437 \Delta z_{t-1}} d 3+\underset{[0,0003]}{0,01148 d 951} \\
& \text { +dummies crisis } 2001
\end{aligned}
$$

En resumen, los resultados obtenidos al analizar el período de baja inflación separadamente indican un cambio en el proceso de la inflación tanto en términos de su valor medio como de su componente autorregresivo, observándose un mayor grado de persistencia en el último superíodo de flotación administrada.

\subsection{Conclusiones}

La evidencia empírica reciente ha revelado que la persistencia puede no ser una característica intrínseca a la inflación y que la dinámica inflacionaria puede cambiar dependiendo del régimen monetario que prevalezca en la economía. Estos estudios revelan además, la importancia de considerar la posibilidad que el valor de largo plazo de la inflación experimente quiebres cuando se calculan medidas de persistencia inflacionaria. También muestran que con la reducción de la inflación como un fenómeno bastante extendido entre las economías, su dinámica también parece haber cambiado y en particular la persistencia inflacionaria se ha reducido. 
En el caso argentino es evidente la presencia de quiebres en el valor de largo plazo de la inflación. La inflación fue muy elevada en los años 80 , un período en el que la política monetaria estaba fuertemente condicionada por desequilibrios fiscales persistentes, que implicaban una alta dominancia fiscal, limitando el accionar de la política monetaria. Luego de un episodio hiperinflacionario a fines de esa década, el país adoptó un régimen de caja de conversión que logró estabilizar de manera bastante permanente la tasa de inflación en niveles reducidos. Bajo ese régimen la política monetaria era pasiva y la dinámica inflacionaria estaba principalmente gobernada por factores externos. La Convertibilidad fue abandonada luego de la devaluación del peso a comienzos de 2002, adoptándose un régimen de flotación cambiaria. La devaluación del peso fue seguida de una breve aceleración inflacionaria luego de la cual la inflación volvió a ubicarse en niveles más reducidos, aunque algo más elevados que los que prevalecieron durante la Convertibilidad.

Analizamos la dinámica de la inflación durante este período y en particular su persistencia. Estudiamos las implicancias para la modelación de la política monetaria de suponer que la inflación es un proceso altamente persistente a través de un ejercicio de simulación. Utilizando metódos recursivos y los tests de cambio estructural desarrollados por Bai y Perron identificamos quiebres en la tasa media de inflación que resultan coincidentes con cambios en el régimen monetario: la adopción de la Convertiblidad en 1991 y el abandono de ese régimen en enero de 2002, cuando consideramos en período de baja inflación separadamente. Dada la presencia de cambios en la tasa media de inflación, diferenciamos esta variable respecto de esa media que evoluciona según quiebres discretos y calculamos medidas de persistencia inflacionaria. Encontramos que la inflación fue un proceso altamente persistente durante el período de alta inflación, cercano a un camino aleatorio. Por el contario, con el descenso de la inflación a partir de la adopción del régimen de Convertibilidad, su persistencia se redujo marcadamente. Luego de la introducción del régimen de flotación administrada y controlando por el episodio inflacionario desatado por la devaluación del peso en 2002, encontramos que la inflación vuelve a mostar un comportamiento más persistente. Estos resultados corroboran la importancia de evaluar la presencia de quiebres estructurales al modelar la dinámica inflacionaria y en particular al intentar estimar su persistencia. También confirman que en el caso argentino la persistencia no es una característica intrínseca de la inflación sino dependiente del régimen monetario. Los resultados obtenidos también indican que la persistencia inflacionaria no es muy elevada actualmente, lo que sugiere que los costos de desinflar la economía tampoco deberían serlo. 


\section{Bibliografía}

[1] Altissimo, F., B. Mojon y P. Zaffaroni (2006), "Sectoral and Aggregate Inflation Dynamics in the Euro Area". Journal of the European Economic Association, AprilMay 2006, Vol. 4, No. 2-3, Pages 585-593.

[2] Altissimo, F., B. Mojon y P Zaffaroni (2004), "Fast macro and slow micro: Can aggregation explain the persistence of inflation?", FRB of Chicago Working Paper No. 2007-02.

[3] Andrews, D.W.K. y H-Y Chen (1994), . ${ }^{\mathrm{A}}$ pproximately Median -ubiased Estimation of Autorregresive Models", Journal of Business and Economic Statistics, 12(2) 187204.

[4] Angeloni, I., L. Aucremanne, M. Ehrmann, J. Galí, A. Levin y F. Smets (2006), "New Evidence on Inflation Persistence and Price Stickiness in the Euro Area: Implications for Macro Modeling". Journal of the European Economic Association, April-May 2006 4(2-3):562-574.

[5] Ascari, G. y T. Ropele (2007). "Trend Inflation, Tayor Principle and Indeterminancy", Kiel Working Paper $N^{\circ}$ 1332. Kiel Institute for World Economics.

[6] Bai, J. y P. Perron. (2003), "Computation and Analysis of Multiple Structural Change Models", Journal of Applied Econometrics, 18, 1, 1-22.

[7] Basco, E. L. D 'Amato y L.Garegnani, (2006), "Understanding the money - prices relationship under low and high inflation regimes: Argentina 1977 - 2006", Documento de Trabajo 2006/11 BCRA.

[8] Blake A. y E. Fernández-Corugedo (2006), "Optimal monetary policy with nonzero steady-state inflation", Mimeo.

[9] Cagan, R. (1956) "The Monetary Dynamics of Hyperinflation", in M. Friedman ed., Studies in the Quantity Theory of Money, Chicago: University of Chicago Press. 
[10] Calvo, G., (1983), "Staggered contracts in a utility maximizing framework", Journal of Monetary Economics, 12, 383-398.

[11] Capistrán, C. y M. Ramos-Francia (2006), "Inflation Dynamics in Latin America" Banco de Mexico, WP No 2006-11.

[12] Castillo, P., A. Humala y V. Tuesta (2006), "Monetary Policy, Regime Shifts and Inflation Uncertainty in Peru (1949-2006)". Mimeo.

[13] De Grauwe, P. y M. Polan (2001), "Is Inflation Always and Everywhere a Monetary Phenomenon?". Discussion Paper $\mathrm{N}^{\circ} 2841$, CEPR.

[14] Elliott, G., T. J. Rothenberg y J. H. Stock (1996), "Efficient Tests for an Autoregressive Unit Root". Econometrica 64, 813-836.

[15] Elosegui P., G Escudé, L. Garegnani y J.M. Sotes Paladino (2007), "El Modelo Económico Pequeño del BCRA", Documento de Trabajo 2007/18, BCRA.

[16] Erceg, C y A. Levin (2003), "Imperfect Credibility and Inflation Persistence", Journal of Monetary Economics, 50, 915-944.

[17] Fuhrer, J.. y G. Moore (1995a), "Inflation Persistence". Quarterly Journal of Economics 110 (1): 127-159.

(1995b), "Monetary Policy Trade-Offs and the Correlation Between Nominal Interest Rates and Real Output". American Economic Review 85 (marzo): 219-239.

(2006), "Intrinsec and Inhereted Inflation Persistence", International Journal of Central Banking, septiembre 2006, Vol. $2 \mathrm{~N}^{o} 3$.

[18] Gabrielli, F, G. Mc. Candless y M. Rouillet (2004), "The Intertemporal Relation Between Money and Prices. Evidence from Argentina", Cuadernos de Economía, Vol. 41(Agosto), 199-215.

[19] Galí, J. y M. Gertler (1999), "Inflation Dynamics: A structural econometric analysis", Journal of Monetary Economics, vol 4, p. 195-222.

[20] Hamilton, J. D. (1994), "Time Series Analysis" Princeton University Press.

[21] Heymann, D.y A. Leijonhufvud (1995), "High Inflation". Clarendon Press, Oxford.

[22] Heymann, D. y F. Navajas (1990), "Conflicto distributivo y déficit fiscal. Notas sobre la experiencia argentina", en "Inflación Rebelde en América Latina", Arellano J. (compilador), CIEPLAN -Hacchete. 
[23] Kiley, M. (2007), "Is Moderate-to-high Inflation Inherently Unestable?", International Journal of Central Banking, Vo. $3 \mathrm{~N}^{\circ} 2$.

[24] Levin, A. y J. Pigier (2004), "Is Inflation Persistence Intrinsic in Industrial Economies?". $E C B, W P N^{\circ} 334$.

[25] Marques, R. (2004), "Inflation Persistence: Facts or Artifacts?". ECB WP No 371.

[26] Mc Candless G. y W. Weber, (1995), "Some Monetary Facts", Quarterly Review, 19:1173-1193, Federal Reserve Bank of Minneapolis.

[27] Roberts, J., (1997), "Is inflation sticky?", Journal of Monetary Economics, 39 179-196.

[28] Sargent, T. J., W. Noah y T. A. Zha (2006), "The Conquest of South American Inflation". NBER Working Paper No. W12606.

[29] Taylor, J.B., (1979), "Staggered contracts in a macro model", American Economic Review, 69, 108-113.

[30] Taylor, J.B., (1980), "Aggregate dynamics and staggered contracts", Journal of Political Economy 88, 1-23.

[31] Walsh, C., (2003), "Monetary Theory and Policy", MIT Press, Cambridge, Massachsetts. 


\section{Capítulo 3}

\section{Pronóstico de inflación utilizando múltiples predictores: modelos causales y modelos a-teóricos}

\subsection{Motivación ${ }^{1}$}

El pronóstico de la inflación juega un papel central en la formulación de la política monetaria. Al mismo tiempo la evidencia empírica internacional reciente sugiere que con el descenso de la inflación en los últimos años, como un fenómeno bastante extendido, la dinámica conjunta de esta variable y sus potenciales predictores, como el dinero o distintas medidas del grado utilización de los recursos, ha cambiado y la inflación se ha tornado más impredecible. Los modelos univariados suelen superar en capacidad de pronóstico a los basados en las distintas teorías de la inflación como la curva de Phillips. Tradicionalmente la curva de Phillips desempeñó en los países industriales un papel predominante en el pronóstico de inflación y como señalan Stock y Watson (1999), Atkenson y Ohanian (2001) y Canova, (2001) parecía tener mejor desempeño en términos de su error de pronóstico que distintos modelos alternativos. En años recientes ha surgido envidencia, particularmente para los Estados Unidos, de que la curva de Phillips se tornó inestable partir de la decáda de los 80 y también, quizás debido a ello, su capacidad de pronóstico se debilitó, siendo superada en general por los modelos univariados.

Clements y Hendry (2006) sugieren que esta dificultad de los modelos causales puede estar asociada a la presencia de cambios de régimen que afectan principalmente

\footnotetext{
${ }^{1}$ Agradecemos las valiosas sugerencias de Hildegart Ahumada, Carlos Capistrán, Bárbara Rossi y Kenneth West, asi como también los comentarios recibidos en el Seminario en el Banco Central de Chile, en octubre de 2008 y en la XLII Reunión de la AAEP en noviembre de 2008.
} 
los componentes determinísticos de los modelos y proponen una estrategia de modelación basada en una batería de modelos que permiten lidiar con esa dificultad. Otra estrategia propuesta por la literatura de pronóstico es trabajar con combinaciones o pooling de pronósticos, teoría desarrollada inicialmente por Bates y Granger (1969). Hendry y Clements (2002) estudian el pooling o combinación de pronósticos para modelos no estacionarios debido a quiebres en la media o en una tendencia determinística, y encuentran que un promedio simple de pronósticos puede contrarrestar, para valores plausibles de los parámetros, la inestabilidad de los pronósticos individuales y actuar como una corrección sobre el intercepto. Una estrategia alternativa es utilizar modelos que incorporen un número muy grande de predictores, como proponen Stock y Watson (1999, 2006) entre otros, mediante el uso de técnicas estadísticas que permiten construir medidas resumidas (factores) de la variabilidad de un gran conjunto de series económicas.

En este capítulo no concentramos en la evaluación del desempeño de distintos modelos de pronóstico de inflación para Argentina, algunos de los cuales hacen uso de estas técnicas de pronóstico. También comparamos su desempeño relativo utilizando un conjunto de tests propuestos por Diebold y Mariano (1996). No encontramos evidencia de trabajos anteriores que hayan desarrollado modelos de pronóstico de inflación en Argentina y comparado su desempeño relativo utilizando tests estadísticos. Utilizando como benchmark un modelo univariado, evaluamos en este capítulo la capacidad predictiva de algunos modelos causales asociados a distintas teorías de la inflación, como la curva de Phillips y un $V A R$ monetario. Estudiamos también la capacidad predictiva de modelos que utilizan como predictores factores que resumen la variabilidad conjunta de un gran número de series del ciclo económico.

Habiendo identificado en el capítulo anterior quiebres estructurales en la inflación, nos enfocamos aqui en la búsqueda de modelos que tengan buen desempeño para pronóstico. Con ese objetivo restringimos el análisis al período 1993-2006, descartando el período de inflación elevada y el episodio hiperinflacionario de 1989-1990. Evaluamos los modelos considerados out of sample para distintos horizontes de pronóstico relevantes para la política monetaria: un año, que suele ser el horizonte para el que se fija la meta de la política monetaria, ya sea sobre la tasa de inflación o como en Argentina en la actualidad, sobre la expansión de un agregado monetario relevante. También se evaluamos la capacidad de pronóstico de los modelos en horizontes más cortos: 6 y 3 meses, que también tiene relevancia para el seguimiento del ciclo y la toma de decisiones. 


\subsection{Los modelos de pronóstico en la literatura}

Los modelos econométricos causales ofrecen muchas veces una representación satisfactoria del proceso generador de los datos en términos de los comportamientos sugeridos por la teoría económica. Sin embargo estos modelos suelen tener pobre desempeño en el pronóstico de series de tiempo relevantes, cuando se lo compara con el de los modelos autorregresivos. Una razón para ello es que estos últimos parecen ajustar mejor a cambios no anticipados en el proceso generador de los datos, por su carácter adaptativo.

En los años recientes la literatura sobre pronóstico ha avanzado en varias direcciones para lidiar con estas dificultades. Por un lado, autores como Clements y Hendry (2006) proponen una batería de modelos para pronóstico que permiten considerar quiebres en la media y cambios de tendencia determinística. También en los últimos años se ha extendido el uso de modelos que utilizan un número grande de predictores para pronósticos siguiendo dos caminos: (i) el pooling de pronósticos, que combina mediante distintos tipos de ponderaciones, un número considerable de modelos y (ii) los factor models, que permiten encontrar medidas resumidas de la variabilidad de un gran número de series relevantes del ciclo económico. En el primer caso el camino elegido va en la dirección de preservar los modelos causales y enventualmente lograr un mejor pronóstico ampliando el conjunto de predictores. En el segundo caso se considera un gran conjunto de series del ciclo económico y mediante la técnica de estadísticas multivariada de de componentes principales se extrae un número reducido de factores subyacentes a esas series que explican una porción significativa de su variabilidad conjunta. La evidencia empírica indica que estas variables adicionan información relevante.

\subsubsection{Pooling de pronósticos}

El pooling o combinación de pronósticos implica combinar dos o más pronósticos provenientes de modelos que usan distintos predictores para producir un pronóstico. Esta técnica fue originalmente desarrollada por Bates y Granger (1969) y la idea básica es la siguiente: ${ }^{2}$

Sea $\left\{Y_{i, t+h}^{h}, i=1, \ldots . ., n\right\}$ un panel de $n$ pronósticos. El pronóstico combinado o pool de pronósticos estará dado por la combinación lineal

$$
Y_{t+h / t}^{h}=w_{0}+\sum_{i=1}^{n} w_{i t} Y_{i, t+h / t}^{h}
$$

\footnotetext{
${ }^{2}$ Una descripción detallada de la técnica de pooling de pronóstico y los principales desarrollos de esta literatura puede encontrarse en Stock y Watson (2006) y aún en más detalle en Timmerman (2006).
} 
donde $w_{i t}$ es el peso del iésimo pronóstico en el período $t$.

Bates y Granger (1969) muestran que los pesos que minimizan el error cuadrático medio de pronóstico $(R M S E)$ están dados por la proyección a la población de $Y_{t+h / t}^{h}$ en una constante y los pronósticos individuales. Frecuentemente se omite la constante e imponiendo $\sum_{i=1}^{n} w_{i t}=1$ se tiene que si cada uno de los pronósticos es insesgado, $Y_{t+h / t}^{h}$ también lo es. En la medida que ninguno de los pronósticos es generado por el verdadero modelo, la combinación óptima de pronósticos reparte el peso en una combinación múltiple de pronósticos. El mínimo $R M S E$ combinando esos pronósticos será variable en el tiempo si las matrices de varianzas y covarianzas de $\left(Y_{t+h / t}^{h},\left\{Y_{i, t+h / t}^{h}\right\}\right)$ cambian en el tiempo.

En la práctica, los ponderadores óptimos no son viables, porque las matrices de varianzas y covarianzas son desconocidas. Granger y Ramanathan (1984) proponen estimar los ponderadores utilizando mínimos cuadrados ordinarios o mínimos cuadrados restringidos, si se impone $w_{0}=0$ y $\sum_{i=1}^{n} w_{i t}=1$, aunque si $n$ es grande se espera que las estimaciones tengan un desempeño pobre, simplemente por el hecho de que al estimarse un gran número de parámetros se está introduciendo incertidumbre muestral. Si $n$ es propocional al tamaño de la muestra, el estimador de MCO no es consistente y las combinaciones que lo utilizan no son asintóticamente óptimas. Por esa razón la investigación en la combinación o pooling de pronósticos se ha focalizado en imponer más estructura a la combinación de pronósticos. Entre las posibles técnicas utilizadas se tienen:

(i) Combinación simple de pronósticos: reporta una medida del centro de la distribución del panel de pronósticos. Los pesos están igualmente distribuídos, es decir, $w_{i t}=1 / n$. La combinación mediana o la media truncada son combinaciones simples menos sensibles a la presencia de observaciones extremas.

(ii) Pesos basados en el error cuadrático medio (RMSE): el pronóstico combinado utiliza ponderadores que dan peso a los pronósticos dependiendo inversamente de sus RMSE descontados

$$
w_{i t}=m_{i t}^{-1} / \sum_{j=1}^{n} m_{j t}^{-1}, \quad \text { donde } m_{i t}=\sum_{s=T_{0}}^{t-h} \rho^{t-h-s}\left(Y_{s+h}^{h}-\widehat{Y}_{s+h / s}^{h}\right)^{2}
$$

donde $\rho$ es un factor de descuento.

Utilizamos aqui una variante de los pesos basados en el $R M S E$ propuesta por Marcellino (2002).

(iii) Shrinkage de pronósticos: técnica que involucra una convergencia de los ponderadores hacia algún valor a priori que usualmente suele ser pesos iguales.

Diebold y Pauly (1990) proponen usar 


$$
w_{i t}=\lambda \widehat{w}_{i t}+(1-\lambda)(1 / n)
$$

donde $\widehat{w}_{i t}$ es el coeficiente estimado en una regresión de $M C O$ de $Y_{s+h}^{h}$ en $\quad \widehat{Y}_{1, s+h / s}^{h}$ , ......., $\widehat{Y}_{n, s+h / s}^{h}$ para $s=T_{0}, \ldots . . t-h$, donde $T_{0}$ es la primera fecha del pronóstico combinado y $\lambda$ controla por el grado de shrinkage hacia pesos iguales.

\subsubsection{Dynamic Factor Models}

El desarrollo de los Dynamic Factor models se basa en el análisis factorial y el análisis de componentes principales, técnicas de larga data en el análisis estadístico multivariado. La idea que subyace a estas técnicas es que la covariación entre un gran número $n$ de series económicas en sus rezagos y adelantos puede ser representada por un número reducido de factores $q$ no observados, con $n>q$. Las perturbaciones a esos factores podrían representar en este contexto, shocks sobre la demanda o la oferta agregada.

Se tiene entonces que el vector de $n$ series observadas del ciclo puede ser explicada por los rezagos distribuidos de $q$ factores comunes más las $n$ perturbaciones idiosincráticas que eventualmente podrían estar serialmente correlacionadas y también correlacionadas entre los $i$.

$$
X_{i t}=\lambda_{i}(L)^{\prime} f_{t}^{\prime}+u_{i t}
$$

Donde $f_{t}$ es un vector $q \times 1$ de factores no observados, $\lambda$ es un vector $q \times 1$ de polinomios de rezagos de dynamic factor loadings y los $u_{i t}$ son las perturbaciones idiosincrásicas, que se supone no están serialmente correlacionadas con los factores en todos los rezagos y adelantos, es decir $E\left(f_{t} u_{i t}\right)=0$ para todo $i, s$.

El objetivo es entonces estimar $E\left(y_{t+1} / X_{t}\right)$ modelando a $y_{t+1}$ según

$$
y_{t+1}=\beta_{t}^{\prime} F_{t}+\varepsilon_{t+1}
$$

donde $F_{t}=\left[f_{t}^{\prime}, f_{t-1 . \cdots}^{\prime} \ldots f_{t-p}^{\prime}\right]$ es un vector de dimensión $r=(p+1) \bar{r}$ donde $p$ es el número máximo de rezagos, y $\bar{r}$ el número predefinido de factores a ser extraídos de los datos. Si los polinomios de rezagos $\lambda_{i t}(L)$ en 4 y $\beta(L)$ en 5 son de orden finito $p$, Stock y Watson (2002a) muestran que los factores $F$ pueden ser estimados por el método de componentes principales y modelar a $y_{t+1}$ según la ecuación $6 .^{3}$

$$
Y_{t+1}=\beta^{\prime} F_{t}+\varepsilon_{t+1}
$$

\footnotetext{
${ }^{3}$ Una breve descripción de la técnica multivariada de componentes principales se presenta en el Apéndice A.
} 


\subsection{Los resultados empíricos}

Dados los grandes cambios que experimentó la tasa media de inflación en Argentina y la volatilidad asociada a este fenómeno, nos inclinamos por considerar para el pronóstico una muestra comprendida entre 1993.1 y 2006.12. Si bien este período incluye dos sub-períodos bastante diferentes tanto en términos del régimen monetario vigente como de la dinámica de la inflación (ver al respecto la sección 6 en el Capítulo II) consideramos que esos cambios no son de una magnitud que dificulte de manera importante la estimación y el pronóstico de inflación.

Evaluamos la capacidad predictiva de un conjunto de modelos de pronóstico que incluye: un modelo $A R M A$ y varios modelos multivariados: un $V A R$ monetario bivariado, una curva de Phillips híbrida y dos modelos que proyectan la inflación en base a factores obtenidos estos últimos utilizando el método de componentes principales. Evaluamos la capacidad predictiva de los modelos mediante pronósticos out of sample.

En general la estructura de rezagos fue elegida siguiendo los criterios convencionales de Akaike y Schwarz.

Para evaluar la capacidad de pronóstico de los modelos se calcularon el error cuadrático medio $(R M S E)$, el error medio absoluto $(M A E)$, el error medio absoluto porcentual $(M A P E)$ también el ratio del $R M S E$ de cada modelo respecto del elegido como benchmark que es el modelo $A R M A$.

Se encontró que un $A R M A(1,12)$ puede ser una representación adecuada para la inflación en el período 1993 -2006. Se utilizaron variables dummy para controlar por observaciones extremas.

Respecto de los modelos causales, se eligieron dos que incorporan variables cuya elección refleja teorías alternativas de la inflación. Por un lado curva de Phillips neokeynesiana híbrida, especificada para el caso de

${ }^{4}$ que adiciona a la inflación esperada un término de la inflación rezagada e incluye como determinantes de la inflación, la brecha del producto, la depreciación nominal y una medida de la inflación internacional,

Por otro lado se estima un modelo $V A R$ monetario que incluye la inflación y el cambio en el agregado monetario $M 2$, como una medida del dinero transaccional. En este caso el modelo refleja la noción de que el dinero debería ser un determinante de la tasa de inflación en el largo plazo.

Adicionalmente a los modelos mencionados se estimaron modelos en base a factores, siguiendo la metodología descripta en la sección 2.1. Los factores obtenidos a través

\footnotetext{
${ }^{4}$ Galí y Gertler (1999) proponen una curva de Phillips neo-keynesiana híbrida, que incorpora un término rezagado de la inflación. El modelo utilizado aqui para pronóstico fue estimado por D 'Amato y Garegnani (2006) y está en línea con el de Galí y Getler, aunque extendido al caso de una economía abierta pequeña. Ver también al respecto el Capítulo III de esta tesis.
} 
del método de componentes principales resumen la variabilidad conjunta de un gran número de series económicas que fueron agrupadas según se trata de series asociadas a la demanda agregada en factores de demanda, las asociadas a la oferta en factores de oferta y un conjunto de series nominales que incluye agregados monetarios, precios, tasas de interés y recaudación tributaria, entre otras cuya variabilidad se resumió en factores a los que llamamos nominales. Finalmente, todas las series fueron utilizadas en forma conjunta para construir factores a los que denominamos factores totales. Sólo los modelos estimados en base a los factores totales y nominales resultaron satisfactorios para explicar la dinámica de la inflación. ${ }^{5}$ En general no se utilizó más allá del cuarto componente principal, de acuerdo a lo sugerido por la literatura. El modelo estimado es del tipo

$$
Y_{t+1}=\beta^{\prime} F_{t}+\varepsilon_{t+1}
$$

donde $F_{t}=\left[f_{t}^{\prime}, f_{t-1 .}^{\prime} \ldots f_{t-p}\right]$ es un vector de dimensión $r=(p+1) \bar{r}$ donde $p$ es el número máximo de rezagos, y $\bar{r}$ el número de predefinido de factores a ser extraídos de los datos, que en nuestro caso es 4 .

Un detalle de los modelos estimados se presenta en el Apéndice C.

\subsubsection{La capacidad predictiva de los modelos estimados}

Para comparar el desempeño de los distintos modelos reportamos cuatro tipos de estadísticos: el error cuadrático medio $(R M S E)$, el error absoluto medio $(M A E)$, el error porcentual absoluto medio $(M A P E)$ y el estadístico $U-T h e i l$, que compara el $R M S E$ de cada modelo con el del mejor modelo univariado elegido como benchmark. Los resultados se muestran en la Tabla 1.

\footnotetext{
${ }^{5}$ Ver Apéndice B para una descripción de las series que se consideraron para obtener los distintos factores.
} 
Tabla 1: capacidad predictiva de modelos individuales

\begin{tabular}{|c|c|c|c|c|c|}
\hline \multicolumn{6}{|c|}{ RMSE } \\
\hline $\begin{array}{l}\text { Período de } \\
\text { pronóstico }\end{array}$ & $\operatorname{ARMA}(1,12)$ & $\begin{array}{c}\text { Curva de } \\
\text { Phillips-GMM }\end{array}$ & VAR & Factores totales & $\begin{array}{c}\text { Factores } \\
\text { nominales }\end{array}$ \\
\hline 2006:10-2006:12 & 0.0027 & 0.0036 & 0.0031 & 0.0034 & 0.0046 \\
\hline 2006:7-2006:12 & 0.0026 & 0.0039 & 0.0028 & 0.0034 & 0.0043 \\
\hline $2006: 1-2006: 12$ & 0.0033 & 0.0037 & 0.0032 & 0.0036 & 0.0039 \\
\hline \multicolumn{6}{|c|}{ MAE } \\
\hline $\begin{array}{l}\text { Período de } \\
\text { pronóstico }\end{array}$ & $\operatorname{ARMA}(1,12)$ & $\begin{array}{l}\text { Curva de } \\
\text { Phillips }\end{array}$ & VAR & Factores totales & $\begin{array}{c}\text { Factores } \\
\text { nominales }\end{array}$ \\
\hline 2006:10-2006:12 & 0.0024 & 0.0035 & 0.0031 & 0.0029 & 0.0043 \\
\hline $2006: 7-2006: 12$ & 0.0022 & 0.0033 & 0.0027 & 0.0028 & 0.0038 \\
\hline 2006:1-2006:12 & 0.0027 & 0.0032 & 0.0025 & 0.0027 & 0.0031 \\
\hline \multicolumn{6}{|c|}{ MAPE } \\
\hline $\begin{array}{l}\text { Período de } \\
\text { pronóstico }\end{array}$ & $\operatorname{ARMA}(1,12)$ & $\begin{array}{l}\text { Curva de } \\
\text { Phillips }\end{array}$ & VAR & Factores totales & $\begin{array}{c}\text { Factores } \\
\text { nominales }\end{array}$ \\
\hline 2006:10-2006:12 & 27.33 & 43.69 & 36.67 & 32.96 & 49.61 \\
\hline $2006: 7-2006: 12$ & 26.93 & 40.49 & 35.32 & 34.95 & 47.74 \\
\hline $2006: 1-2006: 12$ & 36.15 & 45.08 & 31.39 & 31.51 & 36.67 \\
\hline \multicolumn{6}{|c|}{ estadístico U-Theil } \\
\hline $\begin{array}{l}\text { Período de } \\
\text { pronóstico }\end{array}$ & $\operatorname{ARMA}(1,12)$ & $\begin{array}{l}\text { Curva de } \\
\text { Phillips }\end{array}$ & VAR & Factores totales & $\begin{array}{c}\text { Factores } \\
\text { nominales }\end{array}$ \\
\hline 2006:10-2006:12 & 1 & 1.33 & 1.15 & 1.23 & 1.67 \\
\hline 2006:7-2006:12 & 1 & 1.53 & 1.09 & 1.30 & 1.66 \\
\hline $2006: 1-2006: 12$ & 1 & 1.12 & 0.97 & 1.08 & 1.18 \\
\hline
\end{tabular}

Una pregunta inmediata es si el modelo univariado supera en forma sistemática a los modelos causales y los multivariados en su capacidad de pronóstico. La respuesta es algo mixta. En un horizonte muy corto, como un trimestre o un semestre, el modelo $A R M A$ supera en forma sistemática al resto de los modelos (el estadístico $U-T h e i l$ supera 1). Cuando se extiende el horizonte a un año el $V A R$ monetario supera al $A R M A$ (estadístico $U$ - Theil 0.97). En un horizonte de un año el $V A R$ monetario está muy cerca del mejor modelo univariado y supera al modelo $A R M A$.

En forma resumida, los resultados indican una complementariedad entre los modelos de pronóstico que puede ser explotada en la medida que se la identifique. Los modelos univariados tienen un muy buen desempeño en horizontes muy cortos. A medida que se extiende el horizonte de pronóstico, los modelos multivariados, tanto aquellos cuyo uso se sustenta en la teoría como los que utilizan factores como medidas resumen de la variablidad conjunta de distintos indicadores del ciclo, se acercan en su desempeño a los modelos univariados y eventualmente los superan.

\subsubsection{Comparando la capacidad predictiva de modelos alternativos}

La evaluación de la capacidad predictiva de los modelos de pronóstico es importante no sólo porque el pronóstico se utiliza en general para la toma de decisiones de política, 
sino también porque implica una elección entre hipótesis económicas alternativas.

Como enfatizan Diebold y Mariano (1995), una revisión de la literatura empírica sobre pronóstico permite observar que la evaluación de la capacidad de pronóstico de modelos alternativos se basa usualmente en la comparación de estimaciones puntuales, sin una evaluación de la incertidumbre muestral. El uso de tests estadísticos se dificulta debido a que usualmente existen problemas de correlación serial en los errores de pronóstico o contemporánea entre los errores de pronóstico de los distintos modelos.

Diebold y Mariano proponen un conjunto de tests para evaluar la hipótesis nula de ausencia de diferencias en la capacidad predictiva de dos modelos de pronóstico alternativos. Los tests se basan en la evaluación de la presencia de diferencias signficativas entre los modelos y los datos. Los tests propuestos por Diebold y Mariano permiten, en algunos casos, errores de pronóstico no normales, serialmente correlacionados y también correlacionados entre sí en forma contemporánea.

Evaluamos aquí los modelos descriptos en la subsección anterior utilizando algunos de los tests propuestos por Dieblod y Mariano. Debido a que el ejercicio de pronóstico que se desarrolla considera horizontes no superiores a un año, la evaluación para todos los horizontes se basa principalmente en tests no paramétricos. En el caso de los pronósticos a un año también se evalúa la hipótesis de no diferencia de capacidad predictiva con tests paramétricos.

En muchas aplicaciones en las que se compara la capacidad predictiva de diferentes modelos la función de pérdida de cada modelo suele representarse por el error de pronóstico o bien en forma general por una función directa del mismo. En este trabajo se considera al propio error de pronóstico como función de pérdida del respectivo modelo. La hipótesis nula de igual capacidad predictiva para dos pronósticos será la distancia entre ambos errores de pronóstico. Para los tres horizontes de pronóstico considerados se utilizan tests adecuados al caso de pocas observaciones de errores de pronóstico: Dos de estos tests están basados en los diferenciales de pérdida observados (el test del signo) o en sus rango (test de signo de rangos de Wilcoxon). Para el caso de un año se complementan los tests previos con tests paraméticos que provee la literatura: un simple test F y el test de Morgan-Granger-Newbold (MGN). En el caso de estos dos últimos tests la función de pérdida está definida como el cuadrado de la distancia entre ambos errores de pronóstico. ${ }^{6}$

Los tests no paramétricos, de signo y de signo de rango de Wilcoxon permiten trabajar con todos los horizontes. En la Tabla 2 se reporta el número de diferencias positivas observadas para los pronósticos de todos los modelos comparados con el modelo ARMA. Por ejemplo, en el caso de la curva de Phillips y para un horizonte de 1 año, el número 5 en la columna del test de signos indica que de las 12 diferencias del

\footnotetext{
${ }^{6}$ En el Apéndice D se presenta una descripción breve de los tests implementados.
} 
error de pronóstico de la curva de Phillips respecto del $A R M A, 5$ son positivas. De acuerdo a una distribución binomial con parámetros $T=12$ y $1 / 2$, bajo la hipótesis nula, ambos modelos no se diferencian en su capacidad predictiva. Este resultado se repite para el resto de los modelos y horizontes de pronóstico. En el caso del test de signo de rangos de Wilcoxon la versión estudentizada permite utilizar la distribución normal estándar y al $5 \%$ de significatividad no se rechaza la hipótesis de igual capacidad predictiva, con excepción del modelo de factores nominales para el horizonte de 1 año. El rechazo implica en este caso que el modelo ARMA supera en capacidad predictiva al de factores nominales. ${ }^{7}$

Los tests paramétricos requieren del cumplimiento de algunos supuestos respecto de los errores de pronóstico: (i) que la función de pérdida sea cuadrática; (ii) que los errores de pronóstico tengan media 0 (iii) que tengan una distribución normal; (iv) no estén correlacionados serialmente y (v) no se correlacionen contemporánemente entre sí. El test MGN permite correlación contemporánea entre los errores de pronóstico. En el caso del test F, cuando se comparan los valores del estadístico observado con los valores críticos para una $\mathrm{F}(12,12)$, se encuentra que no se rechaza la hipótesis nula al $5 \%$. Los resultados del test MGN no son muy diferentes cuando se evalúa la hipótesis nula considerando la distribución t de student con T-1 grados de libertad. Es decir, para ambos tests la capacidad predictiva de los modelos no es estadísticamente distinta respecto del modelo ARMA.

Tabla 2

\begin{tabular}{|c|c|c|c|c|}
\hline $\begin{array}{l}\text { Compar } \\
\text { Modelo de pronóstico }\end{array}$ & $\begin{array}{l}\text { ción de ca } \\
\text { Test de } \\
\text { Signo (1) }\end{array}$ & $\begin{array}{l}\text { Dacidad predictiva respe } \\
\text { Test de signo de rangos } \\
\text { de Wilcoxon (2) }\end{array}$ & $\begin{array}{l}\text { le Mode } \\
\text { Test F }\end{array}$ & $\begin{array}{l}\text { MA } \\
\text { Test de Morgan- } \\
\text { Granger-Newbold }\end{array}$ \\
\hline \multicolumn{5}{|l|}{ Curva de Phillips } \\
\hline 3 meses & 2 & & & \\
\hline 6 meses & 4 & -1.3628 & & \\
\hline 1 año & 5 & -0.3922 & 1.3845 & 1.0635 \\
\hline \multicolumn{5}{|l|}{ VAR monetario } \\
\hline 3 meses & 2 & & & \\
\hline 6 meses & 3 & -1.5724 & & \\
\hline 1 año & 5 & -0.4707 & 0.9362 & 0.1390 \\
\hline \multicolumn{5}{|l|}{ Factores totales } \\
\hline 3 meses & 2 & & & \\
\hline 6 meses & 4 & -1.3628 & & \\
\hline 1 año & 7 & 0.4707 & 1.4020 & 0.4186 \\
\hline \multicolumn{5}{|l|}{ Factores nominales } \\
\hline 3 meses & 3 & & & \\
\hline 6 meses & 6 & -0.9435 & & \\
\hline 1 año & 10 & 2.3534 & 1.7828 & 0.4844 \\
\hline
\end{tabular}

(1) Parámetros de la distribución binomial T (cantidad de períodos a pronosticar) y 1/2

(2) Estadístico basado en Wilcoxon es su versión asintótica normal estudentizada

\footnotetext{
${ }^{7} \mathrm{El}$ test se calculó para los horizontes de 6 y 12 meses, dado que requiere simetría en la función de pérdida.
} 
En resumen, los tests indican que ningún modelo supera para todos los horizontes al resto en capacidad predictiva. Este resultado sugeriría la posibilidad de trabajar con una combinación o pooling de pronósticos, teniendo en cuenta que cada uno de los modelos considerados puede contener información relevante para el pronóstico. También se puede pensar que estos modelos pueden ser complementarios en el pronóstico en el sentido que permiten responder a distintas preguntas y a guiar diferentes decisiones de política. Por ejemplo, el $V A R$ monetario permite responder a un pregunta relevante como cuál es el rezago con que los impulsos monetarios se transmiten a los precios o la curva de Phillips es informativa acerca del impacto de cambios en la brecha del producto sobre la tasa de inflación. Timmermann (2003) señala que, a menos que uno pueda identificar ex-ante un modelo con mejor capacidad predictiva que sus competidores, la combinación de pronósticos ofrece ganancias de diversificación que la hacen atractiva por sobre los pronósticos derivados de un modelo individual.

Considerar una combinación de estos pronósticos presenta ventajas en varios sentidos: (i) la combinación de pronósticos ofrece diversificación. Intuitivamente, si se tiene una función de pérdida cuadrática, aún cuando uno de los modelos supere al otro en capacidad predictiva, una combinación lineal de ellos, al generar una pérdida menor puede ser preferida; (ii) para economías sujetas a cambios estructurales la combinación de pronósticos provee una mejor predicción por sobre el mejor modelo individual. En general, las velocidades de adaptación a cambios estructurales de los modelos suele diferir. En ese sentido combinar modelos con distinta adaptabilidad al cambio puede superar a los modelos individuales (iii) La combinación de pronóstico puede ser vista como un modo de hacer más robusto el pronóstico frente al sesgo de especificación y los errores de medición de las variables de los pronósticos individuales. Por ejemplo, si dos pronósticos tiene sesgos diferentes, en direcciones opuestas, es fácil pensar que la combinación puede generar un mejora en el pronóstico. ${ }^{8}$

En base a lo sugerido por Marcellino (2002), construímos combinaciones lineales de pronósticos en base a promedios ponderados, con ponderadores calculados del siguiente modo

$$
\widehat{y}_{t+h}=\sum_{m=1}^{M} k_{m, h, t} \widehat{y}_{t+h, m}, \text { con } k_{m, h, t}=\left(\frac{1}{R M S E_{m, h, t}}\right)^{w} / \sum_{j=1}^{M}\left(\frac{1}{R M S E_{j, h, t}}\right)^{w}
$$

donde $m$ indexa los modelos, $k_{m, h, t}$ denota los ponderadores y $R M S E$ es el error cuadrático medio.

\footnotetext{
${ }^{8}$ Para una revisión detallada de las ventajas de la combianción de pronósticos ver Hendry y Clements (2004), Marcellino (2002) y Timmermann (2006).
} 
Los ponderadores de cada modelo son elegidos de manera inversamente proporcional a sus estadísticos de capacidad predictiva en el caso de w=1 en la ecuación 4 . También consideramos el caso de $w=5$, en el que una mayor ponderación se asigna a los modelos con mejor capacidad predictiva.

En nuestro caso las combinaciones se realizaron para todos los modelos y usando para las ponderaciones los tres estadísticos de capacidad predictiva antes mencionados: RMSE, MAE y $M A P E$.

En la Tabla 3 se presentan: los RMSE, los MAE, los MAPE y el $U$ - Theil para combinaciones ponderadas con $w=1$ y $w=5$ usando como ponderadores los respectivos $R M S E, M A E$ y $M A P E$ de cada modelo. El estadístico $U-T h e i l$ se computa relativo al $A R M A$.

Tabla 3

\begin{tabular}{|c|c|c|c|c|c|c|}
\hline \multirow[b]{2}{*}{ Período de pronóstico } & \multicolumn{3}{|c|}{$w=1$} & \multicolumn{3}{|c|}{$w=5$} \\
\hline & RMSE & MAE & MAPE & RMSE & MAE & MAPE \\
\hline 2006:10-2006:12 & 0.0032 & 0.0032 & 0.0031 & 0.0028 & 0.0028 & 0.0028 \\
\hline $2006: 7-2006: 12$ & 0.0030 & 0.0030 & 0.0030 & 0.0027 & 0.0027 & 0.0027 \\
\hline $2006: 1-2006: 12$ & 0.0034 & 0.0034 & 0.0034 & 0.0032 & 0.0033 & 0.0034 \\
\hline \multicolumn{7}{|c|}{ MAE } \\
\hline & \multicolumn{3}{|c|}{$w=1$} & \multicolumn{3}{|c|}{$w=5$} \\
\hline Período de pronóstico & RMSE & MAE & MAPE & RMSE & MAE & MAPE \\
\hline 2006:10-2006:12 & 0.0030 & 0.0030 & 0.0030 & 0.0024 & 0.0024 & 0.0023 \\
\hline 2006:7-2006:12 & 0.0027 & 0.0027 & 0.0027 & 0.0023 & 0.0023 & 0.0023 \\
\hline $2006: 1-2006: 12$ & 0.0027 & 0.0027 & 0.0027 & 0.0026 & 0.0026 & 0.0026 \\
\hline \multicolumn{7}{|c|}{ MAPE } \\
\hline & \multicolumn{3}{|c|}{$w=1$} & \multicolumn{3}{|c|}{$w=5$} \\
\hline Período de pronóstico & RMSE & MAE & MAPE & RMSE & MAE & MAPE \\
\hline 2006:10-2006:12 & 34.61 & 34.47 & 34.16 & 27.15 & 26.20 & 24.99 \\
\hline 2006:7-2006:12 & 55.79 & 55.69 & 55.81 & 46.37 & 45.40 & 45.50 \\
\hline $2006: 1-2006: 12$ & 32.53 & 32.45 & 32.44 & 31.28 & 30.92 & 30.95 \\
\hline \multicolumn{7}{|c|}{ estadístico U-Theil } \\
\hline & \multicolumn{3}{|c|}{$w=1$} & \multicolumn{3}{|c|}{$w=6$} \\
\hline Período de pronóstico & RMSE & MAE & MAPE & RMSE & MAE & MAPE \\
\hline 2006:10-2006:12 & 1.160 & 1.156 & 1.154 & 1.031 & 1.025 & 1.024 \\
\hline $2006: 7-2006: 12$ & 1.180 & 1.179 & 1.181 & 1.051 & 1.047 & 1.050 \\
\hline $2006: 1-2006: 12$ & 1.010 & 1.012 & 1.019 & 0.973 & 0.984 & 1.020 \\
\hline
\end{tabular}

Los resultados indican que la combinación de pronósticos supera al mejor pronóstico individual para un horizonte de un año y con ponderadores más que inversamente proporcionales de los mejores modelos individuales. Este resultado es de utilidad a los fines prácticos pues el horizonte de un año es el relevante para la toma de decisiones de política económica. La posibilidad de combinar modelos tanto univariados como multivariados permite además responder a preguntas específicas de política económica.

\subsection{Conclusiones}

Estimamos una variedad de modelos de inflación a los fines de pronóstico que incluyen desde modelos univariados, modelos causales, basados en teorías alternativas 
de la inflación a modelos basados en el uso de factores como medidas resumen de la variabilidad conjunta de un gran número de series económicas. Encontramos que si bien el modelo univariado es en general el de mejor desempeño, a medida que se extiende el horizonte de pronóstico, los modelos multivariados se acercan al desempeño de los univariados. En particular un $V A R$ monetario llega a superar al modelo ARMA univariado para el horizonte de un año, si se comparan las estimaciones puntuales de distintos estadísticos calculados para evaluar la capacidad predictiva de los modelos. Sin embargo, cuando se calculan tests para evaluar la significatividad estadística de las diferencias en capacidad predictiva de los modelos, tomando como benchmark un modelo $A R M A$ univariado, las diferencias no resultan estadísticamente significativas. Finalmente se combinan los modelos estimados mediante un pool de pronósticos utilizando como ponderadores los inversos de los RMSE de los respectivos modelos. Los resultados indican que alguna de las combinaciones de pronósticos supera al mejor pronóstico individual para un horizonte de un año. Teniendo en cuenta que el horizonte de una año es el relevante para la toma de decisiones de política económica, la posibilidad de combinar modelos tanto univariados como multivariados para pronóstico es interesante porque permite además responder a preguntas específicas de política económica. 


\subsection{Apéndice A: El método de componentes principales}

La metodología de componentes principales permite obtener una medida sintética de la variabilidad conjunta de una colección de variables aleatorias relacionadas, a partir de calcular aquellas combinaciones lineales de estas variables cuya varianza es máxima. ${ }^{9}$

Dado un conjunto de $n$ variables aleatorias que se conoce están relacionadas, se espera que un número relativamente reducido de combinaciones lineales de éstas los componentes principales- pueda explicar una gran proporción de su variabilidad total. En ese sentido, el método permite resumir en un conjunto reducido de $q<n$ componentes principales y sus varianzas la información contenida en un vector $X: t x n$ de variables aleatorias y su matriz de varianzas y covarianzas $\Sigma$

Dado un vector de variables aleatorias $X: \operatorname{txn} \operatorname{con} E(X)=\Phi, \operatorname{var}(X)=\Sigma$ es posible definir $\alpha \equiv\left(\alpha_{i}\right)$ como un vector $n x 1$ de ponderadores desconocidos de los componentes de $X$ y $z$ como un vector tal que

$$
\begin{aligned}
z & =X^{\prime} \alpha \\
\operatorname{con} z_{i} & =\sum_{i=1}^{n} \alpha_{i} X_{i}
\end{aligned}
$$

Si los elementos de $X$ están medidos en las mismas unidades es posible imponer:

$$
\alpha^{\prime} \alpha=\sum_{i=1}^{n} \alpha_{i}=1
$$

De esta manera es posible encontrar un vector de ponderadores $\alpha$ tal que maximice $\operatorname{var}(z)=\alpha^{\prime} \Sigma \alpha$, sujeto a $\alpha^{\prime} \alpha=1$

Entonces el problema de:

$$
\begin{aligned}
& \operatorname{máx}_{\alpha}\left(\alpha^{\prime} \Sigma \alpha\right) \\
& \text { s.a. } \alpha^{\prime} \alpha=1
\end{aligned}
$$

puede escribirse como

$$
L=\alpha^{\prime} \Sigma \alpha-\lambda\left(\alpha^{\prime} \alpha-1\right)
$$

Diferenciando $L$ con respecto a $\alpha$ e igualando a 0 se tiene que

\footnotetext{
${ }^{9}$ Para una descripción detallada de la técnica de análisis multivariado de componentes principales ver Press (1972) y Kendall (1975).
} 


$$
\frac{\partial L}{\partial \alpha}=2 \Sigma \alpha-2 \lambda \alpha=0
$$

y

$$
(\Sigma-\lambda I) \alpha=0
$$

y dado que

$$
\alpha \neq 0
$$

Existe una solución si

$$
|\Sigma-\lambda I|=0
$$

Esto implica que $\lambda$ es una raíz característica de la matriz de varianzas y covarianzas de $X, \Sigma$, y $\alpha$ es un vector característico de esa matriz. Dado que $\Sigma$ es una matriz $n \times n$ existen $n$ raíces características $\lambda$ que satisfacen 3 y $n$ combinaciones lineales ortogonales asociadas a $n$ vectores característicos $\alpha$. Teniendo en cuenta 2

$$
\Sigma \alpha=\lambda \alpha
$$

y premultiplicando por $\alpha^{\prime}$

$$
\alpha^{\prime} \Sigma \alpha=\lambda \alpha^{\prime} \alpha=\lambda
$$

El mayor valor de $\lambda$ es aquel que maximiza la varianza de $z$. La solución al problema 1 está dada por $\left(\alpha_{1}, \lambda_{1}\right)$; y $z_{1}$ se conoce como el primer componente principal de ese conjunto de variables aleatorias. 


\subsection{Apéndice B: Detalle de las series del ciclo utilizadas} para el cálculo de factores

Todas las series utilizadas han sido desestacionalizadas utilizando el programa X-12 ARIMA y posteriormente estandarizadas.

Factor de demanda

\begin{tabular}{|l|c|}
\hline \multicolumn{1}{|c|}{ Series } & Factor \\
\hline ISE (indicador sintético de energía) desestacionalizado & Demanda/consumo \\
\hline SupermercadosVolumen de ventas a precios constantes (desestacionalizada) & Demanda/consumo \\
\hline Centros de Compras Volumen de ventas a precios constantes (desestacionali & Demanda/consumo \\
\hline Estadísticas de Servicios Públicos - Índice Sintético General & Demanda/consumo \\
\hline Importaciones desestacionalizadas & Demanda/consumo \\
\hline Automotores al Merc. Interno ventas unidades & Demanda/consumo \\
\hline Automotores Prod. Nac. al Merc. Interno ventas unidades & Demanda/consumo \\
\hline Automóviles Importaciones unidades & Demanda/consumo \\
\hline Ventas Azúcar Miles tns. & Demanda/consumo \\
\hline Ventas Cerveza Miles hls. & Demanda/consumo \\
\hline Ventas Vino Miles hls. & Demanda/consumo \\
\hline Ventas Gaseosas Miles hls. & Demanda/consumo \\
\hline Ventas Cigarrillos Mill. paquet. & Demanda/consumo \\
\hline Ventas Productos Farmacéut. Mill. unidad. & Demanda/consumo \\
\hline Ventas Naftas Miles m3 & Demanda/consumo \\
\hline Ventas Cemento Desp.al merc.int. Miles tns. & Demanda/consumo \\
\hline Ventas Asfalto Miles TN & Demanda/consumo \\
\hline Ventas Automóviles Unidades & Demanda/consumo \\
\hline Ventas Automóviles Utilitarios Unidades & Demanda/consumo \\
\hline Ventas Automóviles Carga y Pasaj. Unidades & Demanda/consumo \\
\hline Ventas Energía Demandada GWh & Demanda/consumo \\
\hline & \\
\hline
\end{tabular}


Factor de oferta

\begin{tabular}{|l|c|}
\hline UCI FIEL industria manufacturera & Produción/Oferta \\
\hline UCI FIEL bines de consumo no durables & Produción/Oferta \\
\hline UCI FIEL bines de consumo durables & Produción/Oferta \\
\hline UCI FIEL bienes de capital & Produción/Oferta \\
\hline UCI FIEL bienes de uso intermedio & Produción/Oferta \\
\hline Encuesta FIEL situacion general industria manufacturera & Produción/Oferta \\
\hline Encuesta FIEL situacion general bienes de consumo no durables & Produción/Oferta \\
\hline Encuesta FIEL situacion general bienes de consumo durables & Produción/Oferta \\
\hline Encuesta FIEL situacion general bienes de capital & Produción/Oferta \\
\hline Encuesta FIEL situacion general bienes de uso intermedio & Produción/Oferta \\
\hline Encuesta FIEL perspectiva proximos meses industria manufacturera & Produción/Oferta \\
\hline Encuesta FIEL perspectiva proximos meses bienes de consumo no durables & Produción/Oferta \\
\hline Encuesta FIEL perspectiva proximos meses bienes de consumo durables & Produción/Oferta \\
\hline Encuesta FIEL perspectiva proximos meses bienes de capital & Produción/Oferta \\
\hline Encuesta FIEL perspectiva proximos meses bienes de uso intermedio & Produción/Oferta \\
\hline Encuesta FIEL tendencia de demanda industria manufacturera & Produción/Oferta \\
\hline Encuesta FIEL tendencia de demanda bienes de consumo no durables & Produción/Oferta \\
\hline Encuesta FIEL tendencia de demanda bienes de consumo durables & Produción/Oferta \\
\hline Encuesta FIEL tendencia de demanda bienes de capital & Produción/Oferta \\
\hline Encuesta FIEL tendencia de demanda bienes de uso intermedio & Produción/Oferta \\
\hline Encuesta FIEL nivel de stocks industria manufacturera & Produción/Oferta \\
\hline Encuesta FIEL nivel de stocks bienes de coEncuesta FIEL nivel de stocksun & Produción/Oferta \\
\hline Encuesta FIEL nivel de stocks bienes de coEncuesta FIEL nivel de stocksun & Produción/Oferta \\
\hline Encuesta FIEL nivel de stocks bienes de capital & Produción/Oferta \\
\hline Encuesta FIEL nivel de stocks bienes de uso intermedio & Produción/Oferta \\
\hline
\end{tabular}

Factor de oferta (continuación)

\begin{tabular}{|l|l|}
\hline Automóviles Exportaciones unidades & Produción/Oferta \\
\hline EMI (estimador mensual de industria) desestacionalizado & Produción/Oferta \\
\hline EMAE (estimador mensual de actividad económica) desestacionalizado & Produción/Oferta \\
\hline IPI (índice de producción industrial) nivel general & Produción/Oferta \\
\hline IPI (índice de producción industrial) bienes de consumo no durables & Produción/Oferta \\
\hline IPI (índice de producción industrial) bienes de consumo durables & Produción/Oferta \\
\hline IPI (índice de producción industrial) bines de uso intermedio & Produción/Oferta \\
\hline IPI (índice de producción industrial) bienes de capital & Produción/Oferta \\
\hline IPI (índice de producción industrial) alimentos y bebidas & Produción/Oferta \\
\hline IPI (índice de producción industrial) cigarrillos & Produción/Oferta \\
\hline IPI (índice de producción industrial) insumos textiles & Produción/Oferta \\
\hline IPI (índice de producción industrial) pasta y papel & Produción/Oferta \\
\hline IPI (índice de producción industrial) combustible & Produción/Oferta \\
\hline IPI (índice de producción industrial) quimicos y plasticos & Produción/Oferta \\
\hline IPI (índice de producción industrial) minerales no metalicos & Produción/Oferta \\
\hline IPI (índice de producción industrial) siderurgia & Produción/Oferta \\
\hline IPI (índice de producción industrial) metalmecanica & Produción/Oferta \\
\hline IPI (índice de producción industrial) automóviles & Produción/Oferta \\
\hline Total Automotores producción unidades & Produción/Oferta \\
\hline Automóviles producción unidades & Produción/Oferta \\
\hline Cemento Despachos Totales & Produción/Oferta \\
\hline
\end{tabular}


Factor de oferta (continuación)

\begin{tabular}{|c|c|}
\hline Producción Hierro Primario Miles Tn. & Produción/Oferta \\
\hline Producción Acero Crudo Miles Tn. & Produción/Oferta \\
\hline Producción Petróleo Crudo Miles M3 & Produción/Oferta \\
\hline Producción Petróleo Procesado Miles M3 & Produción/Oferta \\
\hline Producción Gas Natural Mill. M3 & Produción/Oferta \\
\hline Producción Harina de Trigo Miles Tns. & Produción/Oferta \\
\hline Producción Aceites Vegetales Miles Tns. & Produción/Oferta \\
\hline Producción Subproductos Oleaginosos Miles Tns. & Produción/Oferta \\
\hline Producción Galletitas y Bizcochos Miles Tns. & Produción/Oferta \\
\hline Producción Faena Carne Vacuna Miles cab. & Produción/Oferta \\
\hline Producción Faena Aves Mills. aves & Produción/Oferta \\
\hline Producción Bebidas Espirituosas Miles Litros & Produción/Oferta \\
\hline Producción Hilados Celulósicos Tns. & Produción/Oferta \\
\hline Producción Pastas Para Papel Miles Tns. & Produción/Oferta \\
\hline \begin{tabular}{|l} 
Producción Papel para Diarios Toneladas \\
\end{tabular} & Produción/Oferta \\
\hline Producción Jabones Tocad y Lavar Toneladas & Produción/Oferta \\
\hline Producción Neumáticos Miles Unid. & Produción/Oferta \\
\hline Producción Urea Miles Tns. & Produción/Oferta \\
\hline Producción Soda Caústica Miles Tns. & Produción/Oferta \\
\hline Producción P.V.C. Miles Tns. & Produción/Oferta \\
\hline Producción Etileno Miles Tns. & Produción/Oferta \\
\hline Producción Polietileno Miles Tns. & Produción/Oferta \\
\hline Producción Polipropil. Miles Tns. & Produción/Oferta \\
\hline Producción Sec-Butanol Ton. & Produción/Oferta \\
\hline Producción Isopropanol Ton. & Produción/Oferta \\
\hline Producción Acido Sulfúrico Miles Tns. & Produción/Oferta \\
\hline Producción Cloro Miles Tns. & Produción/Oferta \\
\hline Producción Naftas Miles M3 & Produción/Oferta \\
\hline Producción Gas Oil Miles M3 & Produción/Oferta \\
\hline Producción Fuel Oil Miles Tns. & Produción/Oferta \\
\hline Producción Caucho Sint. Ton. & Produción/Oferta \\
\hline Producción Negro de Humo Ton. & Produción/Oferta \\
\hline Producción Pinturas para Construcción Ton. & Produción/Oferta \\
\hline Producción Cemento Portland Miles Ton. & Produción/Oferta \\
\hline Producción Hierro redondo p/hormigón Ton. & Produción/Oferta \\
\hline Producción Automotores Utilitarios Unidades & Produción/Oferta \\
\hline Producción Automotores Carga y Pasaj. Unidades & Produción/Oferta \\
\hline Producción Lam. Frío Miles Tn. & Produción/Oferta \\
\hline Producción Laminados en Caliente No Planos Miles Tn. & Produción/Oferta \\
\hline Producción Laminados en Caliente Planos Miles Tn. & Produción/Oferta \\
\hline Producción Zink Electrolítico Ton. & Produción/Oferta \\
\hline Producción Tractores Unidades & Produción/Oferta \\
\hline
\end{tabular}


Factor nominal

\begin{tabular}{|c|c|}
\hline Tasas Depositos en pesos En Caja & Tasas/Precios/Nom \\
\hline Tasas A Plazo Fijo 30 a $59 \mathrm{~d}$. & Tasas/Precios/Nom \\
\hline Tasas 60 d. o más & Tasas/Precios/Nom \\
\hline Tasas Depositos en dolares En Caja & Tasas/Precios/Nom \\
\hline Tasas A Plazo Fijo 30 a 59 d. & Tasas/Precios/Nom \\
\hline Tasas 60 d. o más & Tasas/Precios/Nom \\
\hline ITCRM dic2001=100 & Tasas/Precios/Nom \\
\hline IPC Nivel general & Tasas/Precios/Nom \\
\hline IPC alimentos y bebidas & Tasas/Precios/Nom \\
\hline IPC indumentaria & Tasas/Precios/Nom \\
\hline IPC vivienda y serv. básicos & Tasas/Precios/Nom \\
\hline IPC equipamiento y mantenimiento del hogar & Tasas/Precios/Nom \\
\hline IPC atención médica y gastos para la salud & Tasas/Precios/Nom \\
\hline IPC transporte y comunicaciones & Tasas/Precios/Nom \\
\hline IPC esparcimiento & Tasas/Precios/Nom \\
\hline IPC educación & Tasas/Precios/Nom \\
\hline IPC otros bienes y servicios varios & Tasas/Precios/Nom \\
\hline Indice de precios internos al por mayor (IPIM) Nivel general & Tasas/Precios/Nom \\
\hline IPIM Productos nacionales Total & Tasas/Precios/Nom \\
\hline IPIM Productos nacionales Primarios & Tasas/Precios/Nom \\
\hline IPIM Productos nacionales Manufacturados y Energía eléctrica & Tasas/Precios/Nom \\
\hline IPIM Productos Importados & Tasas/Precios/Nom \\
\hline Indice de precios internos básicos al por mayor (IPIB) Nivel general & Tasas/Precios/Nom \\
\hline IPIB Productos nacionales Total & Tasas/Precios/Nom \\
\hline IPIB Productos nacionales Primarios & Tasas/Precios/Nom \\
\hline IPIB Productos nacionales Manufacturados y Energía eléctrica & Tasas/Precios/Nom \\
\hline IPIB Productos Importados & Tasas/Precios/Nom \\
\hline Indice de precios básicos del productor (IPP) Nivel general & Tasas/Precios/Nom \\
\hline IPP Productos nacionales Primarios & Tasas/Precios/Nom \\
\hline IPP Productos nacionales Manufacturados y Energía eléctrica & Tasas/Precios/Nom \\
\hline Recaudación Tributaria IVA dgi & Tasas/Precios/Nom \\
\hline Recaudación Tributaria derechos importación & Tasas/Precios/Nom \\
\hline ByM privado fin de mes & Tasas/Precios/Nom \\
\hline M1 amplio privado fin de mes & Tasas/Precios/Nom \\
\hline M2 amplio privado fin de mes & Tasas/Precios/Nom \\
\hline M3 amplio privado fin de mes & Tasas/Precios/Nom \\
\hline Merval al cierre del mes & Tasas/Precios/Nom \\
\hline Merval promedio mensual & Tasas/Precios/Nom \\
\hline ByM total promedio de mes & Tasas/Precios/Nom \\
\hline M1 amplio total promedio de mes & Tasas/Precios/Nom \\
\hline M2 amplio total promedio de mes & Tasas/Precios/Nom \\
\hline M3 amplio total promedio de mes & Tasas/Precios/Nom \\
\hline Recaudación Tributaria IVA dga & Tasas/Precios/Nom \\
\hline
\end{tabular}




\subsection{Apéndice C: Modelos de pronóstico}

En la Tabla $C$ se presentan los modelos de pronóstico estimados

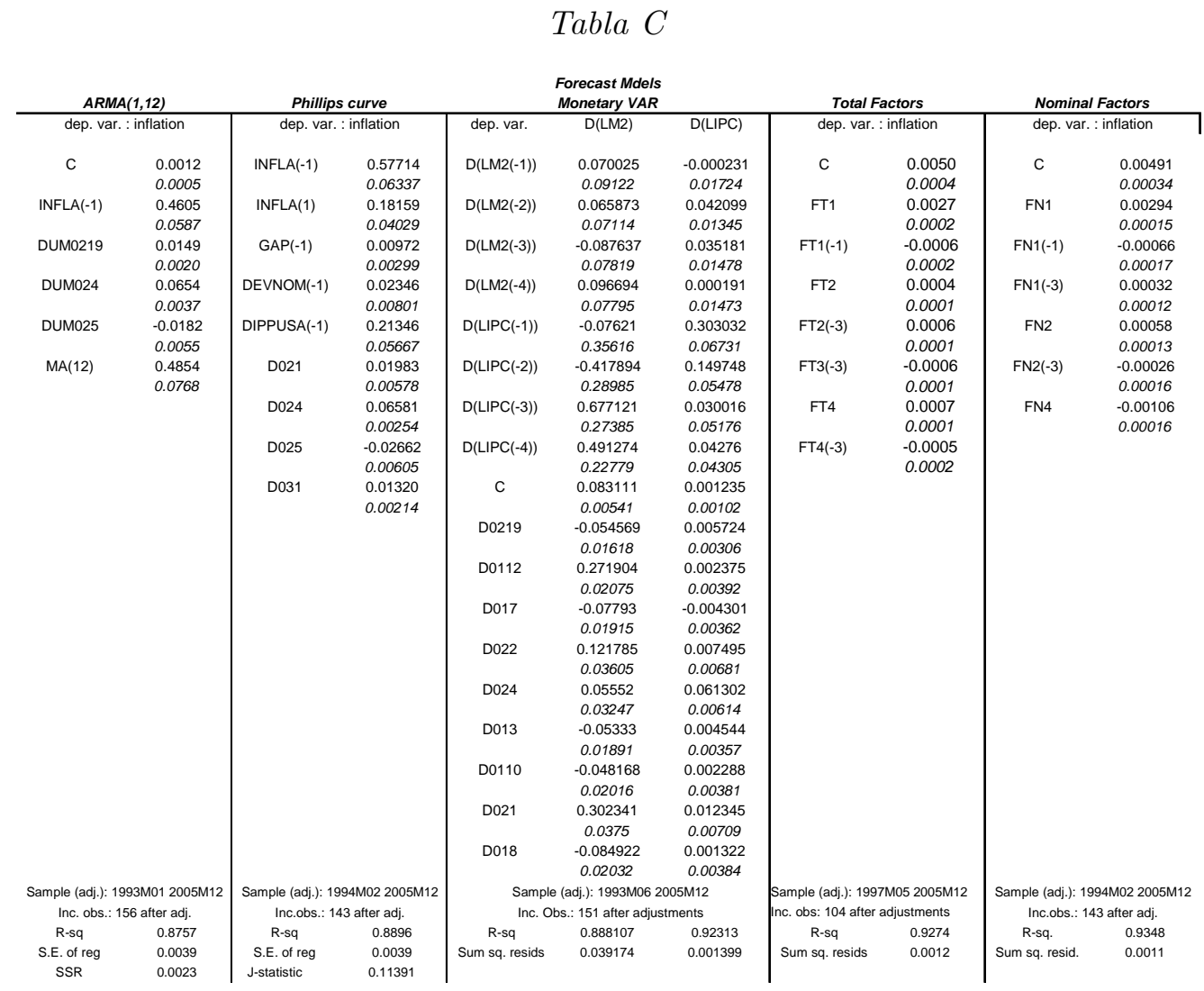




\subsection{Apéndice D: Tests para comparar capacidad predic- tiva}

\section{Test de Signo}

Dado el diferencial de pérdidas $d_{t}$, entre dos modelos $i$ y $j$, definido como

$$
d_{t}=\left[g\left(e_{i t}\right)-g\left(e_{j t}\right)\right]
$$

La hipótesis nula del test es que la mediana del diferencial de pérdidas es 0

$$
\operatorname{med}\left(g\left(e_{i t}\right)-g\left(e_{j t}\right)\right)=0
$$

Suponiendo que el diferencial de pérdidas es una variable $i i d$, el número de diferenciales positivos en una muestra de tamaño $T$ sigue una distribución binomial con parámetros $T, \frac{1}{2}$ bajo la hipótesis nula. El estadístico del test es entonces

$$
S_{1}=\sum_{t=1}^{T} I_{+}\left(d_{t}\right)
$$

donde

$$
\begin{aligned}
& I_{+}\left(d_{t}\right)=1 \quad \text { si } d_{t}>0 \\
& =0 \text { de lo contrario }
\end{aligned}
$$

La significatividad del estadístico puede verificarse en la tabla de la distribución binomial acumulada.

\section{Test de signo de rangos de Wilcoxon}

Este test no paramétrico requiere de simetría en el diferencial de pérdidas. Nuevamente se supone que el diferencial de pérdidas es $i i d$.

El estadístico del test es la suma de los rangos del valor absoluto de las diferencias positivas.

$$
S_{2}=\sum_{t=1}^{T} I_{+}\left(d_{t}\right) \operatorname{rango}\left(\left|d_{t}\right|\right)
$$

La distribución estudentizada del estadístico $S_{2}$ se distribuye asintóticamente como una normal estándar. 


$$
S_{2 s t d}=\frac{S_{2}-\frac{T(T+1)}{4}}{\sqrt{\frac{T(T+1)(2 T+1)}{24}}} \stackrel{a}{\sim} N(0,1)
$$

\section{Test $\mathbf{F}$}

Si (i) la pérdida es cuadrática y los errores de pronóstico (ii) tiene media 0 , (iii) siguen una distribución normal; (iv) no estén correlacionados serialmente y (v) no se correlacionen contemporánemente entre sí, el ratio de las varianzas muestrales sigue la distribución $\mathrm{F}$ bajo la hipótesis nula de no diferencia de capacidad predictiva. El estádístico a evaluar

$$
F=\frac{\frac{e^{\prime} e_{i}}{T}}{\frac{e_{j}^{\prime} e_{j}}{T}}=\frac{e_{i}^{\prime} e_{i}}{e_{j}^{\prime} e_{j}}
$$

se distribuye como una $F(T, T)$.

\section{Test de Morgan-Granger-Newbold}

Granger y Newbold (1977) buscan solucionar el problema de la correlación contemporánea entre los errores de pronóstico en base a una transformación ortogonal propuesta por Morgan (1939). Definiendo $x_{t}=\left(e_{i t}+e_{j t}\right)$ y $z_{t}=\left(e_{i t}-e_{j t}\right)$. Manteniendo los supuestos (i) a (iv), la nula de igual capacidad predictiva entre los modelos $i$ y $j$ es equivalente a una correlación 0 entre $x$ y $z$, es decir $\rho_{z x}=0$. El estadístico

$$
M N G=\frac{\widehat{\rho_{z x}}}{\sqrt{\frac{1-\widehat{\rho_{z x}}}{T-1}}}
$$

se distribuye como una t de Student con T-1 grados de libertad. 


\section{Bibliografía}

[1] Ahumada, H. y L. Garegnani, (2007), "Forecasting a monetary aggregate in a changing environment: Argentina after 2002", mimeo.

[2] Atkenson, A. y L. Ohanian, (2001) "Are Phillips Curve Useful for Forecasting Inflation". Federal Reserve Bank of Minneapolis, Quarterly Review, 25 (1), 2-11.

[3] Canova, F. (2002), "G-7 Inflation Forecasts", European Central Bank, WP N 151.

[4] Bates, J.M. y C.W.I. Granger, (1969), "The combination of forecasts", Operations Research Quarterly 20, 451-468.

[5] Clements, M. y D. Hendry, (2006) "Forecasting with breaks" en Handbook of Economic Forecasting, Elliot, G., C. Granger y A. Timmermann, eds., Capítulo 12, Vol. 1, North-Holland,

[6] D 'Amato, L. y L. Garegnani (2006), "The Short -run Dynamics of Inflation:Estimating a "Hybrid New Keynesian Phillips Curve" for Argentina", Monetaria, Volumen X, CEMLA.

[7] Diebold, F.X. y R.S. Mariano, (1995), "Comparing Predicitve Accuracy", Journal of Business \& Economic Statistics 13, 253-263.

[8] -y P. Pauly, (1990), "The Use of Prior Information in Forecast Combination," International Journal of Forecasting, 6, 503-508.

[9] Elliot, G., C. Granger y A. Timmermann, eds., (2006), Handbook of Economic Forecasting, Vol. 1, North-Holland.

[10] Kendall, M. (1975), Multivariate Analysis, Charles Griffin \& Company Ltd.

[11] Hendry, D.F. y Clements, M.P., (2002), "Pooling of forecasts", Econometrics Journal 5, 1-26.

[12] Marcellino, M., (2002), "Forecasting pooling for short time series of macroeconomic variables", Oxford Bulletin of Economics and Statistics 66, 91-112. 
[13] Press, S.J., (1972), Applied Multivariate Analysis, Holt, Rinehart y Witson, Inc.un

[14] Stock, J. y M. Watson, (2006), "Forecasting with many predictors", en Handbook of Economic Forecasting, Elliot, G., C. Granger y A. Timmermann, eds., Capítulo 10, Vol. 1, North-Holland.

[15] ( $\longrightarrow$ (2005), "Why has U.S. Inflation become harder to forecast?", WP N¹2324,NBER.

[16] , (2002a), "Macroeconomic forecasting using diffusion indexes", Journal of Business and Economic Statistics 20, 147-162.

[17] —, (1999), "Forecasting Inflation", Journal of Monetary Economics,44:293-3

[18] Timmermann, A., (2006), "Forecast combinations", en Handbook of Economic Forecasting, Elliot, G., C. Granger y A. Timmermann, eds., Capítulo 10, Vol. 1, North-Holland. 


\section{Parte III}

\section{Conclusiones Generales}


Esta tesis ha investigado acerca de dos aspectos importantes de la economía argentina: los determinantes de la inestabilidad financiera y la dinámica inflacionaria. En el primer caso desarrollamos una metodología que nos permite evaluar la importancia de las tres principales hipótesis que provee la literatura para explicar el fenómeno de las crisis bancarias.

Los resultados empíricos indican que los fundamentos macroeconómicos y, en particular, el riesgo de devaluación y la caída en el nivel de actividad económica, que puede considerarse como un indicador anticipado de un deterioro en la solvencia en los bancos, juegan un papel predominante en explicar la dinámica de los depósitos durante la crisis.

Nuestros hallazgos indican que las características peculiares del régimen monetario de la Convertibilidad, que favorecía la percepción de que los deudores sería protegidos en forma permanente contra el riesgo devaluatorio, estimuló la dolarización de la cartera de los bancos, imponiendo una alto riesgo de solvencia al sistema financiero. Ese riesgo no fue percibido como tal hasta que los fundamentos macroeconómicos de la economía no dieron señales de un franco deterioro. La evidencia empírica también indica que el marco regulatorio era en ese sentido débil en relación a la exposición del sistema financiero al riesgo de devaluación y también al riesgo de la deuda pública, particularmente en un marco institucional en el que la Convertibilidad ponía fuertes restricciones al financiamiento del gobierno.

Estos hallazgos sugieren que primero, un elemento clave para permitir una profundización del sistema bancario es desarrollar instrumentos financieros atractivos en moneda local. Segundo, dado que los sistemas financieros están sujetos al riesgo devaluatorio, las regulaciones deben controlar este riesgo. Un buen diseño de regulación debe estar orientado a que los depositantes internalicen el riesgo implícito en los depósitos en moneda extranjera, pues el Banco Central no tiene instrumentos de política para actuar como un prestamista de última instancia en este caso. También parece relevante poner restricciones a los préstamos bancarios en moneda extranjera, asegurándose que los deudores en esa moneda perciban ingresos denominados en ella, de modo de que su solvencia no se vea afectada por las fluctuaciones del tipo de cambio. También la exposición al riesgo de deuda soberana de la cartera de los bancos debería estar controlada por las regulaciones.

Los capítulos II y III se han concentrado en el estudio de la dinámica inflacionaria y su relación con los distintos régimenes monetarios. En el Capítulo II nos concentramos en la persistencia inflacionaria y su dependencia del régimen monetario. Desarrollamos un ejercicio de simulación para evaluar los efectos de asumir distintos grados de presistencia de la inflación en la modelación de la política monetaria. Luego nos concentramos en el estudio de la persistencia de la inflación y su dependencia del régimen. 
Utilizamos métodos recursivos y los tests de cambio estructural desarrollados por Bai y Perron para identificar quiebres en la tasa media de inflación que resultan coincidentes con cambios en el régimen monetario: la adopción de la Convertiblidad en 1991 y el abandono de ese régimen en enero de 2002, cuando consideramos el período de baja inflación separadamente. Dada la presencia de cambios en la tasa media de inflación diferenciamos esta variable respecto de esa media que evoluciona según quiebres discretos y calculamos medidas de persistencia inflacionaria. Encontramos que la inflación fue un proceso altamente persistente durante el período de alta inflación. Con el descenso de la inflación a partir de la adopción del régimen de Convertibilidad, su persistencia se redujo marcadamente. Luego de la introducción del régimen de flotación administrada la inflación vuelve a mostar un comportamiento más persistente. Estos resultados corroboran la importancia de evaluar la presencia de quiebres estructurales al modelar la dinámica inflacionaria y en particular al intentar estimar su persistencia. También confirman que en el caso argentino la persistencia no es una característica intrínseca de la inflación, sino que depende del régimen monetario. Los resultados muestran que la persistencia inflacionaria no es muy elevada actualmente, lo que sugiere que los costos de desinflar la economía tampoco deberían serlo.

Finalmente el Capítulo III desarrolla un conjunto de modelos de pronóstico de inflación y evalúa su desempeño. Los resultados obtenidos sugieren que el desempeño relativo de los modelos causales como la curva de Phillips y los modelos monetarios versus los modelos univariados cambia dependiendo del horizonte de pronóstico. También se estudiamos la capacidad de pronóstico de modelos que utilizan como regresores medidas resumen de la variabilidad conjunta de un gran número de series económicas, usualmente denominadas factores. Los modelos univariados tienen un desempeño superior en un horizonte muy corto, pero a medida que el horizonte se extiende los modelos causales parecen aportar información relevante para el pronóstico. En particular el modelo monetario supera al modelo univariado en el horizonte de un año, lo que corrobora el rol del dinero como un determinante de largo plazo de la inflación. Finalmente los modelos basados en el uso de factores como regresores se comporta bien en los horizontes más largos, lo que sugiere que existe información relevante en los indicadores del ciclo que puede ser extraída y utilizada en el pronóstico mediante técnicas de análisis multivariado. Cuando utilizamos test estadísticos para comparar la capacidad predictiva de los modelos con el de mejor desempeño, no encontramos diferencias estadísticamente signficativas entre ellos. Es decir, no encontramos evidencia empírica que brinde argumentos contudentes para descartar ninguno de ellos a los fines de pronóstico. Por el contrario, los resultados sugieren que puede haber cierta complementariedad entre los distintos tipos de modelos, en la medida que son informativos acerca las relaciones entre la inflación y sus determinantes de corto y largo 
plazo. 